\title{
LA DESCOMPOSICIÓN DE LA VELOCIDAD EN EL PROBLEMA DE LOS N-CUERPOS Y ALGUNAS DE SUS APLICACIONES
}

\author{
Tesis que presenta \\ María Ivonne Arenas Herrera \\ Para obtener el grado de \\ Maestro en Ciencias Matemáticas
}

Asesor: Dr. Ernesto Pérez Chavela

Jurado Calificador:

Presidente: Dr. Jaime Cruz Sampedro

UAM-Azcapotzalco

Secretario: Dr. Ernesto Pérez Chavela

UAM-Iztapalapa

Vocal:

Dr. Manuel Jesús Falconi Magaña

FC-UNAM

México, D. F. Febrero 2013 


\section{En lo profesional}

* Principalmente al Dr. Ernesto Pérez Chavela, mi asesor, por dirigir esta tesis, por el interés prestado a la misma, por su tiempo y consejos. Por su apoyo en mi formación durante la maestría, y por enseñarme lo hermoso y complicado que resulta el estudio de la mecánica celeste.

* Al jurado calificador, integrado por los doctores Jaime Cruz Sampedro y Manuel Jesús Falconi Magaña, por el tiempo brindado a la revisión de esta tesis y por sus valiosas indicaciones, las cuales enriquecieron el contenido de ésta.

* A la Universidad Autónoma Metropolitana, por permitirme continuar con mi formación profesional, y a CONACyT, por la beca estímulo.

\section{En lo personal}

* A mi madre María Emma y hermanos Carlos Antoni y Pablo Fernando, por su amor y apoyo estando cerca o lejos.

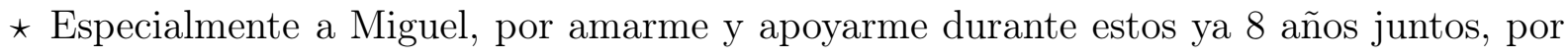
su compañía y alegría. 
1. Introducción $\quad 1$

1.1. Ecuaciones de movimiento . . . . . . . . . . . . . . . . 5

1.2. Momento de inercia . . . . . . . . . . . . . . . . . . 7

1.3. Momento angular . . . . . . . . . . . . . . . . . . 9

1.4. Integrales primeras del problema de los $N$-cuerpos . . . . . . . . . . . 12

1.5. Región de Hill . . . . . . . . . . . . . . . . . . . . . . . . . 14

1.6. Cambio configuracional . . . . . . . . . . . . . . . . 17

1.6.1. Funciones homogéneas . . . . . . . . . . . . . . 18

1.6.2. Desigualdad de Sundman y restricciones del movimiento . . . . . . . 20

1.7. Configuraciones centrales y soluciones homográficas . . . . . . . . . . . . 23

2. Descomposición de la velocidad de Saari $\quad 27$

2.1. Antecedentes . . . . . . . . . . . . . . . . . 27

2.2. Componentes de la velocidad, $W_{1}, W_{2}$ y $W_{3} \ldots \ldots \ldots$

2.3. Descomposición de la velocidad para el problema de los 2-cuerpos . . . . . . 37

3. Nueva clasificación para problemas de los $N$-cuerpos 41

3.1. Problema de los $N$-cuerpos cero-dimensional . . . . . . . . . . . . . . . 41

3.2. Problema de los $N$-cuerpos uno-dimensional . . . . . . . . . . . . . 43

3.2.1. Ejemplo 1: Problema romboidal generalizado en rotación . . . . . . . 44

3.2.2. Ejemplo 2: Problema isósceles en rotación . . . . . . . . . . . . 45

3.2.3. Ejemplo 3: Problema colineal de 3-cuerpos en el plano . . . . . . . . 46 
4. Coordenadas para obtener las componentes de la velocidad 49

4.1. Componentes de la velocidad: Problema romboidal generalizado en rotación . 49

4.2. Componentes de la velocidad: Problema isósceles en rotación . . . . . . . . . 54

4.3. Componentes de la velocidad: Problema colineal de 3-cuerpos en el plano . . 58

4.4. Nuevas coordenadas para problemas de $N$-cuerpos en el plano . . . . . . . . 62

4.5. Coordenadas de Fujiwara. . . . . . . . . . . . . . . . . . . . . . . . 62

4.5.1. Problema colineal de 3-cuerpos en coordenadas de Fujiwara . . . . . . 66

$\begin{array}{ll}\text { 5. Aplicaciones } & 69\end{array}$

5.1. Dinámica del problema romboidal generalizado en rotación . . . . . . . . . . 69

5.2. Dinámica del problema isósceles en rotación . . . . . . . . . . . . . . . 74

$\begin{array}{lr}\text { 6. Conclusiones y perspectivas } & 81\end{array}$

$\begin{array}{lr}\text { Referencias } & 87\end{array}$ 
CAPÍTULO 1

Introducción

El marco del presente trabajo es el problema de los $N$-cuerpos, el cual consiste en describir la dinámica de $N$ partículas puntuales en el espacio euclidiano $\mathbb{R}^{3}$ que se mueven bajo la ley de la gravitación universal de Isaac Newton. La mecánica celeste se encarga del estudio de los movimientos de estos cuerpos en virtud de los efectos gravitatorios que ejercen sobre ellos otros cuerpos celestes. De acuerdo a Poincaré, el objetivo final de la mecánica celeste es determinar si la mecánica newtoniana puede explicar o no, por sí sola, todos los fenómenos astronómicos, este problema en general se plantea como el problema de los $N$-cuerpos.

Las ecuaciones de movimiento para los $N$-cuerpos están dadas por la ley de la gravitación universal, la cual afirma que la fuerza de atracción que experimentan dos cuerpos dotados de masa es directamente proporcional al producto de sus masas e inversamente proporcional al cuadrado de la distancia que los separa: $F=G m_{1} m_{2} / r^{2}$, donde $F$ es la fuerza, $m_{1}$ la masa del primer cuerpo, $m_{2}$ la masa del segundo cuerpo, $r$ la distancia que los separa, y $G$ la constante de gravitación universal. Además la fuerza aplicada sobre un cuerpo, produce a éste una aceleración, dada por la fórmula $a=F / m$ ( $a$ es la aceleración, $F$ la fuerza y $m$ la masa). Es decir, la ley gravitacional de Newton y la segunda ley de Newton expresadas en forma vectorial (la fuerza y la aceleración tienen dirección), permiten escribir las ecuaciones de movimiento para el problema de los $N$-cuerpos, entonces considerando a la constante de atracción universal $G=1$ y sumando todas las fuerzas que influyen sobre la $j$-ésima masa, $m_{j}$, se obtiene que dichas ecuaciones están dadas por

$$
m_{j} \ddot{\mathbf{r}}_{j}=\mathbf{F}_{j}=\sum_{i \neq j, i=1}^{N} \frac{m_{i} m_{j}}{\left|\mathbf{r}_{i}-\mathbf{r}_{j}\right|^{3}}\left(\mathbf{r}_{i}-\mathbf{r}_{j}\right), \quad j=1, \ldots, N,
$$

donde la función $\mathbf{F}_{j}$ es la fuerza total ejercida sobre el $j$-ésimo cuerpo debido a los $N-1$ cuerpos restantes. La masa $m_{j}$ tiene posición $\mathbf{r}_{j} \in \mathbb{R}^{3}$ y aceleración $\ddot{\mathbf{r}}_{j} \in \mathbb{R}^{3}$ al tiempo $t \in \mathbb{R}$. 
Una solución al problema de los $N$-cuerpos es $\mathbf{r}(t)=\left(\mathbf{r}_{1}(t), \ldots, \mathbf{r}_{N}(t)\right) \in \mathbb{R}^{3 N}$ tal que $\mathbf{r}(t)$ satisfaga el sistema anterior. El vector fuerza lo definimos como el gradiente de una función llamada potencial ${ }^{1}$, es decir, $\mathbf{F}_{j}=\nabla U$ donde el potencial correspondiente al sistema está definido por

$$
U=\sum_{i<j} \frac{m_{j} m_{i}}{\left|\mathbf{r}_{i}-\mathbf{r}_{j}\right|}
$$

El problema más estudiado en mecánica celeste es el de 2-cuerpos, del cual se conocen las soluciones explícitas. Las ecuaciones de movimiento para el problema de los dos cuerpos son

$$
\begin{aligned}
& m_{1} \ddot{\mathbf{r}}_{1}=\frac{m_{1} m_{2}}{\left|\mathbf{r}_{2}-\mathbf{r}_{1}\right|^{3}}\left(\mathbf{r}_{2}-\mathbf{r}_{1}\right), \\
& m_{2} \ddot{\mathbf{r}}_{2}=\frac{m_{1} m_{2}}{\left|\mathbf{r}_{1}-\mathbf{r}_{2}\right|^{3}}\left(\mathbf{r}_{1}-\mathbf{r}_{2}\right),
\end{aligned}
$$

sumando ambas ecuaciones e integrando el resultado dos veces con respecto al tiempo, se tiene

$$
m_{1} \mathbf{r}_{1}+m_{2} \mathbf{r}_{2}=t \mathbf{k}+\mathbf{l}
$$

por último dividiendo la igualdad anterior entre $m_{1}+m_{2}$, se llega

$$
\frac{m_{1} \mathbf{r}_{1}+m_{2} \mathbf{r}_{2}}{m_{1}+m_{2}}=\frac{t \mathbf{k}+\mathbf{l}}{m_{1}+m_{2}}
$$

donde el miembro izquierdo es el centro de masa ${ }^{2}$ entre las dos partículas, es decir,

$$
\mathbf{r}_{c m}=\frac{m_{1} \mathbf{r}_{1}+m_{2} \mathbf{r}_{2}}{m_{1}+m_{2}}
$$

entonces el centro de masa se mueve sobre una linea recta a velocidad constante. Llevando el origen al centro de masa, es decir, para ello reemplazamos $\mathbf{r}_{1}$ por $\mathbf{r}_{1}-\mathbf{r}_{c m}$ y $\mathbf{r}_{2}$ por $\mathbf{r}_{2}-\mathbf{r}_{c m}$, el sistema (1.1) no se altera y podemos suponer que el centro de masa permanece fijo en el origen y estudiar el movimiento con respecto al centro de masa, quien se mueve uniformemente en una linea recta. Entonces fijando el centro de masa en el origen, obtenemos la siguiente relación

$$
m_{1} \mathbf{r}_{1}+m_{2} \mathbf{r}_{2}=0
$$

sustituyendo la ecuación anterior en el sistema de ecuaciones (1.1) tenemos

$$
\begin{aligned}
& \ddot{\mathbf{r}}_{1}=-\frac{m_{1}+m_{2}}{\left|\mathbf{r}_{2}-\mathbf{r}_{1}\right|^{3}} \mathbf{r}_{1}, \\
& \ddot{\mathbf{r}}_{2}=-\frac{m_{1}+m_{2}}{\left|\mathbf{r}_{1}-\mathbf{r}_{2}\right|^{3}} \mathbf{r}_{2},
\end{aligned}
$$

luego restando la primera ecuación de la segunda y haciendo $\mathbf{r}=\mathbf{r}_{2}-\mathbf{r}_{1}$, hemos reducido el sistema de los dos cuerpos a un problema clásico de fuerza central ${ }^{3}$ conocido como el problema de Kepler, es decir,

$$
\ddot{\mathbf{r}}=-\frac{\mu}{|\mathbf{r}|^{3}} \mathbf{r}
$$

\footnotetext{
${ }^{1}$ Para los físicos, el vector fuerza esta definido como el negativo del gradiente de la función potencial, esto es, $\mathbf{F}_{j}=-\nabla U$.

${ }^{2}$ Posición promedio ponderada de la masa del sistema.

${ }^{3} \mathrm{El}$ campo de fuerza depende únicamente de la distancia al origen.
} 
donde $\mu=m_{1}+m_{2}$ y $\mathbf{r} \in \mathbb{R}^{3} \backslash\{0\}$. Lo anterior nos permite decir que el movimiento de las dos partículas es equivalente al movimiento de una partícula ficticia con posición $\mathbf{r}_{2}-\mathbf{r}_{1}$, la cual se encuentra bajo la acción de la fuerza de interacción de las dos partículas. Así, el problema de los dos cuerpos y el problema de Kepler son equivalentes, en el sentido de que cualquier solución de (1.2) es una solución de (1.1), y viceversa.

Como bien se mencionó, el único caso para el cual se conocen las soluciones explícitas del problema de los $N$ cuerpos, es para el problema de $\operatorname{Kepler}(N=2)$ donde se ha demostrado que las soluciones explícitas son las cónicas ${ }^{4}$. Al aumentar el número de cuerpos la complejidad de los movimientos es mayor, de tal manera que para 3 cuerpos o más se sabe muy poco y se demostró que no es posible la integración completa, esto es, no podemos encontrar las soluciones explícitas como en el problema de Kepler. Para algunos problemas restringidos de 3 y 4 cuerpos, se han encontrado soluciones explícitas en series de potencias de las masas u otros parámetros, partiendo de la solución del problema de 2-cuerpos y considerando el efecto de los otros cuerpos como una perturbación de la solución conocida. Actualmente el problema de 3-cuerpos sigue abierto, en el sentido de encontrar las soluciones explícitas a las ecuaciones respectivas dadas cualesquiera condiciones iniciales. La importancia del problema de los $N$-cuerpos ha motivado grandes desarrollos en nuevas teorías científicas a lo largo de más de 300 años, como es el caso de la teoría cualitativa de las ecuaciones diferenciales.

Otro resultado muy importante en el problema de los $N$-cuerpos es que las únicas soluciones explícitas conocidas, son las soluciones homográficas, las cuales conservan la configuración inicial de los cuerpos salvo rotaciones y homotecias, es decir, en el problema general de los $N$-cuerpos sólo se conocen soluciones especificas que se obtienen de ciertas posiciones particulares llamadas configuraciones centrales, donde el vector de aceleración es un múltiplo escalar del vector de posición.

Las configuraciones centrales juegan un papel muy importante en el problema de los $N$ cuerpos. De hecho, se sabe que el problema de 3-cuerpos en el plano es el único donde las configuraciones centrales no dependen de la elección de las masas, y existen exactamente cinco configuraciones centrales para éste, dos en forma de triángulo equilátero (correspondientes a las dos posibles orientaciones de un triángulo en el plano) y tres colineales (correspondientes a cada una de las posibles permutaciones de las partículas). Otros ejemplos de configuraciones centrales son: cualquier $N$-polígono regular con masas iguales colocadas en cada uno de sus vértices; donde dos posibles movimientos de las partículas son contracciones o expansiones, por supuesto también puede haber rotaciones con combinación de ambos movimientos. Además también cualquier polígono regular con masas iguales colocadas en sus vértices, y una partícula de masa cualquiera, $M$, colocada en el origen siempre determina una configuración central.

La importancia y aplicación de las configuraciones centrales son muchas y variadas; por ejemplo, se sabe que la colisión total en el problema de los $N$-cuerpos es asintótica a una configuración central y que muchos escapes de partículas (escapes parabólicos) también son asintóticos a configuraciones centrales. Pero sin duda, la principal aportación de éstas, es que generan las únicas soluciones explícitas del problema de los $N$-cuerpos, las llamadas

\footnotetext{
${ }^{4}$ Todas las curvas intersección entre un cono y un plano, las cuales se clasifican en: circunferencia, elipse, parábola e hipérbola.
} 
soluciones homográficas, donde las partículas se mueven de tal forma que la configuración que forman al variar el tiempo siempre es similar a la configuración central inicial.

Debido a la dificultad para encontrar soluciones al problema de 3-cuerpos se han tratado modelos más sencillos utilizando simetrías, es decir, un camino para comprender la dinámica del problema de los $N$-cuerpos es analizar el comportamiento de sistemas particulares. Esto, por ejemplo, motiva el extenso trabajo sobre el problema isosceles de los 3-cuerpos, el problema colineal de 3-cuerpos y el problema romboidal de los 4-cuerpos.

Por la inquietud e interés que existen en continuar comprendiendo la dinámica tan complicada del problema de los $N$-cuerpos, el objetivo de este trabajo es el estudio de la descomposición de la velocidad del problema de los $N$-cuerpos, la cual fue introducida por el matemático Donald G. Saari en los años 70's. La idea de Saari de descomponer la velocidad total de un sistema de $N$-partículas moviéndose bajo sus atracciones gravitacionales fue motivada por la necesidad de encontrar nuevas herramientas para entender mejor la dinámica tan complicada del problema de los $N$-cuerpos en general y la de los tres cuerpos en particular, en especial en el estudio de los movimientos acotados.

Como objetivos particulares en este trabajo se tienen, dar una nueva forma de clasificar el problema clásico de los $N$-cuerpos según la complejidad de la dinámica que presenten, relacionar las constantes de movimiento, integral de energía total y momento angular con la intención de analizar aspectos cualitativos relacionados con el escape de las partículas, y hacer una generalización de las regiones de Hill. En general, el propósito es utilizar algunos de los conceptos ya conocidos del problema de los $N$-cuerpos y junto con la descomposición de la velocidad, aplicarlo a problemas de los $N$-cuerpos para encontrar una relación entre las constantes de movimiento, integral total y momento angular, y así poder estudiar los movimientos que se producen en ciertos problemas particulares.

En el capítulo 1, planteamos las ecuaciones de movimiento del problema de los $N$-cuerpos y estudiamos algunos aspectos generales tales como el momento de inercia y el momento angular, de éste último estudiamos su comportamiento cuando las partículas están restringidas a rotar en un plano. Se estudia también cómo encontrar las integrales primeras del problema de los $N$-cuerpos cuando las partículas se mueven en el espacio. También en este capítulo se estudian las regiones de Hill e interpretamos su significado con dos ejemplos tales como el problema de Kepler en el plano y el problema isósceles de los 3-cuerpos. Explicamos lo que en este trabajo se entenderá por cambio configuracional, definimos las funciones homogéneas y estudiamos algunas de sus propiedades las cuales aplicamos a las funciones potencial y momento de inercia, para obtener una función que determina ciertos cambios en la forma de las configuraciones. Después se deduce la desigualdad de Sundman y con ayuda de ésta describimos restricciones en el movimiento posible de las partículas, las cuales son válidas para problemas de los $N$-cuerpos. Por último en este capítulo también se estudia la existencia de soluciones especiales generadas por configuraciones centrales, y se demuestra que una configuración central es un punto crítico de la función que determina ciertos cambios en la forma de la configuración.

En el capítulo 2, iniciamos el estudio de la descomposición de la velocidad, planteando la idea geométrica de la cual partió Saari, luego definimos un producto escalar y producto vectorial para el sistema de los $N$-cuerpos; y hacemos ver cómo éstos simplifican la notación de 
las primeras integrales de movimiento y el momento de inercia. Posteriormente presentamos las tres componentes que conforman la descomposición de la velocidad de Saari, las cuales son: $W_{1}$ : velocidad rotacional, $W_{2}$ : velocidad escalar y $W_{3}$ : velocidad configuracional; dedicadas a las rotaciones del sistema vistas como un cuerpo rígido, al cambio en el tamaño del sistema y al cambio en la forma de la configuración, respectivamente. Estudiamos a detalle el significado y comportamiento de cada una de esas tres componentes. Por último, obtenemos la descomposición de la velocidad para el problema de los dos cuerpos, y analizamos el comportamiento de cada una de las componentes del vector de velocidad del sistema.

En el capítulo 3, estudiamos una nueva forma de clasificar problemas de los $N$-cuerpos, es decir, obtenemos una clasificación en términos de la descomposición de la velocidad de Saari, introducimos dos definiciones que permiten iniciar con dicha clasificación: sistemas cero-dimensional y sistemas uno-dimensional. También estudiamos tres ejemplos de sistemas uno-dimensional: el problema romboidal generalizado en rotación, el problema isósceles en rotación y el problema colineal de tres cuerpos en el plano.

En el capítulo 4, se obtienen diferentes sistemas coordenados para analizar con más profundidad las tres componentes de la velocidad de cada uno de los sistemas uno-dimensionales estudiados en el capítulo anterior. También se estudiarán nuevas coordenadas llamadas coordenadas de Fujiwara, las cuales permitirán definir un nuevo sistema de coordenadas para problemas de los $N$-cuerpos en el plano, observaremos que éstas describen el cambio en la forma de la configuración dada por las variables originales, y probaremos algunos resultados relacionados. Estudiaremos la forma de las tres componentes de la velocidad en coordenadas de Fujiwara. Por último, como una aplicación de dichas coordenadas escribiremos el problema colineal de los tres cuerpos en el plano en coordenadas de Fujiwara, considerando dos casos: en el primero cuando las partículas presentan movimiento de rotación, y el segundo cuando las partículas no presentan movimiento de rotación.

En el capítulo 5, estudiaremos lo que en física es conocido como el potencial aumentado, esto es, el potencial clásico más la acción del momento angular, lo cual coincidirá exactamente con la primer componente de nuestra descomposición. Como una aplicación importante de la descomposición de la velocidad encontraremos una relación entre las constantes de movimiento, integral de energía total y momento angular con el escape de partículas, en particular obtendremos las condiciones necesarias para poder tener escapes en los problemas romboidal generalizado en rotación e isósceles en rotación. Lograremos una generalización de las regiones de Hill.

Finalmente en el capítulo 6 se establecen las conclusiones y perspectivas.

\subsection{Ecuaciones de movimiento}

Consideremos el problema de los $N$-cuerpos, el cual consiste en describir el movimiento de $N$ masas puntuales moviéndose bajo la ley de atracción de Newton, que como sabemos nos dice que la atracción entre dos partículas es directamente proporcional al producto de sus masas e inversamente proporcional al cuadrado de la distancia que las separa. Si suponemos que la $j$-ésima partícula tiene masa positiva, es decir, $m_{j} \in \mathbb{R}^{+}$y al tiempo $t$ está en la posición 
espacial $\mathbf{r}_{j}=\left(x_{j}, y_{j}, z_{j}\right)$, el movimiento de cada partícula está descrito por

$$
m_{j} \ddot{\mathbf{r}}=\mathbf{F}_{j}, \quad j=1, \ldots, N
$$

donde $\mathbf{F}_{j}$ es la fuerza gravitacional resultante que actúa sobre la partícula $j$. Considerando a la constante de atracción universal $G=1$ y sumando todas las fuerzas que influyen sobre $m_{j}$, se obtiene que las ecuaciones del problema de $N$-cuerpos están dadas por

$$
m_{j} \ddot{\mathbf{r}}_{j}=\mathbf{F}_{j}=\sum_{i \neq j, i=1}^{N} \frac{m_{i} m_{j}}{\left|\mathbf{r}_{i}-\mathbf{r}_{j}\right|^{3}}\left(\mathbf{r}_{i}-\mathbf{r}_{j}\right), \quad j=1, \ldots, N .
$$

Sea $\mathbf{r}=\left(\mathbf{r}_{1}, \ldots, \mathbf{r}_{N}\right)^{t} \in \mathbb{R}^{3 N}$ el vector de posición de las partículas, el cual llamamos configuración del sistema de partículas, es decir, este vector describe las posibles posiciones del sistema de $N$-partículas; y sea $X$ el espacio de configuraciones

$$
X=\left\{\mathbf{r}=\left(\mathbf{r}_{1}, \ldots, \mathbf{r}_{N}\right)^{t} \in \mathbb{R}^{3 N} \mid \mathbf{r}_{i} \neq \mathbf{r}_{j}, \forall i \neq j\right\} .
$$

Es claro, que el sistema (1.3) no está definido cuando dos o más partículas están en una misma posición, es decir, cuando hay colisión de dos o más cuerpos, para aclarar esto definamos

$$
\Delta_{i j}=\left\{\left(\mathbf{r}_{1}, \ldots, \mathbf{r}_{N}\right) \in \mathbb{R}^{3 N} \mid \mathbf{r}_{i}=\mathbf{r}_{j}\right\} \quad \text { y } \quad \Delta=\bigcup_{i<j} \Delta_{i j}
$$

Por lo tanto, podemos escribir el espacio de configuraciones como $X=\mathbb{R}^{3 N} \backslash \Delta$. La fuerza newtoniana se puede expresar como la derivada de la energía potencial,

$$
U: X \rightarrow \mathbb{R}^{+}, \quad U(\mathbf{r})=\sum_{i<j} \frac{m_{j} m_{i}}{\left|\mathbf{r}_{i}-\mathbf{r}_{j}\right|}
$$

donde $\mathbf{r}_{j}$ es la posición de cada partícula en $\mathbb{R}^{3}$ y $m_{j}$ su masa.

Es decir, el campo vectorial (1.3) resulta ser el gradiente de la función potencial dada por la ecuación (1.4), con lo cual el sistema de ecuaciones (1.3) se puede expresar de las dos formas siguientes

$$
\begin{aligned}
m_{j} \ddot{\mathbf{r}}_{j} & =\nabla_{j} U(\mathbf{r})=\frac{\partial U}{\partial \mathbf{r}_{j}}, \quad j=1, \ldots, N, \\
M \ddot{\mathbf{r}} & =\nabla U(\mathbf{r}),
\end{aligned}
$$

donde

$$
\nabla_{j}=\left(\frac{\partial}{\partial x_{j}}, \frac{\partial}{\partial y_{j}}, \frac{\partial}{\partial z_{j}}\right)^{t}, \nabla=\left(\nabla_{1}, \ldots, \nabla_{N}\right)^{t}
$$

y $M=\operatorname{diag}\left(m_{1}, m_{1}, m_{1}, \ldots, m_{N}, m_{N}, m_{N}\right)$ es la matriz diagonal cuadrada de tamaño $3 N$, cuyas entradas corresponden a las masas de las partículas involucradas.

Sean $\mathbf{p}_{j}=m_{j} \dot{\mathbf{r}}_{j}, \mathbf{p}=M \dot{\mathbf{r}}$, llamados momento lineal de la partícula $j$ y momento lineal del sistema de partículas, respectivamente. Entonces el problema de los $N$-cuerpos se escribe como un sistema de $6 N$ ecuaciones diferenciales de primer orden

$$
\begin{aligned}
\dot{\mathbf{r}} & =M^{-1} \mathbf{p}, \\
\dot{\mathbf{p}} & =\nabla U(\mathbf{r}) .
\end{aligned}
$$


El dominio de definición del sistema (1.5), es el espacio fase, es decir, dado $\mathbf{r}$ en $\mathbb{R}^{3 N} \backslash \Delta$ identificamos su espacio tangente con $\mathbb{R}^{3 N}$. Entonces el espacio fase del sistema (1.5) es

$$
T X=\left(\mathbb{R}^{3 N} \backslash \Delta\right) \times T \mathbb{R}^{3 N}=\left\{(\mathbf{r}, \mathbf{p}) \mid \mathbf{r} \in X, \mathbf{p} \in T_{\mathbf{r}} X\right\},
$$

donde $T \mathbb{R}^{3 N}$ es el haz tangente ${ }^{5}$ de $\mathbb{R}^{3 N}$. Una trayectoria u órbita en $T X$, es una solución $(\mathbf{r}(t), \mathbf{p}(t))$ del sistema de ecuaciones (1.5), definida en su intervalo maximal, es decir, para cada punto $(\mathbf{r}(t), \mathbf{p}(t)) \in T X$, la teoría de ecuaciones diferenciales nos dice que por este punto pasa una única solución definida en un intervalo maximal, la cual es analítica.

En este trabajo estamos interesados en el estudio de soluciones acotadas, razón por la cual en el siguiente apartado nos enfocaremos en estudiar el momento de inercia, el cual es importante en el desarrollo de este trabajo.

\subsection{Momento de inercia}

En mecánica celeste el momento de inercia es una forma cuadrática positiva definida, la cual establece una forma de medir el tamaño del sistema, y se define a continuación.

Definición 1.1 Dado un sistema de $N$-partículas, el momento de inercia del mismo se define como la suma de los productos de las masas de las partículas por el cuadrado de la distancia al origen de cada partícula. Es decir, matemáticamente se expresa como

$$
I=\sum_{j=1}^{N} m_{j}\left|\mathbf{r}_{j}\right|^{2} .
$$

Si ahora hacemos $\left|\mathbf{r}_{j}\right|=x_{j}$ y $a_{j}=\sqrt{\frac{1}{m_{j}}}$ (el cual está bien definido puesto que $m_{j}>0$ para todo $j=1, \ldots, N)$, entonces el momento de inercia toma la forma

$$
I=\sum_{j=1}^{N} \frac{x_{j}^{2}}{a_{j}^{2}},
$$

observemos que cuando $I=1$, la ecuación anterior claramente representa un elipsoide en $\mathbb{R}^{N}$ que corta los ejes coordenados, $\mathbf{e}_{j}$, en $+a_{j} \mathrm{y}-a_{j}$. Dicho elipsoide es conocido en mecánica celeste como el elipsoide de masas, y se representa por

$$
\left\{\mathbf{r} \in X \mid I(\mathbf{r})=\mathbf{r}^{t} M \mathbf{r}=1\right\} .
$$

Obviamente cuando consideramos todas las masas iguales, es decir, $m_{j}=m$ para todo $j=1, \ldots, N$, obtenemos una esfera en $\mathbb{R}^{N}$ de radio $r=\sqrt{\frac{1}{m}}$. Entonces $\sqrt{I}$ es el radio del

\footnotetext{
${ }^{5}$ Sea $M$ una variedad diferenciable cerrada. Sea $p \in M$ y sea $T_{p} M$ el espacio tangente a $M$ en $p$. El haz tangente $T M$ de $M$ es una variedad diferenciable dada por $T M=\bigcup_{p \in M} T_{p} M$.
} 
elipsoide de masas del sistema de los $N$-cuerpos, por lo cual el momento de inercia establece una forma de medir el tamaño del sistema. La interpretación geométrica del elipsoide de masas para el caso $N=3$ se observa en la figura 1.1 .

Por otro lado, existe una forma alternativa de escribir el momento de inercia, $I$, la cual es válida para el caso de tener el centro de masa fijo al origen, y está dada en la siguiente proposición.

Proposición 1.2 El momento de inercia I puede ser expresado como

$$
I=\frac{1}{\widetilde{m}} \sum_{j<k} m_{j} m_{k}\left|\mathbf{r}_{j}-\mathbf{r}_{k}\right|^{2}
$$

donde $\widetilde{m}=m_{1}+m_{2}+\ldots+m_{N}$ es la masa total del sistema.

Demostración:

Por expansión,

$$
\begin{aligned}
\sum_{j=1}^{N} \sum_{k=1}^{N} m_{j} m_{k}\left|\mathbf{r}_{j}-\mathbf{r}_{k}\right|^{2} & =2 \sum_{k=2}^{N} \sum_{j=1}^{k-1} m_{j} m_{k}\left|\mathbf{r}_{j}-\mathbf{r}_{k}\right|^{2} \\
& =2 \sum_{j<k} m_{j} m_{k}\left|\mathbf{r}_{j}-\mathbf{r}_{k}\right|^{2}
\end{aligned}
$$

Sustituyendo lo anterior en la expresión (1.7) y desarrollando el módulo al cuadrado se obtiene

$$
\begin{aligned}
\frac{1}{\widetilde{m}} \sum_{j<k} m_{j} m_{k}\left|\mathbf{r}_{j}-\mathbf{r}_{k}\right|^{2} & =\frac{1}{2 \widetilde{m}} \sum_{j=1}^{N} \sum_{k=1}^{N} m_{j} m_{k}\left|\mathbf{r}_{j}-\mathbf{r}_{k}\right|^{2} \\
& =\frac{1}{2 \widetilde{m}} \sum_{j=1}^{N} \sum_{k=1}^{N} m_{j} m_{k}\left[\left|\mathbf{r}_{j}\right|^{2}+\left|\mathbf{r}_{k}\right|^{2}-2 \mathbf{r}_{j} \cdot \mathbf{r}_{k}\right]
\end{aligned}
$$

Pero

Luego

$$
\sum_{k=1}^{N} \sum_{j=1}^{N} m_{j} m_{k}\left|\mathbf{r}_{j}\right|^{2}=\sum_{k=1}^{N} m_{k} \sum_{j=1}^{N} m_{j}\left|\mathbf{r}_{j}\right|^{2}=\widetilde{m} I
$$

$$
\sum_{k=1}^{N} \sum_{j=1}^{N} m_{j} m_{k}\left|\mathbf{r}_{k}\right|^{2}=\sum_{j=1}^{N} m_{j} \sum_{k=1}^{N} m_{k}\left|\mathbf{r}_{k}\right|^{2}=\widetilde{m} I .
$$

Para el término restante, se tiene por la integral de centro de masa

$$
-2 \sum_{j=1}^{N} \sum_{k=1}^{N} m_{k} m_{j} \mathbf{r}_{j} \cdot \mathbf{r}_{k}=-2 \sum_{j=1}^{N} \sum_{k=1}^{N} m_{j} \mathbf{r}_{j} \cdot m_{k} \mathbf{r}_{k}=-2 \sum_{j \neq 1}^{N} m_{j} \mathbf{r}_{j} \cdot \sum_{k \neq 1}^{N} m_{k} \mathbf{r}_{k}=0 .
$$

Por lo tanto sustituyendo los tres resultados anteriores en la última igualdad de la ecuación (1.8), se obtiene lo que se quiere probar, esto es

$$
\frac{1}{\widetilde{m}} \sum_{j<k} m_{j} m_{k}\left|\mathbf{r}_{j}-\mathbf{r}_{k}\right|^{2}=\frac{1}{2 \widetilde{m}}(\widetilde{m} I+\widetilde{m} I)=I .
$$




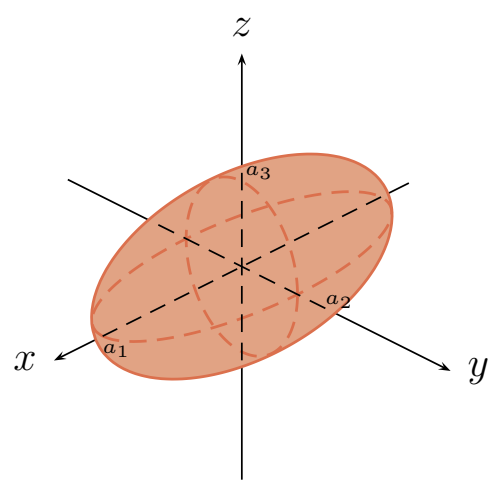

(a) Tres masas diferentes generan un Elipsoide de Masas en $\mathbb{R}^{3}$ que corta a los ejes en $a_{j}= \pm \sqrt{\frac{1}{m_{j}}} \operatorname{con} j=1,2,3$.

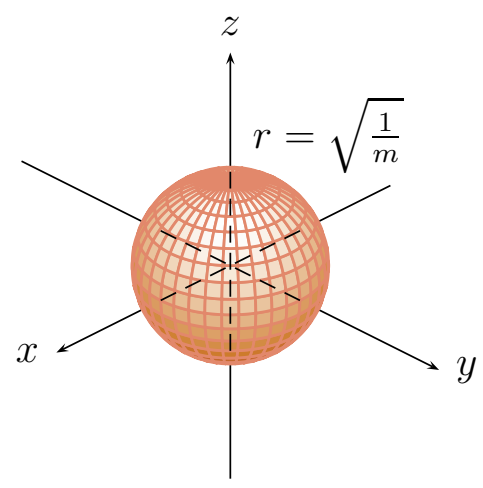

(b) Tres masas iguales generan una esfera en $\mathbb{R}^{3}$ de radio $r=\sqrt{\frac{1}{m}}$.

Figura 1.1: Elipsoide de masas para $N=3$.

La proposición 1.2 nos dice que el momento de inercia se puede escribir en términos de las distancias mutuas entre las partículas, y de igual forma describe el tamaño del sistema, puesto que una colisión total ocurre si y sólo si $I=0$.

Por lo tanto, la forma natural de medir los movimientos acotados es este viejo concepto de la física conocido como momento de inercia.

\subsection{Momento angular}

En el estudio del problema de los $N$-cuerpos son de gran importancia los movimientos de rotación, en los cuales se aplican los principios de la mecánica de Newton, y adquieren especial relevancia magnitudes físicas como el momento angular, que es característico de cada cuerpo en rotación. Se llama rotación al movimiento de un cuerpo con respecto a un eje de giro interior o externo al mismo.

En el estudio de las rotaciones el momento angular es una magnitud fundamental, esta magnitud desempeña respecto a las rotaciones un papel análogo al momento lineal en las traslaciones. El momento angular para un cuerpo rígido que rota respecto a un eje, es la resistencia que ofrece dicho cuerpo a la variación de la velocidad angular. En mecánica newtoniana, el momento angular de una partícula o masa puntual con respecto a un punto $O$ del espacio, se define como el momento de su cantidad de movimiento con respecto a ese punto.

Consideremos una partícula de masa $m$ cuya posición (respecto a algún sistema de referencia inercial) viene dada por el vector $\mathbf{r}$. Sea $\mathbf{F}$ la fuerza neta que actúa sobre dicha 
partícula. Entonces, de acuerdo a la segunda ley de Newton, la ecuación de movimiento es

$$
\mathbf{F}=\frac{d}{d t} m \mathbf{v}=\frac{d \mathbf{p}}{d t}
$$

Tomando el producto cruz con el vector $\mathbf{r}$ se obtiene

$$
\mathbf{r} \times \mathbf{F}=\mathbf{r} \times \frac{d \mathbf{p}}{d t}
$$

Observemos que

$$
\frac{d}{d t}(\mathbf{r} \times \mathbf{p})=\frac{d \mathbf{r}}{d t} \times \mathbf{p}+\mathbf{r} \times \frac{d \mathbf{p}}{d t}=\mathbf{r} \times \frac{d \mathbf{p}}{d t} .
$$

La última igualdad se deduce del hecho que los vectores $\frac{d \mathbf{r}}{d t}=\mathbf{v}$ y $\mathbf{p}$ son paralelos. Usando (1.9) en (1.10) se obtiene

$$
\vec{\tau}=\mathbf{r} \times \mathbf{F}=\frac{d}{d t}(\mathbf{r} \times \mathbf{p}) .
$$

Definimos el momento angular de una partícula por

$$
\mathbf{c}=\mathbf{r} \times m \mathbf{v},
$$

entonces

$$
\vec{\tau}=\frac{d \mathbf{c}}{d t}
$$

La interpretación geométrica del momento angular de una partícula se ve en la figura 1.2. El momento angular de una partícula depende del origen que se use para evaluarlo, entonces el momento angular de la partícula respecto al mismo origen, no variará en el tiempo, es decir, se conservará.

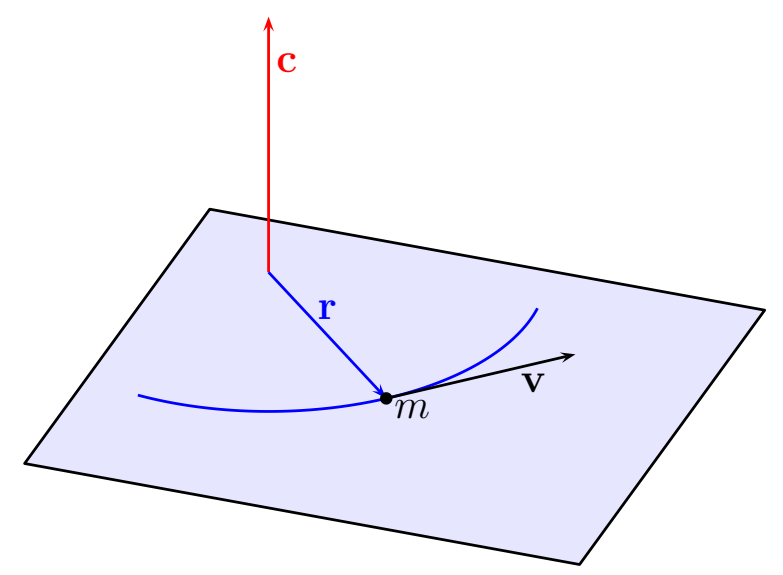

Figura 1.2: Momento angular de una partícula.

Ahora estudiaremos el momento angular de una partícula moviéndose en el plano, esto es, la partícula esta restringida a tener movimiento de rotación en un plano. Sin pérdida de 
generalidad supongamos que dicha partícula de masa $m$ se mueve en el plano $x-y$, y sean $r(t), \theta(t)$ las coordenadas polares de su vector de posición $\mathbf{r}(t)$. Entonces la posición de la partícula vendrá dada por

$$
\mathbf{r}=r(\cos \theta, \operatorname{sen} \theta, 0)=r(\cos \theta \hat{x}+\operatorname{sen} \theta \hat{y})=r \hat{r},
$$

donde $\hat{r}=\cos \theta \hat{x}+\operatorname{sen} \theta \hat{y}$ y con $\hat{x}=(1,0,0), \hat{y}=(0,1,0)$ vectores unitarios del espacio $\mathbb{R}^{3}$.

Luego, derivando $\mathbf{r}$ obtenemos la velocidad

$$
\mathbf{v}=\dot{r} \hat{r}+r \dot{\hat{r}}
$$

Pero

$$
\dot{\hat{r}}=\frac{d}{d t}(\cos \theta \hat{x}+\operatorname{sen} \theta \hat{y})=\dot{\theta}(-\operatorname{sen} \theta \hat{x}+\cos \theta \hat{y})=\dot{\theta} \hat{\theta},
$$

donde $\hat{\theta}=-\operatorname{sen} \theta \hat{x}+\cos \theta \hat{y}$, de aquí se tiene que

$$
\mathbf{v}=\dot{r} \hat{r}+r \dot{\theta} \hat{\theta}
$$

De esta manera utilizando propiedades del producto vectorial encontramos para el momento angular de la partícula en el plano, la siguiente expresión

$$
\mathbf{c}=\mathbf{r} \times \mathbf{p}=m r \hat{r} \times \mathbf{v}=m r \dot{r} \hat{r} \times \hat{r}+m r^{2} \dot{\theta} \hat{r} \times \hat{\theta}=m r^{2} \dot{\theta} \hat{z},
$$

donde $\hat{r} \times \hat{\theta}=(\cos \theta, \operatorname{sen} \theta, 0) \times(-\operatorname{sen} \theta, \cos \theta, 0)=(0,0,1)=\hat{z}$.

Hemos encontrado que el vector momento angular para esta partícula situada en el plano $x-y$ tiene la dirección del eje $z$, en general, el vector momento angular no tiene la dirección del eje de rotación, es decir, el vector momento angular no coincide con su proyección a lo largo del eje de rotación, pero observemos que el último término de la ecuación (1.11) indica que el vector momento angular coincide con el eje de rotación que en este caso es el eje $z$, para estos ejes existe una relación sencilla entre el momento de inercia y la velocidad angular, puesto que, el cuadrado de la distancia al origen de la partícula $\mathbf{r}$ es

$$
|\mathbf{r}|^{2}=r^{2}\left(\cos ^{2} \theta+\operatorname{sen}^{2} \theta\right)=r^{2},
$$

de aquí en la última igualdad de la ecuación (1.11) el término $m r^{2}=m|\mathbf{r}|^{2}=I$ (momento de inercia de una partícula), así podemos escribir la ecuación (1.11) como

$$
\mathbf{c}=I \dot{\theta} \hat{z} .
$$

Por lo tanto, el momento angular de una partícula de masa $m$ que se mueve en el plano $x-y$, resulta ser el producto de su momento de inercia y velocidad angular.

Por otro lado, el momento angular para un sistema de $N$-partículas se define de la siguiente manera. 
Definición 1.3 Para un sistema de $N$-partículas, y con respecto a un origen de referencia, el momento angular total se define como la suma de los momentos angulares de cada partícula para dicho punto. Es decir,

$$
\mathbf{c}=\sum_{j=1}^{N} m_{j} \mathbf{r}_{j} \times \mathbf{v}_{j}
$$

siendo $\mathbf{r}_{j}$ el vector de posición de cada partícula, $m_{j}$ su masa $y \mathbf{v}_{j}$ su velocidad.

Por último, para un sistema de $N$-partículas moviéndose en el plano $x$ - $y$, de igual forma se cumple que su momento angular está relacionado con el momento de inercia del sistema y la velocidad angular del mismo.

\subsection{Integrales primeras del problema de los $N$-cuerpos}

Una integral primera del sistema de ecuaciones (1.5), es una función $H: T X \rightarrow \mathbb{R}$ continua, tal que $H(\mathbf{r}, \mathbf{p})$ permanece constante a lo largo de cada órbita del espacio fase $T X$.

A continuación describimos las diez integrales primeras que se conocen para el problema de los $N$-cuerpos, cuando las partículas se mueven en el espacio.

\section{Integrales del momento lineal}

Para una solución cualquiera $(\mathbf{r}, \mathbf{p})$ del sistema (1.5), se tiene que

$$
\sum_{j=1}^{N} \dot{\mathbf{p}}_{j}=\sum_{j=1}^{N} \nabla_{j} U(\mathbf{r})=\sum_{j=1}^{N} \sum_{i \neq j, i=1}^{N} \frac{m_{i} m_{j}}{\left|\mathbf{r}_{i}-\mathbf{r}_{j}\right|^{3}}\left(\mathbf{r}_{i}-\mathbf{r}_{j}\right)=\mathbf{0}
$$

donde en la última sumatoria los términos se anulan por parejas. Entonces $\frac{d}{d t} \sum \mathbf{p}_{j}(t)=\mathbf{0}$, lo cual implica, que $\sum \mathbf{p}_{j}(t)$ es constante sobre toda solución,

$$
\sum_{j=1}^{N} \mathbf{p}_{j}(t)=\mathbf{a}, \quad \mathbf{a} \in \mathbb{R}^{3} \quad \text { constante. }
$$

En este caso decimos que la solución tiene momento lineal a. De aquí obtenemos tres integrales primeras, conocidas como integrales de momento lineal

$$
H_{m l}(\mathbf{p})=\sum_{j=1}^{N} \mathbf{p}_{j}
$$




\section{Integrales del centro de masa}

La ecuación (1.13) implica que $\frac{d}{d t} \sum m_{j} \mathbf{r}_{j}(t)=\mathbf{a}$, en todos los puntos de la solución $(\mathbf{r}(t), \mathbf{p}(t))$. Entonces,

$$
\sum_{j=1}^{N} m_{j} \mathbf{r}_{j}(t)=\mathbf{a} t+\mathbf{b}, \quad \mathbf{a}, \mathbf{b} \in \mathbb{R}^{3} \quad \text { constantes. }
$$

Es decir, $\mathbf{c}_{m}=\frac{\sum m_{j} \mathbf{r}_{j}}{\sum m_{j}}$ el centro de masa del sistema de partículas, tiene movimiento rectilineo uniforme. Entonces dada una solución $(\mathbf{r}(t), \mathbf{p}(t))$ con momento lineal a, obtenemos la siguiente solución con momento lineal cero y centro de masa fijo en el origen,

$$
\bar{r}_{j}(t)=\mathbf{r}_{j}(t)-\mathbf{c}_{m}(t), \quad \bar{p}_{j}(t)=\mathbf{p}_{j}(t)-\frac{m_{j}}{\sum m_{j}} \mathbf{a},
$$

y para las cuales la siguiente función vectorial representa tres nuevas integrales primeras, conocidas como integrales del centro de masa

$$
H_{c m}(\mathbf{r})=\sum_{j=1}^{N} m_{j} \mathbf{r}_{j}
$$

\section{Integrales de momento angular}

Para una solución cualquiera $(\mathbf{r}(t), \mathbf{p}(t))$, se tiene que

$$
\sum_{j=1}^{N} \mathbf{r}_{j} \times m_{j} \ddot{\mathbf{r}}=\sum_{j=1}^{N} \mathbf{r}_{j} \times \sum_{i \neq j, i=1}^{N} \frac{m_{i} m_{j}}{\left|\mathbf{r}_{i}-\mathbf{r}_{j}\right|^{3}}\left(\mathbf{r}_{i}-\mathbf{r}_{j}\right)=\sum_{j=1}^{N} \sum_{i \neq j, i=1}^{N} \frac{m_{i} m_{j}}{\left|\mathbf{r}_{i}-\mathbf{r}_{j}\right|^{3}}\left(\mathbf{r}_{j} \times \mathbf{r}_{i}\right)=\mathbf{0},
$$

donde los términos de la última sumatoria se anulan por parejas. Entonces $\frac{d}{d t} \sum \mathbf{r}_{j} \times \mathbf{p}_{j}=\mathbf{0}$, lo cual implica que

$$
\sum_{j=1}^{N} \mathbf{r}_{j} \times \mathbf{p}_{j}=\mathbf{c}, \quad \mathbf{c} \in \mathbb{R}^{3} \quad \text { constante. }
$$

En este caso decimos que la solución tiene momento angular c. De la relación anterior, obtenemos otras tres integrales primeras conocidas como integrales del momento angular

$$
H_{m a}(\mathbf{r}, \mathbf{p})=\sum_{j=1}^{N} \mathbf{r}_{j} \times \mathbf{p}_{j}
$$

\section{Integral de energía}

Definimos la energía cinética del sistema de partículas, $T=\frac{1}{2} \sum_{j=1}^{N} m_{j}\left|\mathbf{v}_{j}\right|^{2}$, como

$$
T: \mathbb{R}^{3 N} \rightarrow \mathbb{R}^{+}, \quad T(\mathbf{p})=\frac{1}{2} \mathbf{p}^{t} M^{-1} \mathbf{p}=\frac{1}{2} \sum_{j=1}^{N} m_{j}^{-1}\left|\mathbf{p}_{j}\right|^{2}
$$


Sobre una solución cualquiera $(\mathbf{r}(t), \mathbf{p}(t))$, se cumple que

$$
\begin{aligned}
\dot{T} & =\sum_{j=1}^{N} m_{j}^{-1} \mathbf{p}_{j} \cdot \dot{\mathbf{p}}_{j}=\sum_{j=1}^{N} m_{j} \dot{\mathbf{r}}_{j} \cdot \ddot{\mathbf{r}}_{j}=\widetilde{m} \dot{\mathbf{r}} \cdot \ddot{\mathbf{r}}, \\
\dot{U} & =\sum_{j=1}^{N} \frac{\partial U}{\partial \mathbf{r}_{j}} \dot{\mathbf{r}_{j}}=\nabla U \cdot \dot{\mathbf{r}}=\tilde{m} \ddot{\mathbf{r}} \cdot \dot{\mathbf{r}}
\end{aligned}
$$

entonces $\frac{d}{d t}(T-U)=0$, lo cual implica que $T-U$ es constante sobre toda la solución,

$$
T(\mathbf{p}(t))-U(\mathbf{r}(t))=h, \quad h \in \mathbb{R},
$$

y decimos que la solución tiene energía $h$, la relación anterior nos da otra integral primera llamada integral de energía

$$
H(\mathbf{r}, \mathbf{p})=T(\mathbf{p})-U(\mathbf{r}) .
$$

Conocemos que para el problema de los 2-cuerpos las soluciones explícitas están dadas por las cónicas, y que para el problema de los $N$-cuerpos (con $N \geq 3$ ) no podemos encontrar las soluciones explícitas como en el problema de los dos cuerpos, por éste hecho es que tantos matemáticos y astrónomos eminentes se han dedicado a estudiarlo a lo largo de más de 300 años, habiéndose expuesto en consecuencia, varios trabajos, todos ellos condicionados por las anteriores diez integrales primeras del movimiento que se conocen para el problema de los $N$-cuerpos.

\subsection{Región de Hill}

El Hamiltoniano es la función dada por la ecuación (1.14), siendo que $H$ es una integral primera, entonces la energía total del sistema es una constante de movimiento que podemos escribir como

$$
h=T(\mathbf{p})-U(\mathbf{r}),
$$

y dado que la energía cinética siempre cumple $T \geq 0$, entonces el Hamiltoniano está definido para cada valor de la energía $h$, así, la región determinada por el conjunto

$$
\left\{\mathbf{r} \in \mathbb{R}^{n} \mid h \geq-U(\mathbf{r})\right\},
$$

es llamada la región de Hill. Esta región determina el tipo de movimientos en el espacio de configuraciones, es decir, clasifica las soluciones en acotadas o no acotadas según la distancia entre las partículas y el nivel de energía en el que nos encontremos. En realidad, lo que estamos haciendo es proyectar el espacio fase al espacio de configuraciones.

Al subconjunto de la región de Hill cuyos elementos satisfacen

$$
h=-U(\mathbf{r}),
$$

se le conoce como el conjunto de velocidad cero, ya que en esta posición la energía cinética es nula. Con el propósito de resaltar la gran utilidad de las regiones de Hill, abordaremos 
dos ejemplos conocidos del problema de los $N$-cuerpos, tales como el problema de Kepler en el plano y el problema isósceles de los 3-cuerpos, encontraremos sus respectivas regiones de Hill y con ellas conoceremos en qué casos las partículas presentan movimientos acotados o no acotados.

\section{Problema de Kepler en el plano}

El problema de los 2-cuerpos puede reducirse a un problema de fuerza central y hamiltoniano dado por

$$
H(\mathbf{r}, \mathbf{p})=\frac{1}{2}|\mathbf{p}|^{2}-\frac{1}{|\mathbf{r}|},
$$

entonces la región de Hill está dada por el conjunto

$$
\left\{\mathbf{r} \in \mathbb{R}^{2} \mid h \geq-\frac{1}{\sqrt{r_{1}^{2}+r_{2}^{2}}}\right\} .
$$

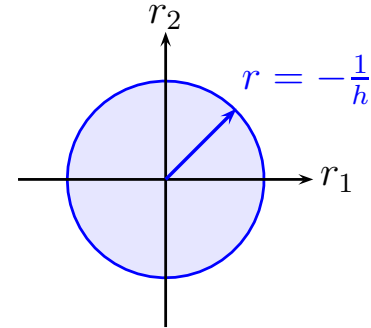

(a) Si $h<0$ entonces la región de Hill es $r_{1}^{2}+r_{2}^{2} \leq \frac{1}{h^{2}}$.

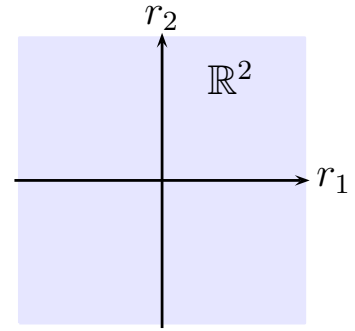

(b) Si $h \geq 0$ entonces la región de Hill es todo $\mathbb{R}^{2}$.

Figura 1.3: Regiones de Hill en el problema de Kepler en el plano.

Tomando niveles de energía negativos, $h<0$, se tiene la siguiente desigualdad

$$
r_{1}^{2}+r_{2}^{2} \leq \frac{1}{h^{2}}
$$

entonces el movimiento de las partículas cuando $h<0$ es acotado, puesto que las masas se mueven dentro o sobre el círculo de radio $r=-\frac{1}{h}$, como lo hace ver en la figura $1.3(a)$. Si consideramos niveles de energía $h \geq 0$, sucede lo contrario ya que las partículas tienen la libertad de moverse sobre todo $\mathbb{R}^{2}$, es decir, presentan movimiento no acotado. La figura 1.3 (b) muestra la situación.

\section{Problema isósceles de los 3-cuerpos}

Consideremos tres partículas en el plano que se mueven de tal suerte que en todo momento se mantiene la configuración de un triángulo isósceles. Dos de ellas tienen masas iguales que suponemos iguales a 1 , y la tercera tiene masa $\alpha$. Sean $r_{1}$ la distancia entre las partículas de 
masa 1 y $r_{2}$ la distancia con signo entre el centro de masa de las partículas con masa 1 y $m_{2}$, véase la figura 1.4 (a).

Se trata de un problema con dos grados de libertad, cuya función potencial está dada por

$$
U\left(r_{1}, r_{2}\right)=\frac{1}{r_{1}}+\frac{4 \alpha}{\sqrt{r_{1}^{2}+4 r_{2}^{2}}},
$$

donde el primer término corresponde a la energía potencial de la interacción entre las partículas de masa 1, y el segundo término corresponde a la suma de las energías potenciales correspondiente a las interacciones de la masa $\alpha$, con cada una de las partículas de masa 1.

Luego el hamiltoniano del problema es

$$
H(\mathbf{r}, \mathbf{p})=\frac{1}{2} \mathbf{p}^{t} M^{-1} \mathbf{p}-\frac{1}{r_{1}}-\frac{4 \alpha}{\sqrt{r_{1}^{2}+4 r_{2}^{2}}},
$$

entonces la región de Hill está dada por el conjunto

$$
\left\{\mathbf{r} \in \mathbb{R}^{2} \mid h \geq-\frac{\sqrt{r_{1}^{2}+4 r_{2}^{2}}+4 \alpha r_{1}}{r_{1} \sqrt{r_{1}^{2}+4 r_{2}^{2}}}\right\} .
$$

Consideremos el caso $h<0$, entonces la región de Hill esta dada por la desigualdad

$$
-|h| \geq-\frac{1}{r_{1}}-\frac{4 \alpha}{\sqrt{r_{1}^{2}+4 r_{2}^{2}}},
$$

mediante el uso de técnicas elementales para desigualdades, no es difícil obtener que

$$
\frac{1}{|h|} \leq \mathbf{r}_{1} \leq \frac{4 \alpha+1}{|h|}
$$

lo cual nos dice que al tomar energías negativas, la distancia entre las partículas de masa 1 permanece acotada. Por otro lado, si ahora derivamos implícitamente la curva de velocidad cero $-U(\mathbf{r})=h$ con respecto a $\mathbf{r}_{2}$, obtenemos

$$
\mathbf{r}_{1}^{\prime}=\frac{d \mathbf{r}_{1}}{d \mathbf{r}_{2}}=-\frac{16 \mathbf{r}_{1}^{2}}{\left(\mathbf{r}_{1}^{2}+4 \mathbf{r}_{2}^{2}\right)^{3 / 2}+4 \alpha \mathbf{r}_{1}^{3}} \mathbf{r}_{2},
$$

lo cual quiere decir: cuando la distancia con signo $\mathbf{r}_{2}$ es positiva, entonces la derivada en cada punto de la curva de velocidad cero es negativa, y cuando la distancia con signo $\mathbf{r}_{2}$ es negativa, se tiene que la derivada en cada punto de la curva de velocidad cero, es positiva.

El análisis anterior nos da una idea de la región de Hill para el caso $h<0$, además utilizando el software Mathematica obtuvimos que los resultados encontrados anteriormente coinciden con la región dada por Mathematica, la cual se observa en la figura $1.4(b)$, esta región de Hill hace notar que cuando $h<0$, las partículas de masa 1 tiene movimiento acotado, mientras que la masa $\alpha$ puede escapar. Luego para $h \geq 0$ la región de Hill es el semiplano derecho de $\mathbb{R}^{2}$, esto es, las tres partículas tienen movimiento no acotado. Si el 


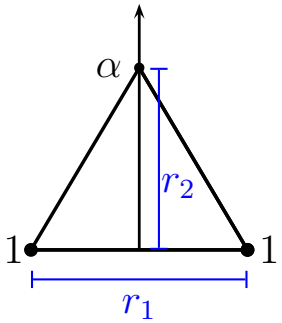

(a) Problema isósceles de los 3-cuerpos.

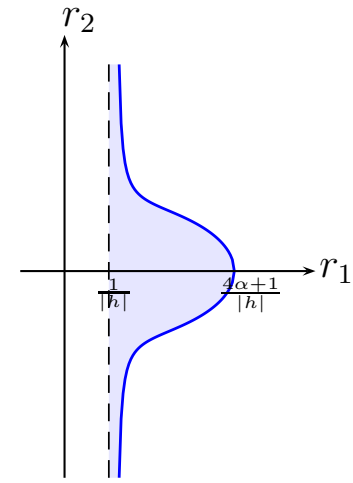

(b) $h<0$.

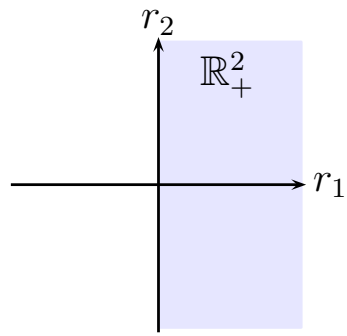

(c) Si $h \geq 0$ entonces la región de Hill es el semiplano derecho de $\mathbb{R}^{2}$.

Figura 1.4: Regiones de Hill en el problema isósceles de los tres cuerpos en el plano.

lector se encuentra interesado en conocer más acerca del problema isósceles de los 3-cuerpos vea $[16]$.

Observemos que las regiones de Hill depende únicamente de dos parámetros, el primero es el valor que se tome para la energía $h$ y el segundo la distancia entre las partículas, es por esto que las regiones de Hill son una herramienta muy útil para describir las regiones de movimiento del problema de los $N$-cuerpos, clasificando estos movimientos en acotados y no acotados.

Es interesante preguntarse si es posible introducir a las regiones de Hill un tercer parámetro, el cual este involucrado con la rotación de las partículas. Posteriormente en este trabajo surgirá de manera natural un concepto que denominamos regiones de Hill generalizadas; generalizadas en el sentido de que se introduce un tercer parámetro relacionado con los movimientos de rotación y que al igual que las regiones de Hill permitirá describir los movimientos en acotados y no acotados, aún cuando las partículas también experimenten movimientos de rotación.

\subsection{Cambio configuracional}

En este trabajo entendemos como cambio configuracional, a la transformación de la configuración inicial de las partículas para algún tiempo $t$, es decir, cuando las posiciones de las partículas sufren deformación; dicha deformación es diferente de tomar las posiciones y multiplicarlas por un mismo factor tal que éstas se contraigan o expandan permaneciendo sobre una línea recta que pase por el centro de masa, y además, diferente de rotar las partículas como cuerpos rígidos o una combinación de ambos movimientos, puesto que en ambos casos se sigue preservando la configuración. Entonces sucede que cuando nos referimos a cambio en la configuración, este será un cambio en la forma (deformación) que no contempla homotecias y rotaciones de ningún tipo. 


\subsubsection{Funciones homogéneas}

Las funciones homogéneas aparecen de manera natural y son una herramienta muy útil en el presente trabajo, a continuación las definimos brevemente y presentamos algunas propiedades y resultados que utilizamos en lo que sigue. A continuación se da la definición usual de una función homogénea.

Definición 1.4 Una función $f: \mathbb{R}^{N} \rightarrow \mathbb{R}$ y $f=f\left(x_{1}, \ldots, x_{N}\right)$, se dice homogénea de grado $\beta$ si

$$
f\left(t x_{1}, \ldots, t x_{N}\right)=t^{\beta} f\left(x_{1}, \ldots, x_{N}\right),
$$

para todo $t>0$.

Para las funciones homogéneas que admiten derivadas parciales continuas en su dominio se tiene un importante resultado conocido con el nombre de teorema de Euler.

Teorema 1.5 (Teorema de Euler) Sea $f: \mathbb{R}^{N} \rightarrow \mathbb{R}$ una función homogénea de grado $\beta$ y además $f \in C^{1}(\Omega)$. Entonces $f$ satisface

$$
\sum_{j=1}^{N} \frac{\partial f}{\partial x_{j}} \cdot x_{j}=\beta f .
$$

Demostración:

Escribiendo $\mathbf{x}=\left(x_{1}, \ldots, x_{N}\right)$ y diferenciando la ecuación

$$
f(t \mathbf{x})=t^{\beta} f(\mathbf{x})
$$

con respecto a $t$, encontramos aplicando la regla de la cadena que

$$
\frac{\partial f(t \mathbf{x})}{\partial t}=\frac{\partial}{\partial t}\left(t^{\beta} f(\mathbf{x})\right)
$$

entonces

$$
\frac{\partial}{\partial x_{1}} f(t \mathbf{x}) \frac{d}{d t}\left(t x_{1}\right)+\ldots+\frac{\partial}{\partial x_{N}} f(t \mathbf{x}) \frac{d}{d t}\left(t x_{N}\right)=\beta t^{\beta-1} f(\mathbf{x})
$$

Así que,

$$
x_{1} \frac{\partial}{\partial x_{1}} f(t \mathbf{x})+\ldots+x_{N} \frac{\partial}{\partial x_{N}} f(t \mathbf{x})=\beta t^{\beta-1} f(\mathbf{x}) .
$$

Como $f$ homogénea la expresión anterior se cumple para cualquier $t>0$, por lo que se seguirá cumpliendo para un valor fijo como $t=1$. Entonces sustituyendo $t=1$ se obtiene lo que se quiere demostrar, esto es,

$$
\beta f(\mathbf{x})=x_{1} \frac{\partial}{\partial x_{1}} f(\mathbf{x})+\ldots+x_{N} \frac{\partial}{\partial x_{N}} f(\mathbf{x})=\nabla f(\mathbf{x}) \cdot \mathbf{x}, \quad \nabla=\left(\frac{\partial}{\partial x_{1}}, \ldots, \frac{\partial}{\partial x_{N}}\right) .
$$

Otra propiedad interesante de las funciones homogéneas, se enuncia en el siguiente teorema. 
Teorema 1.6 Sean $f$ y $g$ dos funciones homogéneas de grado $\alpha$ y $\beta$, respectivamente. Entonces el producto de ambas es una función homogénea de grado $\alpha+\beta$.

Demostración:

$$
(f \cdot g)(t \mathbf{x})=f(t \mathbf{x}) \cdot g(t \mathbf{x})=t^{\alpha} f(\mathbf{x}) \cdot t^{\beta} g(\mathbf{x})=t^{\alpha} t^{\beta}[f(\mathbf{x}) \cdot g(\mathbf{x})]=t^{\alpha+\beta}(f \cdot g)(x) .
$$

Como hemos visto es fácil saber si una función es homogénea de grado $\beta$, por ejemplo, consideremos la energía potencial al cuadrado, $U^{2}$, se verifica fácilmente que es una función homogénea de grado -2 , es decir, satisface la condición

$$
U^{2}\left(t \mathbf{r}_{1}, \ldots, t \mathbf{r}_{N}\right)=t^{-2} U^{2}\left(\mathbf{r}_{1}, \ldots, \mathbf{r}_{N}\right),
$$

esto nos dice que la función potencial es sensible a cambios en la escala de la configuración de las partículas.

Otro ejemplo es la función momento de inercia, $I$, definido por la ecuación (1.7), la cual es una función homogénea de grado 2, es decir, se cumple

$$
I\left(t \mathbf{r}_{1}, \ldots, t \mathbf{r}_{N}\right)=t^{2} I\left(\mathbf{r}_{1}, \ldots, \mathbf{r}_{N}\right) .
$$

Por lo tanto, por el teorema 1.6 al multiplicar ambas funciones homogéneas, $I U^{2}$, obtenemos una función homogénea de grado cero, esto es,

$$
I U^{2}\left(t \mathbf{r}_{1}, \ldots, t \mathbf{r}_{N}\right)=I U^{2}\left(\mathbf{r}_{1}, \ldots, \mathbf{r}_{N}\right),
$$

lo cual significa que el valor de la función homogénea no depende del tamaño de la configuración definida por las partículas, es decir, la función $I U^{2}$ es invariante ante homotecias. Más aún esta función homogénea de grado cero también es invariante ante rotaciones, puesto que $I U^{2}$ se define estrictamente en términos de las distancias mutuas entre las partículas, ya que tanto $I(\mathbf{r})$ como $U(\mathbf{r})$ están expresadas en términos de las distancias mutuas entre las partículas.

Entonces como la función $I U^{2}$ es invariante ante contracciones, expansiones y rotaciones, ésta es vista como una forma de medir el cambio configuracional, es decir, $I U^{2}$ mide cómo la forma de la configuración está cambiando y la llamamos, la medida configuracional. Lo anterior nos permite dar la siguiente definición.

Definición 1.7 La medida configuracional del sistema

$$
m_{j} \ddot{\mathbf{r}}_{j}=\sum_{i \neq j, i=1}^{N} \frac{m_{i} m_{j}}{\left|\mathbf{r}_{i}-\mathbf{r}_{j}\right|^{3}}\left(\mathbf{r}_{i}-\mathbf{r}_{j}\right), \quad j=1, \ldots, N,
$$

está dada por IU².

Más adelante en este trabajo, veremos que la función $I U^{2}$ está relacionada con una de las tres componentes de la descomposición de la velocidad de Saari. En el siguiente apartado estudiaremos la relación que existe entre esta medida configuracional $I U^{2}$, y una importante desigualdad de mecánica celeste. 


\subsubsection{Desigualdad de Sundman y restricciones del movimiento}

Karl F. Sundman fue un matemático finlandés que contribuyo con grandes resultados al estudio del problema de los $N$-cuerpos, en esta sección introducimos una desigualdad la cual lleva su nombre, dicha desigualdad es fundamental pues conecta los conceptos energía cinética, momento de inercia y momento angular.

Tomando el módulo del momento angular

$$
|\mathbf{c}|=\left|\sum_{j=1}^{N} m_{j} \mathbf{r}_{j} \times \mathbf{v}_{j}\right| \leq \sum_{j=1}^{N} m_{j}\left|\mathbf{r}_{j} \times \mathbf{v}_{j}\right|=\sum_{j=1}^{N} m_{j} r_{j} v_{j}\left|\operatorname{sen} \theta_{j}\right|
$$

donde $r_{j}=\left|\mathbf{r}_{j}\right|, v_{j}=\left|\mathbf{v}_{j}\right|$ y $\theta_{j}$ es el ángulo entre los vectores $\mathbf{r}_{j} \mathrm{y} \mathbf{v}_{j}$.

Haciendo $c=|\mathbf{c}|$ hemos obtenido,

$$
c \leq \sum_{j=1}^{N} m_{j} r_{j} v_{j}\left|\operatorname{sen} \theta_{j}\right|
$$

Tomando el módulo y elevando al cuadrado la desigualdad anterior

$$
|c|^{2} \leq\left|\sum_{j=1}^{N} m_{j} r_{j} v_{j}\right| \operatorname{sen} \theta_{j}||^{2}=\left|\sum_{j=1}^{N}\left(\sqrt{m_{j}} r_{j}\right)\left(\sqrt{m_{j}} v_{j}\left|\operatorname{sen} \theta_{j}\right|\right)\right|^{2}
$$

aplicando la desigualdad de Schwarz

$$
c^{2} \leq \sum_{j=1}^{N} m_{j} r_{j}^{2} \sum_{j=1}^{N} m_{j} v_{j}^{2} \operatorname{sen}^{2} \theta_{j}
$$

Por otro lado, derivando el momento de inercia del sistema, $I=\sum_{j=1}^{N} m_{j}\left(\mathbf{r}_{j} \cdot \mathbf{r}_{j}\right)$, con respecto al tiempo

$$
\dot{I}=2 \sum_{j=1}^{N} m_{j}\left(\mathbf{r}_{j} \cdot \mathbf{v}_{j}\right)=2 \sum_{j=1}^{N} m_{j} r_{j} v_{j} \cos \theta_{j},
$$

tomando el módulo y elevando al cuadrado

$$
|\dot{I}|^{2}=\left|\sum_{j=1}^{N}\left(2 \sqrt{m_{j}} r_{j}\right)\left(\sqrt{m_{j}} v_{j} \cos \theta_{j}\right)\right|^{2},
$$

aplicando nuevamente la desigualdad de Schwarz a la ecuación anterior

$$
\dot{I}^{2} \leq 4 \sum_{j=1}^{N} m_{j} r_{j}^{2} \sum_{j=1}^{N} m_{j} v_{j}^{2} \cos ^{2} \theta_{j}
$$


Sumando las ecuaciones (1.15) y (1.16) tenemos

$$
\frac{\dot{I}^{2}}{4}+c^{2} \leq \sum_{j=1}^{N} m_{j} r_{j}^{2}\left(\sum_{j=1}^{N} m_{j} v_{j}^{2} \operatorname{sen}^{2} \theta_{j}+\sum_{j=1}^{N} m_{j} v_{j}^{2} \cos ^{2} \theta_{j}\right)=\sum_{j=1}^{N} m_{j} r_{j}^{2} \sum_{j=1}^{N} m_{j} v_{j}^{2} .
$$

Por lo tanto, obtenemos la desigualdad de Sundman

$$
\frac{\dot{I}^{2}}{4}+c^{2} \leq I T
$$

Sabemos que existen herramientas muy útiles para describir las regiones de movimiento del problema de los $N$-cuerpos, tales como las curvas de velocidad cero y las regiones de Hill. Lo que haremos a continuación es describir restricciones similares en el movimiento posible de las partículas, las cuales son válidas para el problema de los $N$-cuerpos, [10].

Como $I U^{2}$ mide el cambio en la forma de la configuración, sí podemos encontrar una restricción de los valores posibles que puede tomar $I U^{2}$, esto se traduciría como una restricción para las configuraciones posibles que las $N$-partículas podrían formar.

Entonces consideremos la desigualdad de Sundman (1.17) y la integral de energía total del sistema $T=U+h$, de aquí podemos escribir

$$
c^{2} \leq c^{2}+\dot{I}^{2} \leq 4 I(U+h)
$$

Dividiendo ambos lados de la desigualdad anterior por $4 I^{1 / 2}$

$$
\frac{c^{2}}{4 I^{1 / 2}} \leq \frac{c^{2}+\dot{I}^{2}}{4 I^{1 / 2}} \leq \frac{4 I}{4 I^{1 / 2}}(U+h),
$$

y restando $h I^{1 / 2}$ del resultado obtenido, la desigualdad de Sundman toma la forma

$$
\frac{c^{2}}{4 I^{1 / 2}}-h I^{1 / 2} \leq \frac{c^{2}+\dot{I}^{2}}{4 I^{1 / 2}}-h I^{1 / 2} \leq I^{1 / 2} U
$$

Esto significa que las constantes del sistema $c, h$ y $I^{1 / 2}$ imponen un limite inferior a $I^{1 / 2} U$. Para analizar esto restringiremos nuestra atención al caso más interesante $h<0$. Pues si $h \geq 0$ no hay limite inferior efectivo para $I^{1 / 2} U$. Sin embargo, incluso si $h \geq 0$, las restricciones sobre $I^{1 / 2} U$ ocurren si $I^{1 / 2}$ tiene un valor suficientemente pequeño.

Así analizamos las posibles restricciones para los valores de $I^{1 / 2} U$ cuando $h<0$, aquí esas restricciones son independiente del valor de $I^{1 / 2}$, haciendo que éstas dependan solamente del valor de $c^{2}|h|$. Mediante el uso de técnicas de cálculo elemental veamos que esto es cierto. Sea

$$
f\left(I^{1 / 2}\right)=\frac{c^{2}+\dot{I}^{2}}{4 I^{1 / 2}}+|h| I^{1 / 2}, \quad \text { con } \quad c \neq 0 \quad \text { y } \quad h<0 .
$$

Veamos para qué valores de $I^{1 / 2}$ la función $f\left(I^{1 / 2}\right)$ alcanza un máximo o mínimo. Tomemos

$$
f^{\prime}\left(I^{1 / 2}\right)=-\frac{c^{2}}{4\left(I^{1 / 2}\right)^{2}}+|h|
$$


igualando a cero y resolviendo la ecuación anterior se tiene

$$
I^{1 / 2}=\frac{c}{2|h|^{1 / 2}}
$$

Ahora, puesto que

$$
f^{\prime \prime}\left(I^{1 / 2}\right)=\frac{c^{2}}{2\left(I^{1 / 2}\right)^{3}} \quad \text { y } \quad f^{\prime \prime}\left(\frac{c}{2|h|^{1 / 2}}\right)=\frac{4|h|^{3 / 2}}{c}>0,
$$

entonces la gráfica de $f\left(I^{1 / 2}\right)$ alcanza un valor mínimo en $I^{1 / 2}=\frac{c}{2|h|^{1 / 2}}$, el cual es

$$
f\left(\frac{c}{2|h|^{1 / 2}}\right)=c|h|^{1 / 2}
$$

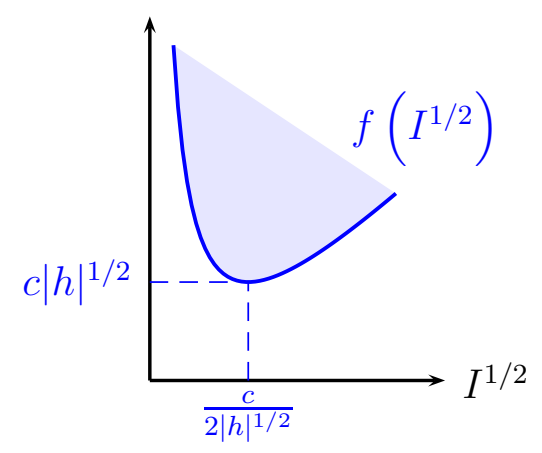

Figura 1.5: Posibles valores de $I^{1 / 2} U$.

Por lo tanto, los valores posibles de $I^{1 / 2} U$ están restringidos a encontrarse en la región por encima o sobre la gráfica de $f\left(I^{1 / 2}\right)$ como se muestra en la figura 1.5. En particular el valor de $I^{1 / 2} U$ está acotado inferiormente por el mínimo valor de la curva. Entonces para valores de la energía $h<0$ siempre es cierto que

$$
c|h|^{1 / 2} \leq I^{1 / 2} U,
$$

donde este límite inferior ocurre si y sólo si $I^{1 / 2}=\frac{c}{2|h|^{1 / 2}}$.

Luego elevando al cuadrado la desigualdad anterior también podemos escribir

$$
c^{2}|h| \leq I U^{2} .
$$

Por lo tanto, la desigualdad de la ecuación (1.19) es una restricción de las posibles configuraciones del problema de los $N$-cuerpos, la cual es independiente del valor de $I$, es decir, para valores de la energía $h<0$ la función que mide cómo cambia la configuración de las partículas, $I U^{2}$, está acotada inferiormente por el mínimo $c^{2}|h|$. 


\subsection{Configuraciones centrales y soluciones homográficas}

El propósito de esta sección es estudiar la existencia de algunas soluciones particulares, es sabido que para un problema newtoniano, se conocen soluciones especiales: las generadas por configuraciones centrales, las cuales definiremos más adelante. El estudio de las configuraciones centrales inicia en el año de 1767 cuando el matemático y físico suizo Leonhard Euler publica un interesante trabajo sobre el problema de los 3-cuerpos, en aquella época los investigadores estaban bastante interesados en el estudió de órbitas periódicas. En su trabajo, Euler encuentra explícitamente una familia completa de ellas, concretamente demuestra que si tres partículas con masas arbitrarias son colocadas inicialmente en línea recta, de tal forma que la razón de sus distancias satisfaga una fórmula que sólo depende del valor de las masas, y si además, las velocidades iniciales son escogidas adecuadamente, entonces cada partícula se moverá periódicamente sobre una elipse, pero en todo momento las tres partículas se mantendrán sobre una línea recta, conservando siempre la misma razón entre sus distancias.

Unos años después, en 1772, Joseph L. Lagrange encuentra una nueva familia de órbitas periódicas, obtenidas al colocar tres masas arbitrarias sobre los vértices de un triángulo equilátero, donde hay que escoger adecuadamente las velocidades iniciales. Aquí nuevamente cada partícula se moverá sobre una órbita elíptica; en todo momento la configuración será de triángulo equilátero, el cual podrá variar de tamaño pero nunca de forma. La configuración inicial de la cual parten tanto Euler como Lagrange es una configuración central. El estudio de las configuraciones centrales es de primordial importancia en la mecánica celeste, en 1912 Sundman demostró que en el problema de los 3-cuerpos, cuando las partículas chocan simultáneamente (colisión triple) lo hacen acercándose a una configuración central; este resultado ha sido generalizado y hoy en día se ha demostrado que en el problema de los $N$-cuerpos, cualquier colisión simultanea de $k$-partículas con $3 \leq k \leq N$, se acerca de forma asintótica a una configuración central formada por esas $k$-partículas. También se sabe que en cualquier problema de los $N$-cuerpos $(N \geq 3)$ existe al menos una configuración central.

Pero sin duda, la principal aportación de las configuraciones centrales, es que ellas generan las únicas soluciones explícitas del problema de los $N$-cuerpos, llamadas soluciones homográficas, más adelante en esta sección retomaremos el estudio de éstas. Por este solo hecho, el estudio de las configuraciones centrales es de primordial importancia en la mecánica celeste.

A continuación se da la definición usual de configuración central en un sistema de $\mathrm{N}$ partículas.

Definición 1.8 Sea $\mathbf{r}=\left(\mathbf{r}_{1}, \mathbf{r}_{2}, \ldots, \mathbf{r}_{N}\right)$ la configuración del sistema

$$
m_{j} \ddot{\mathbf{r}}_{j}=\frac{\partial U}{\partial \mathbf{r}_{j}}, \quad j=1, \ldots, N .
$$

Decimos que $\mathbf{r}$ es una configuración central si

$$
\ddot{\mathbf{r}}_{j}=\lambda(t) \mathbf{r}_{j}, \quad \text { para } \quad j=1, \ldots, N,
$$

donde $\lambda(t)$ es una función escalar, la cual es la misma para todas las partículas. 
En otras palabras, una configuración central son posiciones particulares de las $N$-partículas donde los vectores de posición y aceleración de cada partícula son proporcionales, con la misma constante de proporcionalidad.

Utilizando la ecuación de movimiento (1.20), tenemos que la configuración central satisface

$$
\lambda m_{j} \mathbf{r}_{j}=\frac{\partial U}{\partial \mathbf{r}_{j}}, \quad \text { para } \quad j=1, \ldots, N
$$

Por otro lado, tenemos

$$
\nabla I=\frac{\partial I}{\partial \mathbf{r}_{j}}=m_{j} \mathbf{r}_{j}
$$

lo cual indica que una configuración central se expresa en términos de los multiplicadores de Lagrange (siempre y cuando se fije el momento de inercia), es decir, encontrar $\lambda$ tal que

$$
\lambda \nabla I=\nabla U
$$

Al tomar el producto punto de ambos lados de la ecuación (1.21) con el vector $\mathbf{r}_{j}$ se obtiene

$$
\lambda m_{j} \mathbf{r}_{j} \cdot \mathbf{r}_{j}=\frac{\partial U}{\partial \mathbf{r}_{j}} \cdot \mathbf{r}_{j}
$$

entonces sumando sobre todo $j$

$$
\lambda \sum_{j=1}^{N} m_{j}\left|\mathbf{r}_{j}\right|^{2}=2 \lambda I=\sum_{j=1}^{N} \frac{\partial U}{\partial \mathbf{r}_{j}} \cdot \mathbf{r}_{j}
$$

y al ser $U$ homogénea de grado -1 entonces por el teorema 1.5 (teorema de Euler)

$$
2 \lambda I=-U
$$

Sustituyendo el valor establecido para $\lambda$ en la ecuación (1.22), dicha expresión se cumple si y sólo si

$$
\begin{gathered}
-\frac{U}{2 I} \nabla I=\nabla U \\
\Leftrightarrow \quad 2 I \nabla U+U \nabla I=0, \\
\Leftrightarrow \quad \nabla(I U)+I \nabla U=0 .
\end{gathered}
$$

Multiplicando la igualdad anterior por $U$ y aplicando la fórmula de la derivada de un producto se obtiene

$$
\nabla\left(I U^{2}\right)=0
$$

A continuación enunciamos los dos anteriores resultados en el siguiente teorema. 
Teorema 1.9 Para una configuración central,

$$
\lambda=-\frac{U}{2 I} .
$$

Una configuración $\mathbf{r}=\left(\mathbf{r}_{1}, \ldots \mathbf{r}_{N}\right)$ es central si y sólo si

$$
\nabla I U^{2}(\mathbf{r})=0 .
$$

Este teorema nos afirma en primer lugar que la función escalar $\lambda(t)$ siempre es negativa, es decir, $\lambda(t)<0$ y que una configuración central es un punto crítico de la función que mide cómo cambia la forma de la configuración de las partículas, $I U^{2}$.

Una clase especial de soluciones que nos gustaría encontrar en nuestro problema son las soluciones homográficas.

Definición 1.10 Una solución $\mathbf{r}(t)$ del problema de los $N$-cuerpos se llama homográfica si la configuración $\left(\mathbf{r}_{1}(t), \ldots, \mathbf{r}_{N}(t)\right) \in\left(\mathbb{R}^{3}\right)^{N}$ sigue siendo la misma para todo tiempo $t$. Es decir, una solución homográfica está caracterizada por la existencia de una función escalar $R(t)>0$ y una matriz de rotación $\Omega(t) \in S O(3)$ tal que para cada $j$ y para todo tiempo $t$, se tiene

$$
\mathbf{r}_{j}(t)=R(t) \Omega(t) \mathbf{r}_{j}\left(t_{0}\right),
$$

donde $t_{0}$ es un momento fijo en el tiempo y $\mathbf{r}_{j}\left(t_{0}\right)$ es una configuración central.

Existen dos casos límite de las soluciones homográficas, los cuales se analizan enseguida:

- El primero cuando $\Omega(t)$ es la matriz identidad, es decir,

$$
\mathbf{r}_{j}(t)=R(t) \mathbf{r}_{j}\left(t_{0}\right), \quad j=1, \ldots, N,
$$

entonces las soluciones se llaman homotéticas. Estas soluciones permiten que las partículas cambien el tamaño en la escala preservando al mismo tiempo la configuración, pero sin rotación de ningún tipo. Esto significa que cada partícula está restringida a permanecer sobre una línea recta que pasa por centro de masa del sistema. De acuerdo a la definición de momento angular de un sistema de partículas, el comportamiento homotético puede ocurrir solamente si $\mathbf{c}=0$.

- El segundo caso se tiene cuando $R(t)=1$, esto es,

$$
\mathbf{r}_{j}(t)=\Omega(t) \mathbf{r}_{j}\left(t_{0}\right), \quad j=1, \ldots, N,
$$

entonces las soluciones se llaman equilibrios relativos.

Las segundas se comportan como un cuerpo rígido en rotación, es decir, las distancias entre todos los cuerpos permanecen fijas por lo que las partículas giran sin que haya cambio en la escala. Esto es, el sistema rota al rededor del centro de masa como si se tratara de un cuerpo rígido, las distancias mutuas no cambian cuando el tiempo $t$ varía, cada partícula se mueve exactamente en una órbita idéntica. El nombre de equilibrio 
relativo viene del hecho de que si a nuestro sistema de referencia lo hacemos rotar alrededor del centro de masa, entonces en el nuevo sistema, las partículas permanecen en equilibrio o reposo. Las soluciones de equilibrio relativo son órbitas periódicas del sistema (1.3), puesto que al girar las partículas como un cuerpo rígido este equilibrio relativo es un punto fijo y podemos estudiar su estabilidad.

Finalmente, en este primer capítulo hemos estudiado algunos conceptos y resultados conocidos del problema de los $N$-cuerpos, los cuales vincularemos más adelante con aspectos relacionados a la descomposición de la velocidad de Saari. 
CAPÍTULO 2

\section{Descomposición de la velocidad de Saari}

\subsection{Antecedentes}

Sabemos que en la actualidad el problema de los $N$-cuerpos con $N \geq 3$, tiene aún muchas preguntas por contestar y que este problema continua siendo un misterio apasionante y complejo. Por la importancia e interés que existen en comprender la dinámica tan complicada del problema de los $N$-cuerpos, el objetivo de este segundo capítulo es el estudio de la descomposición de la velocidad del problema de los $N$-cuerpos, la cual fue introducida por el matemático Donald G. Saari en los años setenta, [11], [12], [13] y [14]. La idea de Saari de descomponer la velocidad total de un sistema de $N$-partículas moviéndose bajo sus atracciones gravitacionales, fue motivada por la necesidad de encontrar nuevas herramientas para entender mejor la dinámica tan complicada del problema de los $N$-cuerpos en general, y la de los tres cuerpos en particular, en especial en el estudio de los movimientos acotados. Para dar inicio al estudio de la descomposición de la velocidad de Saari, primero damos a conocer la idea geométrica de la cual partió su análisis.

Sean $\mathbf{r}_{j}, \mathbf{v}_{j}=\dot{\mathbf{r}}_{j}$ y $m_{j}$ la posición, la velocidad y la masa de la $j$-ésima partícula, respectivamente, entonces las ecuaciones de movimiento son

$$
m_{j} \ddot{\mathbf{r}}_{j}=\sum_{i \neq j, i=1}^{N} \frac{m_{i} m_{j}}{\left|\mathbf{r}_{i}-\mathbf{r}_{j}\right|^{3}}\left(\mathbf{r}_{i}-\mathbf{r}_{j}\right)=\frac{\partial U}{\partial \mathbf{r}_{j}}
$$

donde el potencial del sistema está definido como

$$
U=\sum_{i<j} \frac{m_{j} m_{i}}{\left|\mathbf{r}_{i}-\mathbf{r}_{j}\right|}
$$


Consideremos los vectores de posición y velocidad del sistema, los cuales definimos de la siguiente manera, $\mathbf{r}=\left(\mathbf{r}_{1}, \mathbf{r}_{2}, \ldots, \mathbf{r}_{N}\right)$ y $\mathbf{v}=\left(\mathbf{v}_{1}, \mathbf{v}_{2}, \ldots, \mathbf{v}_{N}\right)$, respectivamente. Pensemos en una solución al sistema anterior y tomemos el vector velocidad $\mathbf{v}$, Saari tuvo la idea de descomponer el vector de velocidad de tal forma que esta descomposición permitiera conocer información sobre cómo la velocidad influye (afecta) en las rotaciones del sistema, en el cambio del tamaño del sistema y en cómo cambia la forma del sistema, es decir, obtener las componentes de la velocidad que cambia la orientación de las partículas vistas como un cuerpo rígido, las componentes de la velocidad que cambia la escala de la configuración y las componentes de la velocidad que deforma la configuración, respectivamente.

Para obtener geométricamente la primer componente de la velocidad, encargada de cambiar la orientación de las partículas vistas como un cuerpo rígido, Saari realizó proyecciones del vector de velocidad del sistema $\mathbf{v}$, a cada uno de los tres ejes independientes de rotación para la configuración. Luego, para obtener la segunda componente realizó nuevamente proyecciones de $\mathbf{v}$ a lo largo de un vector unitario, el cual representa el tamaño del sistema (véase la figura 2.1). La tercer componente sin embargo resulta ser la más complicada de obtener, pero una vez encontradas las dos primeras, es posible definir una expresión para la tercer componente en términos de las dos primeras. Ahora, Saari se percato que lo que había realizado geométricamente, lo podia obtener definiendo un producto escalar (producto interno), es decir, Saari definió un producto escalar para el sistema de los $N$-cuerpos, y con respecto a éste descompuso ortogonalmente el vector de velocidad del sistema.

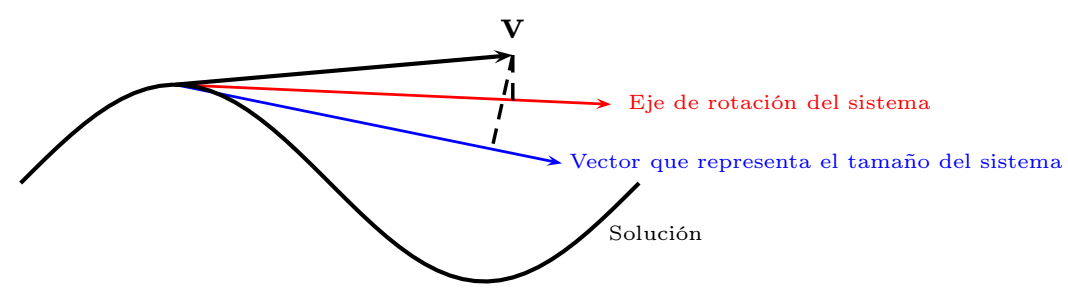

Figura 2.1: Interpretación geométrica de la descomposición de la velocidad de Saari.

Es importante mencionar (recordar) que en un espacio vectorial con producto interno, dados cuales quiera dos vectores distintos de cero, es decir, $\mathbf{a}, \mathbf{b} \in V$, entonces la proyección de $\mathbf{a}$ sobre $\mathbf{b}$ es un vector denotado por $\operatorname{proy}_{\mathbf{b}} \mathbf{a}$ (ver figura 2.2), que se define por

$$
\operatorname{proy}_{\mathbf{b}} \mathbf{a}=\frac{\mathbf{a} \cdot \mathbf{b}}{|\mathbf{b}|} \frac{\mathbf{b}}{|\mathbf{b}|},
$$

donde la componente de $\mathbf{a}$ en dirección de $\mathbf{b}$ es $\frac{\mathbf{a} \cdot \mathbf{b}}{|\mathbf{b}|}$, y es un escalar.

A continuación definimos un producto escalar, y además un producto vectorial para el sistema de los $N$-cuerpos.

Dados $\mathbf{A}, \mathbf{B} \in\left(\mathbb{R}^{3}\right)^{N}$, el producto escalar y producto vectorial para el sistema de los $N$-cuerpos son

$$
\langle\mathbf{A}, \mathbf{B}\rangle=\sum_{j=1}^{N} m_{j}\left(\mathbf{a}_{j}, \mathbf{b}_{j}\right)
$$




$$
\mathbf{A} \times \mathbf{B}=\left(\mathbf{a}_{1} \times \mathbf{b}_{1}, \ldots, \mathbf{a}_{N} \times \mathbf{b}_{N}\right),
$$

respectivamente, donde $\left(\mathbf{a}_{j}, \mathbf{b}_{j}\right)$ y $\mathbf{a}_{j} \times \mathbf{b}_{j}$ son el producto escalar y el producto vectorial usual en $\mathbb{R}^{3}$, respectivamente. Sea $\mathbf{E}_{i}=\left(\mathbf{e}_{i}, \ldots, \mathbf{e}_{i}\right) \in\left(\mathbb{R}^{3}\right)^{N}$, donde $\mathbf{e}_{i}$ es el clásico vector unitario en $\mathbb{R}^{3}$ con valor unitario en la $i$-ésima componente.

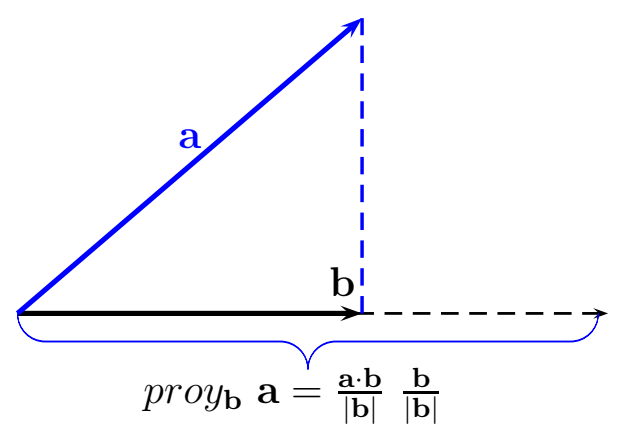

Figura 2.2: La proyección de $\mathbf{a}$ sobre $\mathbf{b}$ es el vector $\operatorname{proy}_{b} a$.

El producto escalar y vectorial del sistema permiten escribir en forma compacta el momento de inercia y las integrales de movimiento, es decir, éstos simplifican la notación; por ejemplo para el momento de inercia tenemos:

Denotando al $j$-ésimo vector de posición por $\mathbf{r}_{j}=\left(x_{j}, y_{j}, z_{j}\right) \in \mathbb{R}^{3}$, entonces el momento de inercia bajo el producto escalar del sistema se escribe como

$$
I=\sum_{j=1}^{N} m_{j}\left|\mathbf{r}_{j}\right|^{2}=\sum_{j=1}^{N} m_{j}\left(\mathbf{r}_{j}, \mathbf{r}_{j}\right)=\langle\mathbf{r}, \mathbf{r}\rangle .
$$

Luego, desarrollando la forma acostumbrada del centro de masa

$$
\begin{aligned}
\overline{0} & =\sum_{j=1}^{N} m_{j} \mathbf{r}_{j} \\
& =\left(m_{1} x_{1}+\ldots+m_{N} x_{N}, m_{1} y_{1}+\ldots+m_{N} y_{N}, m_{1} z_{1}+\ldots+m_{N} z_{N}\right) \\
& =\left(m_{1}\left(\mathbf{r}_{1}, \mathbf{e}_{1}\right)+\ldots+m_{N}\left(\mathbf{r}_{N}, \mathbf{e}_{1}\right), m_{1}\left(\mathbf{r}_{1}, \mathbf{e}_{2}\right)+\ldots+m_{N}\left(\mathbf{r}_{N}, \mathbf{e}_{2}\right),\right. \\
& =\left(\sum_{j=1}^{N} m_{j}\left(\mathbf{r}_{j}, \mathbf{e}_{1}\right), \sum_{j=1}^{N} m_{j}\left(\mathbf{r}_{j}, \mathbf{e}_{2}\right), \sum_{j=1}^{N} m_{j}\left(\mathbf{r}_{j}, \mathbf{e}_{3}\right)\right)=\left(\left\langle\mathbf{r}, \mathbf{E}_{1}\right\rangle,\left\langle\mathbf{r}, \mathbf{E}_{2}\right\rangle,\left\langle\mathbf{r}, \mathbf{E}_{3}\right\rangle\right) .
\end{aligned}
$$

Por lo tanto, la $i$-ésima componente del centro de masa restringido al producto escalar del sistema es

$$
\left\langle\mathbf{r}, \mathbf{E}_{i}\right\rangle=0, \quad i=1,2,3
$$

Estamos describiendo el sistema de los $N$ cuerpos en el espacio de configuración $\left(\mathbb{R}^{3}\right)^{N}$, sabemos que al restringir el centro de masa al origen (ecuación (2.2)) se reduce la dimensión 
del espacio de configuración, denotemos entonces al subespacio lineal definido por la ecuación (2.2) por $\left(\mathbb{R}^{3}\right)^{N-1}$, y su espacio tangente por $T_{\mathbf{r}}\left(\mathbb{R}^{3}\right)^{N-1} \equiv\left(\mathbb{R}^{3}\right)^{N-1}$. La unión de todos los espacios tangentes $T_{\mathbf{r}}\left(\mathbb{R}^{3}\right)^{N-1}$, es el haz tangente de $\left(\mathbb{R}^{3}\right)^{N-1}$, en el cual vive el vector de velocidad del sistema.

Con respecto al momento angular cuya expresión está dada por la ecuación (1.12), utilizando propiedades usuales del producto escalar y vectorial en $\mathbb{R}^{3}$, la $i$-ésima componente del momento angular cumple

$$
c_{i}=\left(\mathbf{c}, \mathbf{e}_{i}\right)=\left(\sum_{j=1}^{N} m_{j} \mathbf{r}_{j} \times \mathbf{v}_{j}, \mathbf{e}_{i}\right)=\sum_{j=1}^{N} m_{j}\left(\mathbf{e}_{i}, \mathbf{r}_{j} \times \mathbf{v}_{j}\right)=\left\langle\mathbf{E}_{i} \times \mathbf{r}, \mathbf{v}\right\rangle,
$$

de aquí, el vector momento angular toma la forma

$$
\mathbf{c}=\left(c_{1}, c_{2}, c_{3}\right)=\left(\left\langle\mathbf{E}_{1} \times \mathbf{r}, \mathbf{v}\right\rangle,\left\langle\mathbf{E}_{2} \times \mathbf{r}, \mathbf{v}\right\rangle,\left\langle\mathbf{E}_{3} \times \mathbf{r}, \mathbf{v}\right\rangle\right)
$$

La ecuación (2.3) hace ver que la componente (de la proyección) de $\mathbf{v}$ a lo largo de la linea que contiene al vector $\mathbf{E}_{i} \times \mathbf{r}$ es una constante. La figura 2.3 muestra la situación. Esta primera proyección del vector de velocidad es el inicio de la estrategia que sugirió Saari para descomponer ortogonalmente el vector de velocidad del sistema.

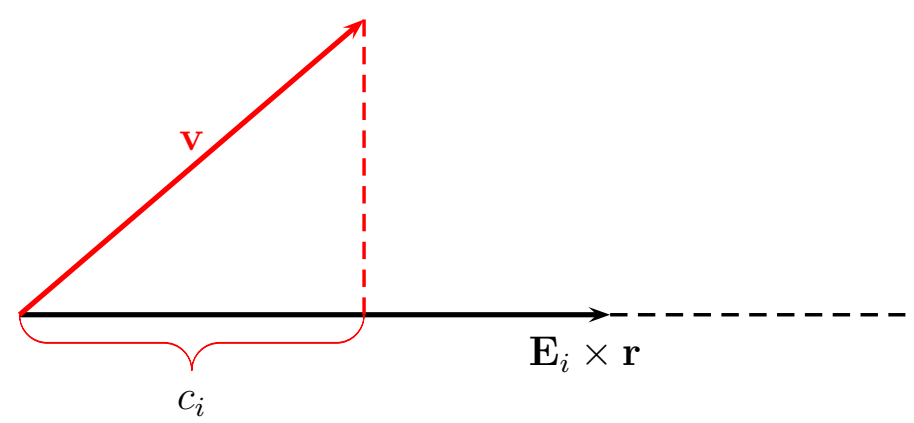

Figura 2.3: La componente de $\mathbf{v}$ a lo largo del vector $\mathbf{E}_{i} \times \mathbf{r}$ es $c_{i}$, y es un escalar.

La energía total del sistema en su forma tradicional se escribe como

$$
T-U=h
$$

donde $T$ es la energía cinética dada por

$$
T=\frac{1}{2} \sum_{j=1}^{N} m_{j}\left|\mathbf{v}_{j}\right|^{2}=\frac{1}{2} \sum_{j=1}^{N} m_{j}\left(\mathbf{v}_{j}, \mathbf{v}_{j}\right)=U+h,
$$

por tanto, la integral de energía total restringida al producto escalar del sistema es

$$
\langle\mathbf{v}, \mathbf{v}\rangle=2(U+h)
$$


Una descomposición ortogonal natural del vector velocidad del sistema es,

$$
\mathbf{v}=W_{1}+W_{2}+W_{3}
$$

donde la velocidad rotacional $W_{1}$, está relacionada con las rotaciones del sistema visto como un cuerpo rígido, la velocidad escalar $W_{2}$ representa el cambio en el tamaño del sistema, y la velocidad configuracional $W_{3}$ es la porción de $\mathbf{v}$ dedicada al cambio en la configuración definida por las partículas.

La descomposición ortogonal del vector de velocidad del sistema, se refiere a lo siguiente: dos subespacios vectoriales $V$ y $W$ de un espacio vectorial se dicen ortogonales si $\langle\mathbf{v}, \mathbf{w}\rangle=0$ para todo vector $\mathbf{v} \in V$ y $\mathbf{w} \in W$. En este caso, decimos que $V$ es el complemento ortogonal de $W$ y lo denotamos por $V=W^{\perp}$ (y $W$ es también el complemento ortogonal de $V$ ). Dicho de otra forma: el complemento ortogonal de $W$ es el conjunto de todos los vectores $\mathbf{v}$ que son perpendiculares a $W$,

$$
W^{\perp}=\left\{\mathbf{v} \in \mathbb{R}^{n} \mid\langle\mathbf{v}, \mathbf{w}\rangle=0, \quad \text { para todo } \quad \mathbf{w} \in W\right\} .
$$

Para comprobar si un vector $\mathbf{v}$ pertenece al complemento ortogonal de un espacio dado $W$, basta con comprobar si $\mathbf{v}$ es ortogonal a un conjunto que genere a $W$.

Luego, si $W$ es un subespacio de dimensión finita del espacio vectorial $V$, con producto interno y supongamos que $\mathbf{v} \in V$. Entonces, existen vectores únicos $\mathbf{w} \in W$ y $\mathbf{w}^{\perp} \in W^{\perp}$ tales que $v$ se descompone ortogonalmente como $\mathbf{v}=\mathbf{w}+\mathbf{w}^{\perp}$, donde $\mathbf{w}=$ proy $_{W} \mathbf{v}$ y si $V$ tiene dimensión finita, entonces $\mathbf{w}^{\perp}=\operatorname{proy}_{W} \perp \mathbf{v}$.

Ahora estamos listos para iniciar el estudio de la descomposición de la velocidad de un sistema de $N$ partículas, la cual divide el espacio tangente $T_{\mathbf{r}}\left(\mathbb{R}^{3}\right)^{N-1}$ en tres subespacios ortogonales naturales, donde las dos primeras componentes $W_{1}$ y $W_{2}$ determinan cambios en la orientación y tamaño escalar de una configuración, respectivamente, mientras que el vector final $W_{3}$, es la componente de la velocidad dedicada al cambio en la forma de la configuración, la cual resulta ser la más complicada.

En el siguiente apartado estudiaremos con detalle cada una de las tres componentes de la velocidad.

\subsection{Componentes de la velocidad, $W_{1}, W_{2} \mathbf{y} W_{3}$}

\section{Velocidad rotacional}

La componente de la velocidad dedicada a las rotaciones del sistema, $W_{1}$, es la proyección de $\mathbf{v}$ al subespacio generado por la base conformada específicamente por tres ejes independientes de rotación para la configuración,

$$
\left\{\mathbf{E}_{i} \times \mathbf{r}:=\left(\mathbf{e}_{i} \times \mathbf{r}_{1}, \ldots, \mathbf{e}_{i} \times \mathbf{r}_{N}\right)\right\}_{i=1}^{3},
$$


es decir, la componente del vector de velocidad del sistema, $\mathbf{v}$, que rota la configuración $\mathbf{r}$ como un cuerpo rígido, vive en el espacio tangente

$$
T_{\mathbf{r}}(\mathcal{M}(\mathbf{r}))=\operatorname{span}\left\{\mathbf{E}_{i} \times \mathbf{r}\right\}_{i=1}^{3},
$$

donde

$$
\mathcal{M}(\mathbf{r})=\left\{\Omega(\mathbf{r})=\left(\Omega\left(\mathbf{r}_{1}\right), \ldots, \Omega\left(\mathbf{r}_{N}\right)\right): \Omega \in S O(3)\right\}
$$

son todas las rotaciones de una configuración $\mathbf{r}$ vistas como un cuerpo rígido, que al servir como órbita de la acción del grupo de rotaciones $S O(3)$ que actúa sobre $\mathbf{r}, \mathcal{M}(\mathbf{r})$ es una subvariedad de $\left(\mathbb{R}^{3}\right)^{N-1}$. El espacio tangente, $S_{\text {rot }}(\mathbf{r})=T_{\mathbf{r}}(\mathcal{M}(\mathbf{r}))$, define entonces un subespacio de $T_{\mathbf{r}}\left(\mathbb{R}^{3}\right)^{N-1}$ el cual sirve como hogar para las componentes del vector de velocidad del sistema que definen la rotación del cuerpo rígido, además como los tres vectores $\left\{\mathbf{E}_{i} \times \mathbf{r}\right\}_{i=1}^{3}$ son linealmente independientes, éstos forman una base para $S_{r o t}(\mathbf{r})$ por lo tanto la dimensión de $S_{\text {rot }}(\mathbf{r})$ es tres, y lo escribimos como, $\operatorname{dim}\left(S_{\text {rot }}(\mathbf{r})\right)=3$.

Retomando, $W_{1}$, es la proyección de $\mathbf{v}$ al subespacio $S_{\text {rot }}(\mathbf{r})=\operatorname{span}\left\{\mathbf{E}_{i} \times \mathbf{r}\right\}_{i=1}^{3}$, es decir,

$$
\begin{aligned}
W_{1} & =\operatorname{proy}_{S_{r o t}} \mathbf{v} \\
& =\operatorname{proy}_{\mathbf{E}_{1} \times \mathbf{r}} \mathbf{v}+\operatorname{proy}_{\mathbf{E}_{2} \times \mathbf{r}} \mathbf{v}+\operatorname{proy}_{\mathbf{E}_{3} \times \mathbf{r}} \mathbf{v} \\
& =\frac{\left\langle\mathbf{E}_{1} \times \mathbf{r}, \mathbf{v}\right\rangle}{\left|\mathbf{E}_{1} \times \mathbf{r}\right|} \frac{\mathbf{E}_{1} \times \mathbf{r}}{\left|\mathbf{E}_{1} \times \mathbf{r}\right|}+\frac{\left\langle\mathbf{E}_{2} \times \mathbf{r}, \mathbf{v}\right\rangle}{\left|\mathbf{E}_{2} \times \mathbf{r}\right|} \frac{\mathbf{E}_{2} \times \mathbf{r}}{\left|\mathbf{E}_{2} \times \mathbf{r}\right|}+\frac{\left\langle\mathbf{E}_{3} \times \mathbf{r}, \mathbf{v}\right\rangle}{\left|\mathbf{E}_{3} \times \mathbf{r}\right|} \frac{\mathbf{E}_{3} \times \mathbf{r}}{\left|\mathbf{E}_{3} \times \mathbf{r}\right|}=\sum_{i=1}^{3} s_{i} \frac{\mathbf{E}_{i} \times \mathbf{r}}{\left|\mathbf{E}_{i} \times \mathbf{r}\right|},
\end{aligned}
$$

donde $s_{i}$ está relacionado con la $i$-ésima componente del momento angular, puesto que al proyectar $\mathbf{v}$ a lo largo del vector $\mathbf{E}_{i} \times \mathbf{r}$ con $i=1,2,3$ (la figura 2.4 muestra la situación) se tiene que la componente de $\mathbf{v}$ en dirección de $\mathbf{E}_{i} \times \mathbf{r}$ es

$$
s_{i}=\frac{\left\langle\mathbf{E}_{i} \times \mathbf{r}, \mathbf{v}\right\rangle}{\left|\mathbf{E}_{i} \times \mathbf{r}\right|}=\frac{c_{i}}{\left|\mathbf{E}_{i} \times \mathbf{r}\right|},
$$

donde $c_{i}$ es la $i$-ésima componente del momento angular, y es un escalar.

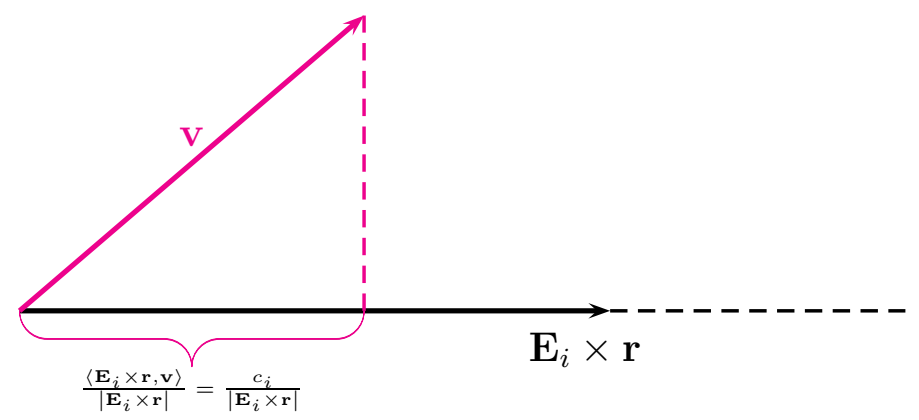

Figura 2.4: Componente de $\mathbf{v}$ a lo largo del vector $\mathbf{E}_{i} \times \mathbf{r}$, con $i=1,2,3$.

Entonces la componente de la velocidad, $W_{1}$, está determinada de manera única por el momento angular $c_{i}=\mathbf{E}_{i} \times \mathbf{r}$ y la base de la ecuación (2.7). De aquí podemos escribir que 
el vector $W_{1}=W_{1}(\mathbf{r}, \mathbf{c})$. Si ahora desarrollamos el último término de la ecuación (2.8) se obtiene que existe un vector $\mathbf{s}=\mathbf{s}(\mathbf{r}, \mathbf{c}) \in \mathbb{R}^{3}$ tal que

$$
W_{1}(\mathbf{r}, \mathbf{c})=\left(\left(s_{1}, s_{2}, s_{3}\right), \ldots,\left(s_{1}, s_{2}, s_{3}\right)\right) \times \mathbf{r}=(\mathbf{s}, \ldots, \mathbf{s}) \times \mathbf{r},
$$

y por lo tanto

$$
W_{1}(\mathbf{r}, \mathbf{c})=\sum_{i=1}^{3} s_{i} \frac{\mathbf{E}_{i} \times \mathbf{r}}{\left|\mathbf{E}_{i} \times \mathbf{r}\right|}=\mathbf{S} \times \mathbf{r}, \quad \text { con } \quad \mathbf{S}=(\mathbf{s}, \ldots, \mathbf{s}) \in\left(\mathbb{R}^{3}\right)^{N-1} .
$$

Ahora, consideremos la siguiente situación, supongamos que el momento angular es cero, por lo cual las partículas no están rotando, en este caso el vector de velocidad rotacional es cero, $W_{1}=0$.

Por otro lado, consideremos que las partículas están restringidas a tener movimiento de rotación en un plano, sin pérdida de generalidad supongamos que ese plano es el $x$ - $y$, en este caso dos componentes del momento angular son nulas, es decir,

$$
\mathbf{c}=\left(\left\langle\mathbf{E}_{1} \times \mathbf{r}, \mathbf{v}\right\rangle,\left\langle\mathbf{E}_{2} \times \mathbf{r}, \mathbf{v}\right\rangle,\left\langle\mathbf{E}_{3} \times \mathbf{r}, \mathbf{v}\right\rangle\right)=\left(0,0, c_{3}\right),
$$

puesto que las partículas se encuentran rotando alrededor de un vector paralelo al eje $z$. Por lo tanto, en este caso se tiene que el vector de velocidad rotacional es la proyección de $\mathbf{v}$ al vector $\mathbf{E}_{3} \times \mathbf{r}$,

$$
W_{1}(\mathbf{r}, \mathbf{c})=\operatorname{proy}_{\mathbf{E}_{3} \times \mathbf{r}} \mathbf{v}=s_{3} \frac{\mathbf{E}_{3} \times \mathbf{r}}{\left|\mathbf{E}_{3} \times \mathbf{r}\right|}, \quad \text { donde } \quad s_{3}=\frac{c_{3}}{\left|\mathbf{E}_{3} \times \mathbf{r}\right|} .
$$

De esta forma cuando tenemos movimientos coplanares el vector $W_{1}$ es más sencillo, puesto que la única proyección de $\mathbf{v}$ distinta de cero, es la proyección al vector $\mathbf{E}_{3} \times \mathbf{r}$. En este caso el subespacio $S_{r o t}$ estará generado únicamente por dicho vector, es decir,

$$
S_{\text {rot }}=\operatorname{span}\left\{\mathbf{E}_{3} \times \mathbf{r}\right\}
$$

entonces el subespacio tendrá dimensión uno, $\operatorname{dim}\left(S_{\text {rot }}\right)=1$, puesto que el sistema estará rotando específicamente en un solo eje de rotación para la configuración.

Hemos definido la componente de la velocidad rotacional, en lo que sigue estudiaremos la segunda componente de la velocidad, dedicada al cambio en el tamaño del sistema.

\section{Velocidad escalar}

La velocidad escalar, $W_{2}$, se encuentra en el complemento ortogonal de $S_{\text {rot }}$ y representa el cambio en el tamaño del sistema, es decir, la forma de la configuración nunca cambia solamente su escala, así la velocidad escalar del sistema está dada por la razón de cambio en el tamaño de $\mathbf{r}$ en la dirección de un vector unitario, matemáticamente esto se expresa como

$$
W_{2}=\left(\frac{d}{d t}|\mathbf{r}|\right) \frac{\mathbf{r}}{|\mathbf{r}|} \text {. }
$$


Luego, de la relación $\mathbf{r} \frac{d}{d t}|\mathbf{r}|=\frac{1}{2} \frac{d}{d t}\langle\mathbf{r}, \mathbf{r}\rangle=\langle\mathbf{r}, \mathbf{v}\rangle$ se sigue que

$$
\frac{d}{d t}|\mathbf{r}|=\frac{1}{|\mathbf{r}|}\langle\mathbf{r}, \mathbf{v}\rangle=\left\langle\frac{\mathbf{r}}{|\mathbf{r}|}, \mathbf{v}\right\rangle
$$

es decir, $\frac{d}{d t}|\mathbf{r}|$ es la componente de $\mathbf{v}$ en dirección del subespacio $S_{\text {scal }}(\mathbf{r})=\left\{t \frac{\mathbf{r}}{|\mathbf{r}|}: t \in \mathbb{R}\right\}, \mathrm{y}$ es un escalar, cuya interpretación geométrica se observa en la figura 2.5.

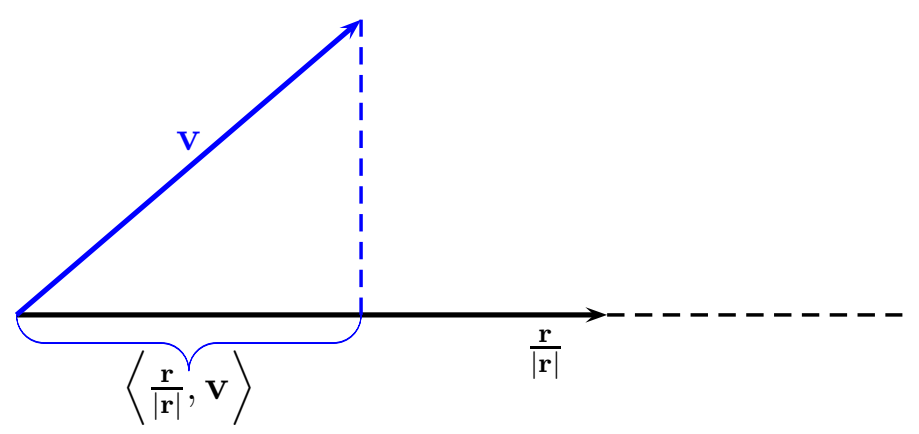

Figura 2.5: Proyección de $\mathbf{v}$ a lo largo del vector unitario $\frac{\mathbf{r}}{|\mathbf{r}|}$.

Por lo tanto, podemos escribir

$$
W_{2}=\left\langle\frac{\mathbf{r}}{|\mathbf{r}|}, \mathbf{v}\right\rangle \frac{\mathbf{r}}{|\mathbf{r}|}=\frac{\langle\mathbf{r}, \mathbf{v}\rangle}{|\mathbf{r}|} \frac{\mathbf{r}}{|\mathbf{r}|}=\operatorname{proy}_{S_{\text {scal }}} \mathbf{v},
$$

la cual, describe el cambio escalar a lo largo de la linea $S_{\text {scal }}(\mathbf{r})$ por lo cual su dimensión claramente es uno, $\operatorname{dim}\left(S_{\text {scal }}\right)=1, \mathrm{y}$ por lo tanto $W_{2}$ siempre vive en un subespacio de dimensión uno.

Como mencionamos anteriormente, el subespacio $S_{\text {scal }}$ se encuentra en el complemento ortogonal de $S_{\text {rot }}$, es decir,

$$
S_{\text {scal }}(\mathbf{r})=\left\{t \frac{\mathbf{r}}{|\mathbf{r}|}: t \in \mathbb{R}\right\} \subseteq\left(S_{\text {rot }}\right)^{\perp},
$$

puesto que, todo elemento de $S_{\text {scal }}$ es ortogonal al conjunto que genera a $S_{\text {rot }}$, esto es,

$$
\left\langle\mathbf{E}_{i} \times \mathbf{r}, t \frac{\mathbf{r}}{|\mathbf{r}|}\right\rangle=\frac{t}{|\mathbf{r}|}\left\langle\mathbf{E}_{i} \times \mathbf{r}, \mathbf{r}\right\rangle=\frac{t}{|\mathbf{r}|}\left\langle\mathbf{E}_{i}, \mathbf{r} \times \mathbf{r}\right\rangle=0
$$

para $i=1,2,3$, y con $t \in \mathbb{R}$.

Otro resultado interesante es el siguiente: sabemos que el momento de inercia se puede escribir en términos del producto escalar $\langle$,$\rangle como I=\langle\mathbf{r}, \mathbf{r}\rangle=|\mathbf{r}|^{2}$ (ver ecuación (2.1)), luego, la razón de cambio con respecto al tiempo del momento de inercia, $\dot{I}=\frac{d I}{d t}$, está dada por

$$
\frac{\dot{I}}{2}=\langle\mathbf{r}, \mathbf{v}\rangle
$$


lo anterior nos permite escribir la ecuación (2.9) como

$$
W_{2}(\mathbf{r}, I)=\frac{\dot{I}}{2 \sqrt{I}} \frac{\mathbf{r}}{|\mathbf{r}|},
$$

por lo tanto, hemos obtiene que la componente de la velocidad, $W_{2}$, está relacionada con la razón de cambio con respecto al tiempo del tamaño del sistema, $\dot{I}$, puesto que el momento de inercia $I$ establece una forma de medir el tamaño del sistema.

Consideremos la situación particular donde el momento de inercia es una constante, en este caso el vector de velocidad escalar es cero, $W_{2}=0$.

En resumen, sabemos cual es el comportamiento de la velocidad rotacional y velocidad escalar, $W_{1}, W_{2}$. La primera relacionada con el momento angular, que es característico de cuerpos con movimiento de rotación; mientras que la segunda se relaciona con el momento de inercia, el cual establece una forma de medir el tamaño del sistema. Nuestro siguiente propósito es dar una expresión para la tercer componente de la velocidad, así como hablar de la dimensión del subespacio que la contiene.

\section{Velocidad configuracional}

La velocidad configuracional, $W_{3}$, dedicada al cambio en la forma de la configuración, es el subespacio final, $S_{\text {conf } f}(\mathbf{r})$, que consiste en esas componentes de la velocidad que cambian (deforman) la configuración $\mathbf{r}$. Matemáticamente, $S_{\text {conf }}(\mathbf{r})$ es el complemento ortogonal en $T_{\mathbf{r}}\left(\mathbb{R}^{3}\right)^{N-1}$ del espacio generado por $S_{\text {rot }}(\mathbf{r})$ y $S_{\text {scal }}(\mathbf{r})$, es decir,

$$
S_{\text {conf }}=\left(S_{\text {rot }} \oplus S_{\text {scal }}\right)^{\perp} .
$$

Una forma de obtener una expresión explícita para este último vector de la descomposición de la velocidad de Saari, $W_{3}$, la tenemos de la ecuación (2.6), es decir, definimos a la velocidad configuracional como

$$
W_{3}=\mathbf{v}-\left(W_{1}+W_{2}\right),
$$

además $W_{3}$ también es tangente a la subvariedad $\mathcal{M}(\mathbf{r})$, y está en términos de la velocidad rotacional y velocidad escalar, las cuales sabemos están relacionadas con el momento angular y el momento de inercia, respectivamente.

De acuerdo con lo que hemos estudiando, se ha descompuesto el espacio tangente, $T_{\mathbf{r}}\left(\mathbb{R}^{3}\right)^{N-1}$, como la suma ortogonal de tres subespacios, $S_{\text {rot }}, S_{\text {scal }}, S_{\text {conf }}$, y puesto que toda suma ortogonal es suma directa podemos escribir

$$
T_{\mathbf{r}}\left(\mathbb{R}^{3}\right)^{N-1}=S_{\text {rot }} \oplus S_{\text {scal }} \oplus S_{\text {conf }},
$$

donde el subespacio $S_{\text {rot }}$ de dimensión tres, es donde vive el vector $W_{1}$, y el subespacio $S_{\text {scal }}$ de dimensión siempre igual a uno, es donde vive el vector $W_{2}$, de aquí es natural preguntarnos cuál es la dimensión del tercer subespacio $S_{\text {conf }}$, en el cual vive el vector $W_{3}$, y puesto que 
los primeros dos subespacios tiene dimensión pequeña esto nos hace esperar que la dimensión del tercero es bastante grande; entonces para encontrar dicha dimensión sabemos

$$
\operatorname{dim}\left(T_{\mathbf{r}}\left(\mathbb{R}^{3}\right)^{N-1}\right)=\operatorname{dim}\left(S_{\text {rot }}\right)+\operatorname{dim}\left(S_{\text {scal }}\right)+\operatorname{dim}\left(S_{\text {conf }}\right),
$$

de aquí,

$$
\operatorname{dim}\left(S_{\text {conf }}\right)=\operatorname{dim}\left(T_{\mathbf{r}}\left(\mathbb{R}^{3}\right)^{N-1}\right)-\left(\operatorname{dim}\left(S_{\text {rot }}\right)+\operatorname{dim}\left(S_{\text {scal }}\right)\right)=3(N-1)-(3+1),
$$

y haciendo el recuento de dimensiones se obtiene que la dimensión del subespacio donde vive $W_{3}$ es

$$
\operatorname{dim}\left(S_{\text {conf }}\right)=3 N-7,
$$

que claramente como lo habíamos esperado, la dimensión del tercer subespacio generalmente será bastante grande a diferencia de los dos primeros.

Luego, en el capítulo uno vimos que la función homogénea de grado cero, $I U^{2}$, mide el cambio en la forma de la configuración, entonces $I U^{2}$ mide a $W_{3}$, por lo que si $I U^{2}=0$ entonces la componente de la velocidad dedicada al cambio en la configuración $W_{3}=0$. Considerar la situación particular $W_{3}=0$, significa que la configuración no cambia, ésta mantiene las mismas clases de equivalencia módulo rotaciones y homotecias.

En esta sección vimos que $W_{1}$ rota la configuración $\mathbf{r}$ como un cuerpo rígido, es decir, cambia la orientación manteniendo la configuración inalterada. Luego, la componente de la velocidad $W_{2}$ cambia el tamaño en la escala de la configuración, entonces $W_{1}$ y $W_{2}$ mantienen la configuración con las mismas clases de equivalencia módulo rotaciones y homotecias, sin embargo, el término $W_{3}$, cambia la forma de la configuración, es decir esta componente de $\mathbf{v}$ obliga a la configuración a cambiar dichas clases de equivalencia.

En conclusión, la velocidad $\mathbf{v}$ se descompuesto ortogonalmente con respecto al producto interior $\langle$,$\rangle como \mathbf{v}=W_{1}+W_{2}+W_{3}$. La primer componente, $W_{1}$ es tangente a la subvariedad $\mathcal{M}(\mathbf{r})$, que representa la totalidad de los movimientos de rotación de las partículas vistas como un cuerpo rígido, es decir, $W_{1}$ es la componente de $\mathbf{v}$ que está en el espacio tangente de la subvariedad de rotaciones del cuerpo rígido, $T_{\mathbf{r}}(\mathcal{M}(\mathbf{r}))$. Para encontrar una representación de $W_{1}$, proyectamos $\mathbf{v}$ sobre $T_{\mathbf{r}}(\mathcal{M}(\mathbf{r}))$ donde la proyección es con respecto al producto interno $\langle$,$\rangle . La segunda componente, W_{2}$ captura las componentes de la velocidad que están cambiando el tamaño de la configuración y la cual está en la dirección del vector unitario $\frac{\mathbf{r}}{|\mathbf{r}|}$. Dado que este vector es ortogonal a la subvariedad $\mathcal{M}(\mathbf{r})$, es obvio que $W_{1}$ y $W_{2}$ son vectores ortogonales. La última componente $W_{3}$ está dada por $W_{3}=\mathbf{v}-\left(W_{1}+W_{2}\right)$. De acuerdo con la construcción, estos tres vectores son ortogonales entre sí. Además, $W_{3}$ es tangente a $\mathcal{M}(\mathbf{r})$, y ésta es la componente de la velocidad que cambia la forma de la configuración.

Por último, si bien sabemos cuales son las relaciones que rigen el comportamiento de las velocidades rotacional y escalar, $W_{1}$ y $W_{2}$ respectivamente, no podemos decir lo mismo acerca de $W_{3}$, ya que no tenemos conocimiento de los parámetros que controlan el cómo cambia la forma de la configuración. Esto sugiere que para conocer más sobre el problema del los $N$-cuerpos, es necesario tener una mejor comprensión de las propiedades y efectos de $W_{3}$. 


\subsection{Descomposición de la velocidad para el problema de los 2-cuerpos}

Una vez estudiada la descomposición de la velocidad de Saari, el primer problema de los $N$-cuerpos que viene a la mente para obtener la descomposición ortogonal del vector de velocidad del sistema, es el problema de los 2-cuerpos, simplemente porque fue el primero en ser estudiado, conocemos todo sobre él y más aún por la gran importancia que tiene en mecánica celeste. En esta sección obtendremos la descomposición de la velocidad del problema de los 2-cuerpos, cuyas ecuaciones de movimiento están dadas por

$$
m_{1} \ddot{\mathbf{r}}_{1}=\frac{m_{1} m_{2}}{\left|\mathbf{r}_{2}-\mathbf{r}_{1}\right|^{3}}\left(\mathbf{r}_{2}-\mathbf{r}_{1}\right), \quad m_{2} \ddot{\mathbf{r}}_{2}=\frac{m_{1} m_{2}}{\left|\mathbf{r}_{1}-\mathbf{r}_{2}\right|^{3}}\left(\mathbf{r}_{1}-\mathbf{r}_{2}\right)
$$

donde la masa $m_{j}$ tiene posición $\mathbf{r}_{j} \in \mathbb{R}^{3}$ y aceleración $\ddot{\mathbf{r}}_{j} \in \mathbb{R}^{3}$ al tiempo $t \in \mathbb{R}$ para $j=1,2$. El centro de masa se encuentra fijo en el origen y estudiamos el movimiento con respecto a éste, además el movimiento tiene lugar en un plano ortogonal al momento angular que pasa por el origen en $\mathbb{R}^{3}$, así el espacio de configuración se reduce a $\mathbb{R}^{2}$.

Puesto que el centro de masa permanece fijo en el origen, se tiene la siguiente relación

$$
m_{1} \mathbf{r}_{1}+m_{2} \mathbf{r}_{2}=0
$$

de la cual se obtiene

$$
\mathbf{r}_{2}=-\frac{m_{1}}{m_{2}} \mathbf{r}_{1}=-a \mathbf{r}_{1}, \quad \text { donde } \quad a=\frac{m_{1}}{m_{2}}
$$

es decir, la posición de la masa $m_{2}$ es un múltiplo escalar de la posición de la masa $m_{1}$ y en dirección opuesta. Sean

$$
\mathbf{r}=\left(\mathbf{r}_{1}, \mathbf{r}_{2}\right)=\left(x_{1}, y_{1}, 0,-a x_{1},-a y_{1}, 0\right) \quad \mathrm{y} \quad \mathbf{v}=\dot{\mathbf{r}}=\left(\dot{\mathbf{r}}_{1}, \dot{\mathbf{r}}_{2}\right)=\left(\dot{x}_{1}, \dot{y}_{1}, 0,-a \dot{x}_{1},-a \dot{y}_{1}, 0\right)
$$

los vectores de configuración y velocidad del sistema de los dos cuerpos, respectivamente.

Además, utilizando la relación (2.12) el sistema (2.11), se escribe como

$$
\ddot{\mathbf{r}}_{1}=-\frac{m_{1}+m_{2}}{\left|\mathbf{r}_{2}-\mathbf{r}_{1}\right|^{3}} \mathbf{r}_{1}, \quad \ddot{\mathbf{r}}_{2}=-\frac{m_{1}+m_{2}}{\left|\mathbf{r}_{1}-\mathbf{r}_{2}\right|^{3}} \mathbf{r}_{2}
$$

es decir, podemos considerar que las partículas se encuentran en configuración central. A continuación iniciamos con el estudio de la descomposición ortogonal del vector de velocidad.

\section{$W_{1}$, Velocidad rotacional}

Para obtener la componente de la velocidad dedicada a rotar las dos partículas como un cuerpo rígido, primero necesitamos encontrar el momento angular para el problema de los dos cuerpos, el cual vimos anteriormente se escribe como

$$
\mathbf{c}=\left(c_{1}, c_{2}, c_{3}\right)=\left(\left\langle\mathbf{E}_{1} \times \mathbf{r}, \mathbf{v}\right\rangle,\left\langle\mathbf{E}_{2} \times \mathbf{r}, \mathbf{v}\right\rangle,\left\langle\mathbf{E}_{3} \times \mathbf{r}, \mathbf{v}\right\rangle\right)
$$


en este caso utilizamos el producto escalar y producto vectorial del sistema con vectores $\mathbf{A}, \mathbf{B}$ $\in \mathbb{R}^{6}$, esto es, $\langle\mathbf{A}, \mathbf{B}\rangle=m_{1}\left(\mathbf{a}_{1}, \mathbf{b}_{1}\right)+m_{2}\left(\mathbf{a}_{2}, \mathbf{b}_{2}\right)$ y $\mathbf{A} \times \mathbf{B}=\left(\mathbf{a}_{1} \times \mathbf{b}_{1}, \mathbf{a}_{2} \times \mathbf{b}_{2}\right)$, donde $\left(\mathbf{a}_{j}, \mathbf{b}_{j}\right)$, $\mathbf{a}_{j} \times \mathbf{b}_{j}$ son el producto escalar y vectorial usual en $\mathbb{R}^{3}$, respectivamente, para $j=1,2$.

Por lo cual, las tres componentes del momento angular son:

$$
\begin{aligned}
& c_{1}=\left\langle\left(0,0, y_{1}, 0,0,-a y_{1}\right),\left(\dot{x}_{1}, \dot{y}_{1}, 0,-a \dot{x}_{1},-a \dot{y}_{1}, 0\right)\right\rangle=0, \\
& c_{2}=\left\langle\left(0,0,-x_{1}, 0,0, a x_{1}\right),\left(\dot{x}_{1}, \dot{y}_{1}, 0,-a \dot{x}_{1},-a \dot{y}_{1}, 0\right)\right\rangle=0, \\
& c_{3}=\left\langle\left(-y_{1}, x_{1}, 0, a y_{1},-a x_{1}, 0\right),\left(\dot{x}_{1}, \dot{y}_{1}, 0,-a \dot{x}_{1},-a \dot{y}_{1}, 0\right)\right\rangle=m_{1}(1+a)\left(x_{1} \dot{y}_{1}-y_{1} \dot{x}_{1}\right),
\end{aligned}
$$

como se puede observar, el momento angular es un vector ortogonal al plano donde se da el movimiento de las dos partículas, $\mathbf{c}=\left(0,0, c_{3}\right)$, y la componente de la velocidad que rota a las dos partículas como un cuerpo rígido, es la proyección de $\mathbf{v}$ al subespacio $S_{\text {rot }}$ el cual tiene dimensión uno,

$$
S_{\text {rot }}(\mathbf{r})=\operatorname{span}\left\{\mathbf{E}_{3} \times \mathbf{r}\right\}
$$

entonces $W_{1}$ es la proyección de $\mathbf{v}$ al vector $\mathbf{E}_{3} \times \mathbf{r}$, es decir,

$$
W_{1}=\operatorname{proy}_{\mathbf{E}_{3} \times \mathbf{r}} \mathbf{v}=\frac{\left\langle\mathbf{E}_{3} \times \mathbf{r}, \mathbf{v}\right\rangle}{\left|\mathbf{E}_{3} \times \mathbf{r}\right|} \frac{\mathbf{E}_{3} \times \mathbf{r}}{\left|\mathbf{E}_{3} \times \mathbf{r}\right|},
$$

en este caso la velocidad rotacional, $W_{1}$, está determinada de manera única por la tercer componente del momento angular, $c_{3}$, en dirección del vector $\mathbf{E}_{3} \times \mathbf{r}$, es decir, tiene la siguiente expresión

$$
W_{1}=\frac{c_{3}}{\left|\mathbf{E}_{3} \times \mathbf{r}\right|} \frac{\mathbf{E}_{3} \times \mathbf{r}}{\left|\mathbf{E}_{3} \times \mathbf{r}\right|}=\frac{c_{3}}{m_{1}(1+a)\left(x_{1}^{2}+y_{1}^{2}\right)}\left(-y_{1}, x_{1}, 0, a y_{1},-a x_{1}, 0\right),
$$

donde $c_{3}=\left\langle\mathbf{E}_{3} \times \mathbf{r}, \mathbf{v}\right\rangle, \mathbf{E}_{3} \times \mathbf{r}=\left(-y_{1}, x_{1}, 0, a y_{1},-a x_{1}, 0\right),\left|\mathbf{E}_{3} \times \mathbf{r}\right|=\sqrt{m_{1}(1+a)\left(x_{1}^{2}+y_{1}^{2}\right)}$ y $a=\frac{m_{1}}{m_{2}}$.

Ahora, para tener una idea geométrica de lo que sucede con la componente $W_{1}$ del vector de velocidad para problema de los dos cuerpos, sean

$$
W_{1, \mathbf{v}_{1}}=k\left(-y_{1}, x_{1}, 0\right) \text {, }
$$

la parte que le corresponde de $W_{1}$ al vector de velocidad de la partícula con masa $m_{1}, \mathrm{y}$

$$
W_{1, \mathbf{v}_{2}}=-a k\left(-y_{1}, x_{1}, 0\right)=-a W_{1, \mathbf{v}_{1}}
$$

la parte que le corresponde de $W_{1}$ al vector de velocidad de la partícula con masa $m_{2}$, donde $k=\frac{c_{3}}{m_{1}(1+a)\left(x_{1}^{2}+y_{1}^{2}\right)}$.

En realidad, sólo estamos separando en dos el vector dado por la ecuación (2.13). En efecto, $W_{1, \mathbf{v}_{1}}$ y $W_{1, \mathbf{v}_{2}}$, hacen girar a las dos partículas como un cuerpo rígido, puesto que como se puede ver en la figura 2.6 (a), $W_{1, \mathbf{v}_{2}}$ tiene dirección opuesta $W_{1, \mathbf{v}_{1}}$, y éstos hacen girar a las dos partículas como un cuerpo rígido. 


\section{$W_{2}$, Velocidad escalar}

Por definición, la componente de la velocidad dedicada a cambiar el tamaño a la configuración de las dos partículas, esta dada como sigue

$$
W_{2}(\mathbf{r}, I)=\frac{\dot{I}}{2 \sqrt{I}} \frac{\mathbf{r}}{|\mathbf{r}|}
$$

este vector vive en el subespacio $S_{\text {scal }}(\mathbf{r})=\left\{t \frac{\mathbf{r}}{|\mathbf{r}|}: t \in \mathbb{R}\right\}$, cuya dimensión siempre igual a uno. Luego, el subespacio $S_{\text {scal }}$ es el complemento ortogonal de $S_{\text {rot }}$, es decir,

$$
S_{\text {scal }}(\mathbf{r})=\left\{t \frac{\mathbf{r}}{|\mathbf{r}|}: t \in \mathbb{R}\right\}=\left(S_{\text {rot }}\right)^{\perp},
$$

puesto que, todo elemento de $S_{\text {scal }}$ es ortogonal al conjunto que genera a $S_{\text {rot }}$, esto es,

$$
\left\langle\mathbf{E}_{3} \times \mathbf{r}, t \frac{\mathbf{r}}{|\mathbf{r}|}\right\rangle=\frac{t}{|\mathbf{r}|}\left\langle\mathbf{E}_{3}, \mathbf{r} \times \mathbf{r}\right\rangle=0 .
$$

El momento de inercia es: $I=\langle\mathbf{r}, \mathbf{r}\rangle=|\mathbf{r}|^{2}=m_{1}(1+a)\left(x_{1}^{2}+y_{1}^{2}\right)$, luego, la razón de cambio con respecto al tiempo del momento de inercia resulta ser $\dot{I} / 2=m_{1}(1+a)\left(x_{1} \dot{x}_{1}+y_{1} \dot{y}_{1}\right)$, sustituyendo lo anterior en la ecuación (2.14), la componente de la velocidad que cambia la escala de la configuración formada por los dos cuerpos es

$$
W_{2}(\mathbf{r}, I)=\frac{x_{1} \dot{x}_{1}+y_{1} \dot{y}_{1}}{x_{1}^{2}+y_{1}^{2}}\left(x_{1}, y_{1}, 0,-a x_{1},-a y_{1}, 0\right)=\alpha\left(x_{1}, y_{1}, 0,-a x_{1},-a y_{1}, 0\right),
$$

con $\alpha=\frac{x_{1} \dot{x}_{1}+y_{1} \dot{y}_{1}}{x_{1}^{2}+y_{1}^{2}}$ y $a=\frac{m_{1}}{m_{2}}$.

Nuevamente, para tener una idea geométrica del efecto de la componente $W_{2}$, separamos el vector dado por la ecuación (2.15) como

$$
W_{2, \mathbf{v}_{1}}=\alpha\left(x_{1}, y_{1}, 0\right) \quad \text { y } \quad W_{2, \mathbf{v}_{2}}=-a \alpha\left(x_{1}, y_{1}, 0\right)=-a W_{2, \mathbf{v}_{1}},
$$

donde $W_{2, \mathbf{v}_{1}}$ es la parte que le corresponde de $W_{2}$ al vector de velocidad de la partícula con masa $m_{1}$, y $W_{2, \mathbf{v}_{2}}$ la parte que le corresponde de $W_{2}$ al vector de velocidad de la partícula con masa $m_{2}$, respectivamente. Como se observa en la figura 2.6 (b), éstas cambian la escala de la configuración colineal formada por los dos cuerpos.

Hemos obtenido los vectores $W_{1}$ y $W_{2}$ para el problema de los dos cuerpos, resta saber quien es el vector $W_{3}$, dedicado al cambio en la forma de la configuración formada por los dos cuerpos, y el cual vive en el subespacio final $S_{\text {conf }}(\mathbf{r})$, sin embargo, es fácil ver que la dimensión de este subespacio es cero, es decir, $W_{3}=0$, puesto que ya hemos cubierto el espacio tangente, con los dos subespacios ortogonales $S_{\text {rot }}$ y $S_{\text {scal }}$. Así, el vector de velocidad del problema de los dos cuerpos se descompuso como

$$
\mathbf{v}=W_{1}+W_{2},
$$


entonces en este caso, únicamente hay cambios en la orientación de las dos partículas al rotarlas como un cuerpo rígido, o se presentan movimientos de dilatación o contracción preservando al mismo tiempo la configuración colineal de las dos partículas.

Por último, realizamos la descomposición ortogonal del vector de velocidad de problema de los dos cuerpos, esto es, descompusimos el vector de velocidad (véase la figura 2.7) de tal forma que esta descomposición nos permitió conocer cómo influye (afecta) la velocidad en la orientación de las dos partículas vistas como un cuerpo rígido, $W_{1}$, y cómo cambia la escala de la configuración, $W_{2}$, donde ambas componente son ortogonales.

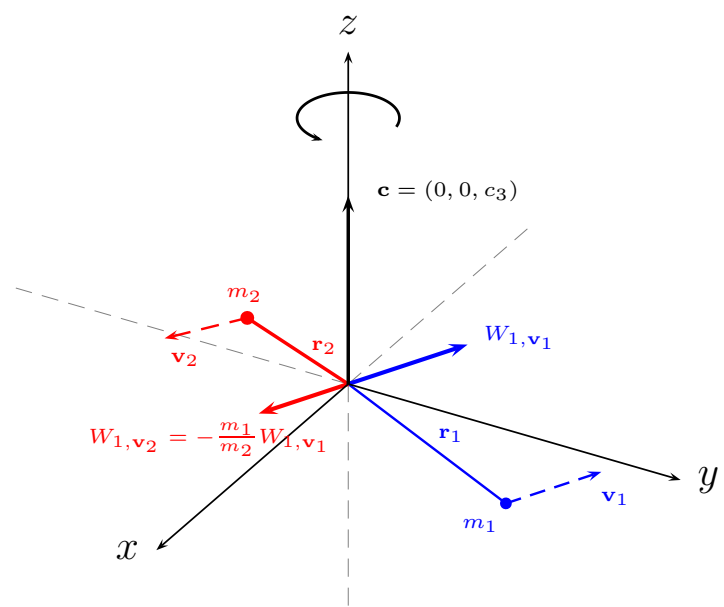

(a) Interpretación geométrica de $W_{1}$ para el problema de los 2-cuerpos.

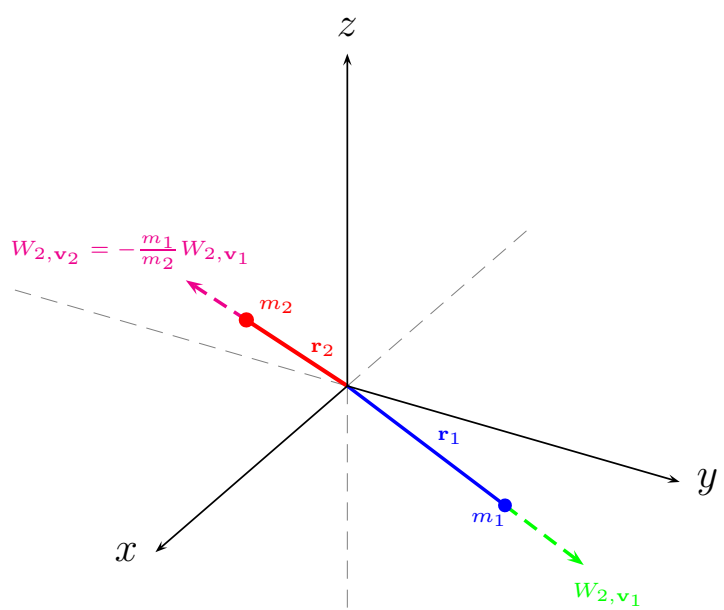

(b) Interpretación geométrica de $W_{2}$ para el problema de los 2-cuerpos.

Figura 2.6: Componentes de la velocidad para el problema de los 2-cuerpos.

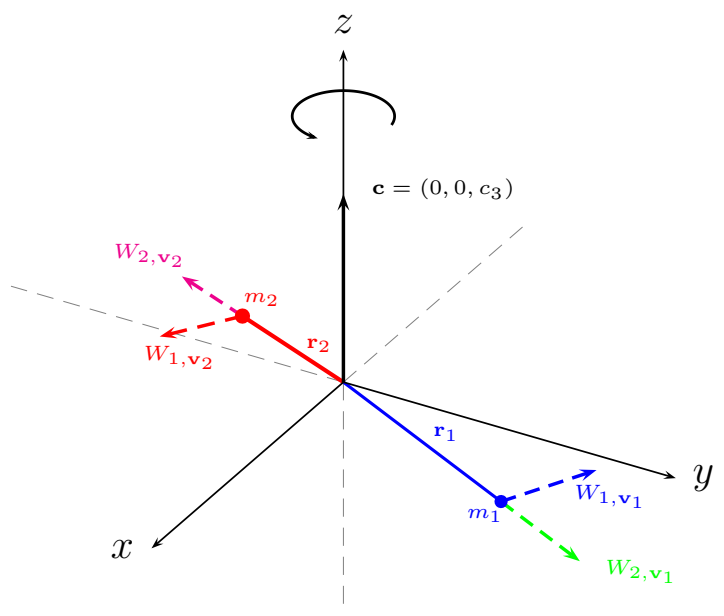

Figura 2.7: Descomposición ortogonal del vector de velocidad del problema de los 2-cuerpos. 


\section{CAPÍTULO 3}

\section{Nueva clasificación para problemas de los $N$-cuerpos}

Sabemos que el problema de los $N$-cuerpos está clasificado de acuerdo al número de partículas puntuales en el espacio euclidiano $\mathbb{R}^{3}$ que se mueven bajo la ley gravitacional de Newton, es decir, es común escuchar hablar del problema de los 2-cuerpos, el problema de los 3-cuerpos, el problema de los 4-cuerpos; etcétera. En este capítulo daremos una nueva forma de clasificar el problema clásico de los $N$-cuerpos, utilizando el vector de velocidad del sistema $\mathbf{v}$, esta nueva clasificación se concentra en la dinámica de las partículas, y no en el número de partículas que se estén estudiando; y para poder realizar esta nueva clasificación haremos uso como es de esperar, de las tres componentes de la velocidad propuestas por Saari.

Es decir, uno de los objetivos de este trabajo es proponer una nueva clasificación para el problema de los $N$-cuerpos, en términos de la descomposición de la velocidad de Saari, por lo cual utilizaremos las tres componentes de la velocidad, $W_{1}, W_{2}$ y $W_{3}$, que como ya estudiamos están dedicadas a determinar cambios en la orientación de la configuración, cambios en el tamaño escalar de la configuración y cambios en la forma de la configuración, respectivamente.

\subsection{Problema de los $N$-cuerpos cero-dimensional}

Para iniciar con el estudio de la nueva clasificación para el problema de los $N$-cuerpos, partimos del caso más sencillo, supongamos que la tercer componente de la velocidad $W_{3}=0$, es decir, vamos a estudiar lo que ocurre con una solución, cuando no cambia la forma de la configuración, [12], [13] y [14].

Definición 3.1 Decimos que un problema de los $N$-cuerpos es cero-dimensional si el vector

$$
W_{3}=0 \text {. }
$$


Entonces cuando $W_{3}=0$, el vector de velocidad del sistema se descompone únicamente como la suma de las dos primeras componentes de la velocidad, es decir,

$$
\mathbf{v}=W_{1}+W_{2},
$$

lo cual nos permite considerar dos importantes casos particulares:

(a) Si $W_{1} \neq 0$ y $W_{2}=0$, es decir, si la primer componente de la velocidad es distinta de cero lo cual significa que la configuración sufre cambios en la orientación al rotar las partículas como un cuerpo rígido; luego la segunda componente igual a cero lo cual indica que el tamaño del sistema no cambia, ya que no hay movimientos de dilatación o contracción preservando al mismo tiempo la configuración. Por lo tanto, en este caso se obtienen las soluciones en equilibrio relativo, caracterizadas porque el sistema rota al rededor del centro de masas como si se tratara de un cuerpo rígido, las distancias mutuas no cambian cuando el tiempo $t$ varia, cada partícula se mueve exactamente en una órbita idéntica, pero sin cambiar el tamaño del sistema.

(b) Si $W_{1}=0$ y $W_{2} \neq 0$, esto es, si ahora la primer componente de la velocidad es cero lo cual quiere decir que la configuración no sufre cambios en la orientación ya que las partículas no presentan rotaciones como cuerpo rígido; luego la segunda componente distinta de cero indica que el tamaño (escala) del sistema cambia, puesto que hay movimientos homotéticos preservando la configuración. Por lo cual, en este caso se tienen las soluciones homotéticas, aquí cada partícula está restringida a permanecer sobre una línea recta que pasa por centro de masa del sistema. Esto significa que las soluciones homotéticas, permiten que las partículas cambien el tamaño en la escala preservando al mismo tiempo la configuración, pero sin rotación de ningún tipo.

Por lo tanto, si $W_{1} \neq 0$ y $W_{2} \neq 0$, es decir, la configuración sufre cambios en la orientación y su tamaño está cambiando. Entonces, tenemos las soluciones homográficas caracterizadas por la existencia de una matriz de rotación $\Omega(t) \in S O(3)$ y una función escalar $R(t)>0$ tal que para cada $j$ y para todo tiempo $t$, se tiene

$$
\mathbf{r}_{j}(t)=R(t) \Omega(t) \mathbf{r}_{j}\left(t_{0}\right),
$$

donde $t_{0}$ es un momento fijo en el tiempo y $\mathbf{r}_{j}\left(t_{0}\right)$ es una configuración central.

Otro caso, es considerar ambas componentes iguales a cero, es decir, $W_{1}=0, W_{2}=0$, y por lo tanto naturalmente se tiene un problema trivial. Entonces la definición 3.1 nos dice que cuando $W_{3}=0$, la solución mantiene la misma configuración central para todo tiempo, esto es, se sabe que las configuraciones centrales se mantienen durante toda la evolución del sistema.

Recordemos que Euler y Lagrange fueron quienes encontraron las primeras soluciones del problema de los tres cuerpos, la configuración inicial de la cual parten tanto Euler como Lagrange es una configuración central. Euler demuestra que si tres masas arbitrarias son colocadas inicialmente en línea recta, de tal forma que la razón de sus distancias satisfaga una fórmula que únicamente depende del valor de las masas, y si además, las velocidades 
iniciales son escogidas adecuadamente, entonces cada partícula se moverá periódicamente sobre una elipse, pero en todo momento las tres partículas se mantendrán sobre una línea recta, conservando siempre la misma razón entre sus distancias. Es decir, encuentra explícitamente una familia completa de órbitas periódicas, las cuales conocemos como soluciones en equilibrio relativo. Aquí las distancias entre los tres cuerpos permanecen fijas por lo que las tres partículas giran sin que haya cambio en la escala. Esto es, el sistema rota al rededor del centro de masas como si se tratara de un cuerpo rígido, las distancias mutuas no cambian cuando el tiempo $t$ varia, cada partícula se mueve exactamente en una órbita idéntica.

Posteriormente, Lagrange encuentra una nueva familia de órbitas periódicas, obtenidas al colocar tres masas arbitrarias sobre los vértices de un triángulo equilátero, donde hay que escoger adecuadamente las velocidades iniciales. Aquí nuevamente cada partícula se moverá sobre una órbita elíptica; en todo momento la configuración será de triángulo equilátero, el cual podrá variar de tamaño pero nunca de forma, es decir, las tres partículas cambian el tamaño en la escala preservando al mismo tiempo la configuración, obteniendo una solución homográfica. Por lo tanto las soluciones encontradas por Euler y Lagrange, son ejemplos de soluciones homográficas, y fueron las primeras soluciones del problema de los tres cuerpos, en cierto sentido son las órbitas naturales más fáciles de describir, lo cual justifica nuestra definición de problema cero-dimensional. Las soluciones homográficas son las únicas soluciones del problema de los $N$-cuerpos que son conocidas explícitamente.

Por lo anterior, ejemplos de sistemas cero-dimensional son los estudiados por Euler y Lagrange, donde las soluciones mantiene la misma configuración central para todo tiempo. Otro ejemplo de sistema cero-dimensional es el problema de los dos cuerpos, como vimos en el capítulo anterior, la descomposición de la velocidad para el problema de los dos cuerpos es de la forma $\mathbf{v}=W_{1}+W_{2}$, es decir, el vector $W_{3}=0$, entonces las soluciones mantienen la misma configuración central durante toda la evolución del sistema. En conclusión, lo que sucede en la situación $W_{3}=0$, es que la solución homográfica puede rotar y cambiar el tamaño de la configuración, pero no su forma.

\subsection{Problema de los $N$-cuerpos uno-dimensional}

Con el propósito de continuar con la clasificación de problemas de los $N$-cuerpos en términos de la descomposición de la velocidad, y como una aportación de este trabajo, damos una segunda definición para sistemas que no son cero-dimensional, para esto ahora necesitamos suponer que el vector $W_{3} \neq 0$, es decir, el sistema original siempre presenta cambio en la forma de la configuración.

Antes de dar nuestra definición es necesario mencionar que el centro de masa juega un papel importante en ella, ya que propondremos dividir las partículas en dos cúmulos, tal que en uno de éstos se encuentre el centro de masa (visto como punto fijo), el cual junto con las partículas que se encuentren en el mismo cúmulo, determinarán si el vector $W_{3}$ para este cúmulo (independiente del segundo) es nulo, es decir, la configuración formada por las partículas y el centro de masa determinaran si cambia la forma de la configuración del cúmulo donde se encuentran. 
Una vez aclarada la importancia que tiene el centro de masa, entonces procedemos a dar nuestra definición de la siguiente manera.

Definición 3.2 Decimos que un problema de los $N$-cuerpos es uno-dimensional si $W_{3} \neq 0$ y las partículas se pueden dividir en dos cúmulos los cuales denotamos por $C_{1}$ y $C_{2}$ tal que el centro de masa se encuentra en alguno de estos cúmulos y $W_{3, C_{1}}=0, W_{3, C_{2}}=0$.

Observemos que $W_{3} \neq W_{3, C_{1}}+W_{3, C_{2}}$. Entonces, la definición de sistemas uno-dimensional dice que el vector $W_{3}$ del sistema debe ser distinto de cero, esto es, el sistema original siempre presenta cambio en la forma de su configuración. Después, dividimos el sistema original en dos cúmulos tal que en uno de estos se encuentre el centro de masa, al analizar los cúmulos de forma independiente se debe cumplir que $W_{3, C_{1}}=0$. Es decir, las partículas del sistema que se encuentren en el primer cúmulo estarán formando alguna configuración, entonces la forma de dicha configuración se debe preservar en ese cúmulo para todo tiempo $t$, así diremos que en el primer cúmulo $W_{3, C_{1}}=0$. Luego, en el segundo cúmulo se deben encontrar el centro de masa y el resto de las partículas, los cuales también estarán formando alguna configuración (independiente de la configuración formada por las otras partículas en el primer cúmulo), y esta configuración en el segundo cúmulo también se debe preservar durante toda la evolución del sistema, por lo cual de igual forma decimos que $W_{3, C_{2}}=0$ en el segundo cúmulo, y por lo tanto tendremos un problema de los $N$-cuerpos cero-dimensional en cada cúmulo.

A estas alturas, el lector podría haberse preguntado cuál es la importancia o lo innovador de esta nueva clasificación, y la respuesta es: clasificar los problemas de $\operatorname{los} N$ cuerpos, en sistemas cero-dimensional o sistemas uno-dimensional, nos permite conocer cómo se comportarán las soluciones. Sin embargo, reconocemos que dicha clasificación se queda corta, en el sentido de que si pudiéramos definir los sistemas dos-dimensional, tal vez podríamos conocer más acerca del problema de los 3-cuerpos, pero aún no sabemos o no tenemos idea de como definir los sistemas dos-dimensional, pero sí sabemos que es un reto que requiere de mucho trabajo a futuro.

A continuación presentamos tres ejemplos de problemas de los $N$-cuerpos, que cumplen ser sistemas uno-dimensional, los cuales son: el problema romboidal generalizado en rotación, el problema isósceles en rotación y el problema colineal de 3-cuerpos en el plano.

\subsubsection{Ejemplo 1: Problema romboidal generalizado en rotación}

Consideramos $N$-partículas puntuales con masa $m_{1}$ localizados en los vértices de un $N$ polígono regular centrado en el origen del plano $x-y$, y dos partículas puntuales, $N+1 \mathrm{y}$ $N+2$ situadas sobre el eje $z$, con masas $m_{2}$, y que están simétricamente localizadas con respecto al origen, por lo cual este es un problema de $N+2$-cuerpos. Véase la figura 3.1.

Supongamos que las partículas de masa $m_{1}$ están rotando alrededor del eje $z$ con velocidad angular constante. Sea $\rho$ la distancia de cada una de estas partículas al origen, entonces la posición de la $j$-ésima partícula es

$$
\mathbf{r}_{j}=\rho \exp \left(j \frac{2 \pi}{N}+\theta\right) i, \quad j=1, \ldots, N
$$


donde $e^{\theta i} \in S^{1}$ representa la rotación al rededor del eje $z$, e $i$ viene de la notación compleja; con el propósito de simplificar la notación escribimos

$$
\mathbf{r}_{j}=\rho\left(x_{j}, y_{j}, 0\right) \quad j=1, \ldots, N,
$$

donde $\left(x_{j}, y_{j}\right)$ es el correspondiente vector unitario. Como es usual el origen lo llevamos al centro de masa, $\mathbf{c}_{m}=\left(\left\langle\mathbf{r}, \mathbf{E}_{1}\right\rangle,\left\langle\mathbf{r}, \mathbf{E}_{2}\right\rangle,\left\langle\mathbf{r}, \mathbf{E}_{3}\right\rangle\right)$.

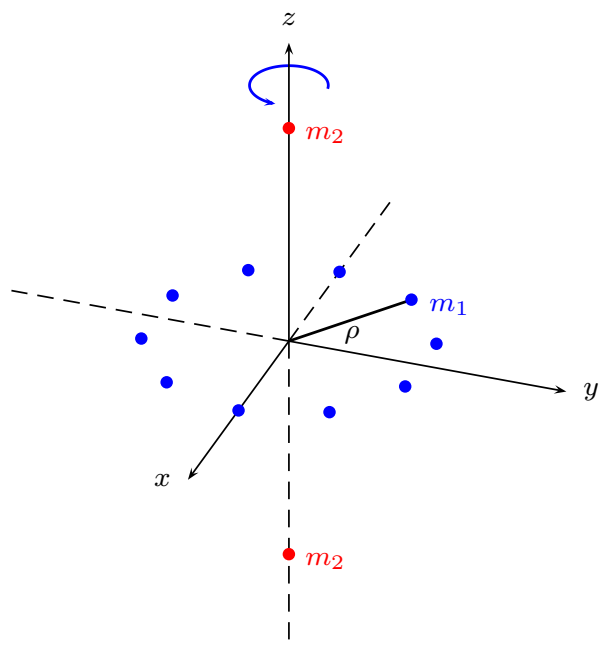

Figura 3.1: Problema romboidal generalizado en rotación.

El problema romboidal generalizado en rotación es un sistema uno-dimensional, en primer lugar se cumple que el vector $W_{3} \neq 0$, ya que la configuración romboidal original sufre deformación conforme evoluciona el sistema.

Luego, podemos dividir las partículas en los siguientes dos cúmulos: el primer cúmulo $C_{1}$, lo pensamos como el que contiene las $N$-partículas situadas en el plano $x-y$, y aquí la forma de la configuración no cambia, ya que recordemos que cualquier $N$-polígono regular con masas iguales colocadas en cada uno de sus vértices forman una configuración central; donde dos posibles movimientos de las partículas son contracciones o expansiones, por supuesto también puede haber rotaciones con combinación de ambos movimientos; por lo tanto en este primer cúmulo $W_{3, C_{1}}=0$. Después, el segundo cúmulo $C_{2}$, lo conforman el centro de masa fijo en el origen junto con las dos partículas restantes $N+1, N+2$, y puesto que estas últimas están localizadas simétricamente respecto al origen sobre el eje $z$, entonces tampoco cambia la forma de la configuración en este segundo cúmulo, por lo cual también $W_{3, C_{2}}=0$; y por lo tanto tenemos un problema cero-dimensional en cada cúmulo, implicando que el problema romboidal generalizado en rotación sea un sistema uno-dimensional.

\subsubsection{Ejemplo 2: Problema isósceles en rotación}

En este caso, consideremos dos partículas de masa $m_{1}$ ubicadas sobre el plano $x-y$ las cuales se encuentran simétricamente localizadas respecto al origen y están rotando al rededor del eje $z$ con una velocidad angular constante. Supongamos que la tercer partícula tiene masa $m_{2}$ y 
se encuentra localizada sobre el eje $z$ a una distancia con respecto al origen de $\sqrt{\frac{M}{2 m_{1}}}$ donde $M$ es la masa total del sistema $M=2 m_{1}+m_{2}$. Una interpretación geométrica se observa en la figura 3.2.

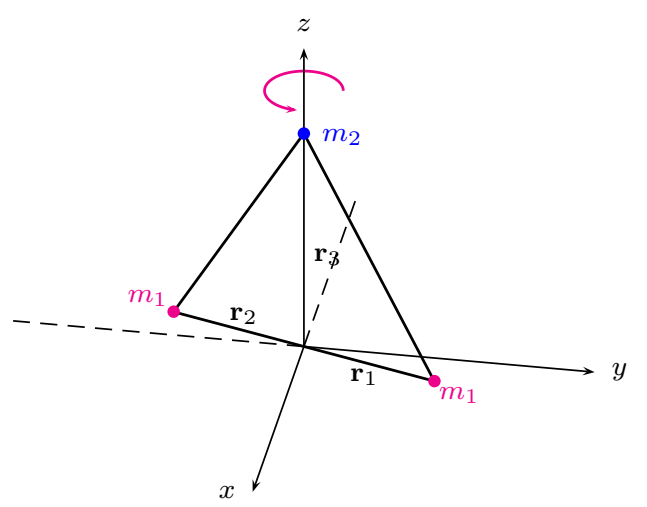

Figura 3.2: Problema isósceles en rotación.

Hasta aquí podría pensarse que el problema isósceles en rotación es similar al romboidal generalizado en rotación, sin embargo, existe una diferencia importante entre éstos, puesto que el centro de masa del problema isósceles en rotación se mueve siempre sobre el eje $z$, es decir,

$$
\mathbf{c}_{m}=\left(\left\langle\mathbf{r}, \mathbf{E}_{1}\right\rangle,\left\langle\mathbf{r}, \mathbf{E}_{2}\right\rangle,\left\langle\mathbf{r}, \mathbf{E}_{3}\right\rangle\right)=m_{2}\left(0,0, z \sqrt{\frac{M}{2 m_{1}}}\right),
$$

y como es usual, lo fijamos en el origen.

El problema isósceles en rotación también es un sistema uno-dimensional, puesto que el vector $W_{3} \neq 0$, ya que la configuración en forma de isósceles presenta cambio conforme avanza el tiempo, cuando la partícula de masa $m_{2}$ se aleja demasiado. Luego, podemos dividir las partículas en los siguientes dos cúmulos: en el primer cúmulo $C_{1}$, se encuentran las partículas de masa $m_{1}$ ubicadas sobre el plano $x-y$, las cuales están simétricamente localizadas con respecto al origen y por lo cual en este cúmulo $W_{3, C_{1}}=0$. El segundo cúmulo, es el conformado por el centro de masa y la partícula de masa $m_{2}$ localizada sobre el eje $z$ a una distancia con respecto al origen de $\sqrt{\frac{M}{2 m_{1}}}$, por lo cual no cambiara la forma de la configuración colineal entre ellos, implicando que también $W_{3, C_{2}}=0$ en este segundo cúmulo. Por lo tanto tenemos un problema cero-dimensional en cada cúmulo $C_{1}$ y $C_{2}$, lo cual implica que el problema isósceles en rotación es un sistema uno-dimensional.

\subsubsection{Ejemplo 3: Problema colineal de 3-cuerpos en el plano}

Por último, el problema colineal de 3-cuerpos en el plano también es un sistema unodimensional. Consideremos tres partículas en las posiciones $\mathbf{r}_{1}, \mathbf{r}_{2}$ y $\mathbf{r}_{3}$ con masas distintas $m_{1}, m_{2}$ y $m_{3}$, respectivamente, las cuales se encuentran colocadas de tal forma que siempre están sobre una linea recta en el plano $x-y$. Como es usual llevamos el origen al centro de masa. Además, supongamos que las tres partículas están rotando, es decir, su rotación será 
preservando la configuración colineal sobre el plano $x-y$, por lo cual la recta sobre la que se encuentren estará cambiando conforme roten. La interpretación geométrica se muestra en la figura 3.3 (a).

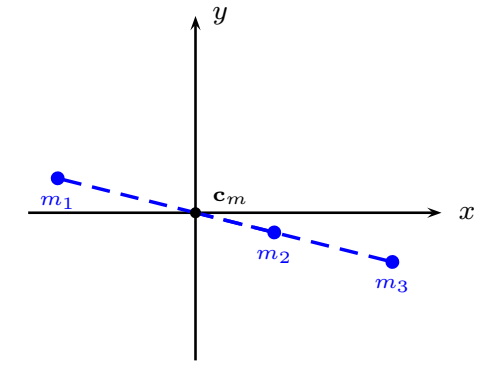

(a) Tres partículas colineales en el plano $x-y$.

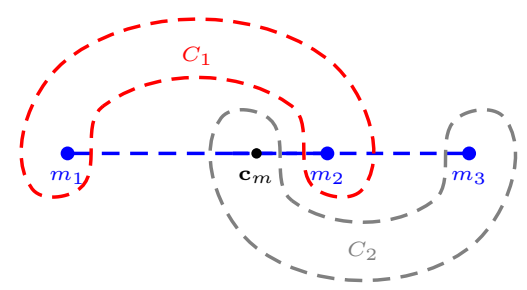

(b) Cúmulos $C_{1}$ y $C_{2}$.

Figura 3.3: Problema Colineal de 3-cuerpos en el plano.

De acuerdo a la definición 3.2, el problema colineal de tres cuerpos en el plano es un sistema uno-dimensional, puesto que el vector $W_{3}$ es distinto de cero, ya que el sistema original siempre presenta cambio en la forma de su configuración, por ejemplo la partícula con masa $m_{1}$, se puede acercar demasiado al centro de masa, $\mathbf{c}_{m}$, provocando que haya cambio en la configuración colineal original de los 3-cuerpos.

Luego, podemos dividir las partículas en dos cúmulos como se observa en la figura 3.3 (b), en el cúmulo $C_{1}$ se encuentran las partículas con masas $m_{1}$ y $m_{2}$, aquí $W_{3, C_{1}}=0$, es decir, la forma de la configuración colineal formada por $m_{1}$ y $m_{2}$ se preservará en ese cúmulo para todo tiempo $t$. Para el cúmulo $C_{2}$, de igual forma se tiene $W_{3, C_{2}}=0$, puesto que en este cúmulo el centro de masa, $\mathbf{c}_{m}$, junto con la partícula que resta, $m_{3}$, forman una configuración colineal de 2-cuerpos, la cual se preservará durante toda la evolución del sistema. Por lo tanto, tenemos un problema cero-dimensional en cada cúmulo, $C_{1}$ y $C_{2}$, lo cual quiere decir que el problema colineal de tres cuerpos en plano, es un sistema uno-dimensional.

En conclusión, en este tercer capítulo hemos utilizado la descomposición de la velocidad de Saari para dar una nueva clasificación de problemas de los $N$-cuerpos, la cual se basa en la dinámica de las partículas y nos permite conocer cómo se comportarán las soluciones, además dimos ejemplos de sistemas cero-dimensional y sistemas uno-dimensional. 


\section{CAPÍTULO 4}

\section{Coordenadas para obtener las componentes de la velocidad}

En este capítulo obtendremos las tres componentes de la descomposición de la velocidad, para cada uno de los sistemas uno-dimensionales estudiados en el capítulo anterior. Para ello daremos diferentes sistemas coordenados, con el propósito de encontrar una representación de las tres componentes de la descomposición de la velocidad.

\subsection{Componentes de la velocidad: Problema romboidal generalizado en rotación}

Recordemos el problema romboidal generalizado en rotación, esto es, tenemos $N$ partículas puntuales con masa $m_{1}$ localizados en los vértices de un $N$-polígono regular centrado en el origen del plano $x-y$, y dos partículas puntuales, $N+1$ y $N+2$ situadas sobre el eje $z$, con masas $m_{2}$, las cuales están simétricamente localizadas con respecto al origen. Sea $\rho$ la distancia de cada una de estas partículas al origen, entonces la posición de la $j$-ésima partícula es

$$
\mathbf{r}_{j}=\rho \exp \left(j \frac{2 \pi}{N}+\theta\right) i, \quad j=1,2, \ldots, N,
$$

donde $e^{\theta i} \in S^{1}$ representa la rotación al rededor del eje $z$, e $i$ viene de la notación compleja; con el propósito de simplificar la notación escribimos

$$
\mathbf{r}_{j}=\rho\left(x_{j}, y_{j}, 0\right) \quad j=1, \ldots, N,
$$

donde $\left(x_{j}, y_{j}\right)$ es el correspondiente vector unitario (véase la figura 3.1). En este caso el vector de configuración es

$$
\begin{aligned}
\mathbf{r} & =\left(\mathbf{r}_{1}, \mathbf{r}_{2}, \ldots, \mathbf{r}_{N-1}, \mathbf{r}_{N}, \mathbf{r}_{N+1}, \mathbf{r}_{N+2}\right) \\
& =\left(\rho\left(x_{1}, y_{1}, 0\right), \rho\left(-x_{1},-y_{1}, 0\right), \ldots, \rho\left(x_{N}, y_{N}, 0\right), \rho\left(-x_{N},-y_{N}, 0\right),(0,0, z),(0,0,-z)\right),
\end{aligned}
$$


y el vector de velocidad del sistema está dado por

$$
\begin{aligned}
\mathbf{v} & =\dot{\mathbf{r}}=\left(\mathbf{v}_{1}, \ldots, \mathbf{v}_{N}, \mathbf{v}_{N+1}, \mathbf{v}_{N+2}\right) \\
& =\left(\left(\rho \dot{x}_{1}, \rho \dot{y}_{1}, 0\right), \ldots,\left(\rho \dot{x}_{N}, \rho \dot{y}_{N}, 0\right),(0,0, \dot{z}),(0,0,-\dot{z})\right)
\end{aligned}
$$

Una base natural para el espacio tangente del vector de configuración, r, está dada por los vectores $\left\{\underline{\alpha}_{1}, \underline{\beta}_{1}, \ldots, \underline{\alpha}_{N}, \underline{\beta}_{N}, \underline{\alpha}_{N+1}\right\}$, donde

$$
\begin{aligned}
\underline{\alpha}_{j} & =\left(\overline{0}, \ldots,(1,0,0), \ldots, \overline{0}_{N}, \overline{0}, \overline{0}\right), \\
\underline{\beta}_{j} & =\left(\overline{0}, \ldots,(0,1,0), \ldots, \overline{0}_{N}, \overline{0}, \overline{0}\right), \\
\underline{\alpha}_{N+1} & =\left(\overline{0}, \ldots, \overline{0}_{N},(0,0,1),(0,0,-1)\right) .
\end{aligned}
$$

Entonces podemos escribir el vector de velocidad del sistema como una combinación lineal de ellos de la siguiente forma

$$
\mathbf{v}=\rho \sum_{j=1}^{N}\left(\dot{x}_{j} \underline{\alpha}_{j}+\dot{y}_{j} \underline{\beta}_{j}\right)+\dot{z} \underline{\alpha}_{N+1},
$$

es decir, cualquier coordenada en el espacio tangente se escribe en términos de la base anterior.

Para encontrar la primer componente de la descomposición de la velocidad, $W_{1}$, primero tenemos que calcular el momento angular,

$$
\mathbf{c}=\left(c_{1}, c_{2}, c_{3}\right)=\left(\left\langle\mathbf{E}_{1} \times \mathbf{r}, \mathbf{v}\right\rangle,\left\langle\mathbf{E}_{2} \times \mathbf{r}, \mathbf{v}\right\rangle,\left\langle\mathbf{E}_{3} \times \mathbf{r}, \mathbf{v}\right\rangle\right)
$$

Realizando algunos cálculos, se obtiene que las tres componentes del momento angular son:

$$
\begin{aligned}
& c_{1}=\left\langle\left(\left(0,0, \rho y_{1}\right), \ldots,\left(0,0, \rho y_{N}\right), \overline{0}, \overline{0}\right)\right.\left.\left(\left(\rho \dot{x}_{1}, \rho \dot{y}_{1}, 0\right), \ldots,\left(\rho \dot{x}_{N}, \rho \dot{y}_{N}, 0\right),(0,0, \dot{z}),(0,0,-\dot{z})\right)\right\rangle=0, \\
& c_{2}=\left\langle\left(\left(0,0,-\rho x_{1}\right), \ldots,\left(0,0,-\rho x_{N}\right), \overline{0}, \overline{0}\right),\right. \\
&\left.\quad\left(\left(\rho \dot{x}_{1}, \rho \dot{y}_{1}, 0\right), \ldots,\left(\rho \dot{x}_{N}, \rho \dot{y}_{N}, 0\right),(0,0, \dot{z}),(0,0,-\dot{z})\right)\right\rangle=0, \\
& c_{3}=\left\langle\left(\left(-\rho y_{1}, \rho x_{1}, 0\right), \ldots,\left(-\rho y_{N}, \rho x_{N}, 0\right), \overline{0}, \overline{0}\right),\right. \\
&\left.\quad\left(\left(\rho \dot{x}_{1}, \rho \dot{y}_{1}, 0\right), \ldots,\left(\rho \dot{x}_{N}, \rho \dot{y}_{N}, 0\right),(0,0, \dot{z}),(0,0,-\dot{z})\right)\right\rangle \\
&=N m_{1} \rho^{2} \sum_{j=1}^{N}\left(x_{j} \dot{y}_{j}-y_{j} \dot{x}_{j}\right),
\end{aligned}
$$


por lo cual, el momento angular para el problema romboidal generalizado en rotación es

$$
\mathbf{c}=\left(0,0, N m_{1} \rho^{2} \sum_{j=1}^{N}\left(x_{j} \dot{y}_{j}-y_{j} \dot{x}_{j}\right)\right)
$$

el cual únicamente tiene componente distinta de cero en dirección del eje $z$. Entonces, resulta que al proyectar $\mathbf{v}$ en cada uno de los vectores $\mathbf{E}_{i} \times \mathbf{r}$ con $i=1,2,3$, el vector de velocidad rotacional esta dado por

$$
W_{1}=\sum_{i=1}^{3} \frac{\left\langle\mathbf{E}_{i} \times \mathbf{r}, \mathbf{v}\right\rangle}{\left|\mathbf{E}_{i} \times \mathbf{r}\right|} \frac{\mathbf{E}_{i} \times \mathbf{r}}{\left|\mathbf{E}_{i} \times \mathbf{r}\right|}=\sum_{i=1}^{3} \frac{c_{i}}{\left|\mathbf{E}_{i} \times \mathbf{r}\right|} \frac{\mathbf{E}_{i} \times \mathbf{r}}{\left|\mathbf{E}_{i} \times \mathbf{r}\right|}=\sum_{i=1}^{3} \frac{\mathbf{e}_{i} \cdot \mathbf{c}}{\left|\mathbf{E}_{i} \times \mathbf{r}\right|} \frac{\mathbf{E}_{i} \times \mathbf{r}}{\left|\mathbf{E}_{i} \times \mathbf{r}\right|}
$$

pero como las primeras dos componentes del momento angular son cero, $c_{1}=c_{2}=0$, obtenemos que $W_{1}$ se escribe de la siguiente forma

$$
W_{1}=\frac{\mathbf{c}}{\left|\mathbf{E}_{3} \times \mathbf{r}\right|} \frac{\mathbf{E}_{3} \times \mathbf{r}}{\left|\mathbf{E}_{3} \times \mathbf{r}\right|},
$$

donde $\mathbf{c}$ es la componente del momento angular en dirección del eje $z$, la cual en efecto, es el momento angular, implicando que en este sistema el vector de velocidad rotacional $W_{1}$, viva en el subespacio $S_{\text {rot }}=\operatorname{span}\left\{\mathbf{E}_{3} \times \mathbf{r}\right\}$, por lo cual su dimensión es uno, $\operatorname{dim}\left(S_{\text {rot }}\right)=1$.

Además se tiene que

$$
\begin{aligned}
\mathbf{E}_{3} \times \mathbf{r} & =\left(\left(-\rho y_{1}, \rho x_{1}, 0\right), \ldots,\left(-\rho y_{N}, \rho x_{N}, 0\right), \overline{0}, \overline{0}\right)=\rho \sum_{j=1}^{N}\left(x_{j} \underline{\beta}_{j}-y_{j} \underline{\alpha}_{j}\right), \\
\left|\mathbf{E}_{3} \times \mathbf{r}\right|^{2} & =\left\langle\mathbf{E}_{3} \times \mathbf{r}, \mathbf{E}_{3} \times \mathbf{r}\right\rangle=N m_{1} \rho^{2},
\end{aligned}
$$

por lo tanto, sustituyendo lo anterior en la ecuación (4.2) se tiene que la componente de la velocidad dedicada a rotar las $N$-partículas como un cuerpo rígido del problema romboidal generalizado en rotación es

$$
W_{1}(\mathbf{r}, \mathbf{c})=\lambda_{1} \frac{\rho \sum_{j=1}^{N}\left(x_{j} \underline{\beta}_{j}-y_{j} \underline{\alpha}_{j}\right)}{\left(N m_{1} \rho^{2}\right)^{1 / 2}},
$$

donde

$$
\lambda_{1}=\frac{\mathbf{c}}{\left|E_{3} \times \mathbf{r}\right|}=\frac{\mathbf{c}}{\left(N m_{1} \rho^{2}\right)^{1 / 2}} .
$$

Hemos obtenido la primer componente de la descomposición de la velocidad de Saari para el problema romboidal generalizado en rotación, $W_{1}$, la cual se encuentra en términos del vector $\mathbf{E}_{3} \times \mathbf{r}$, puesto que al hacer la proyección del vector de velocidad del sistema, $\mathbf{v}$, a cada uno de los vectores $\mathbf{E}_{i} \times \mathbf{r}$ con $i=1,2,3$, resulta que la única componente de la proyección que es distinta de cero, es la correspondiente a la tercer componente del momento angular, debido a que las partículas sólo están rotando alrededor del eje $z$. 
Por otro lado, la velocidad escalar, $W_{2}$, dada por la ecuación (2.10) es

$$
W_{2}=\lambda_{2} \frac{\mathbf{r}}{|\mathbf{r}|}
$$

donde $\lambda_{2}=\frac{\dot{I}}{2 \sqrt{I}}$, y para la cual se tiene que

$$
\begin{aligned}
I & =\langle\mathbf{r}, \mathbf{r}\rangle=|\mathbf{r}|^{2}=m_{1} \rho^{2}\left(x_{1}^{2}+y_{1}^{2}+\ldots+x_{N}^{2}+y_{N}^{2}\right)+2 m_{2} z^{2} \\
& =m_{1} \rho^{2}\left(\left|\left(x_{1}, y_{1}\right)\right|^{2}+\ldots+\left|\left(x_{N}, y_{N}\right)\right|^{2}\right)+2 m_{2} z^{2} \\
& =N m_{1} \rho^{2}+2 m_{2} z^{2}
\end{aligned}
$$

y además que

$$
\dot{I}=4 m_{2} z \dot{z}
$$

implicando que

$$
\lambda_{2}=\frac{2 m_{2} z \dot{z}}{\left(N m_{1} \rho^{2}+2 m_{2} z^{2}\right)^{1 / 2}} .
$$

Por lo tanto, la componente de la velocidad que representa el cambio en el tamaño de la configuración del problema romboidal generalizado en rotación es

$$
W_{2}=\lambda_{2} \frac{\left(\rho\left(x_{1}, y_{1}, 0\right), \rho\left(-x_{1},-y_{1}, 0\right), \ldots, \rho\left(-x_{N},-y_{N}, 0\right),(0,0, z),(0,0,-z)\right)}{|\mathbf{r}|}
$$

El subespacio donde vive $W_{2}$ es $S_{\text {scal }}$, el cual siempre tiene dimensión uno, y se encuentra en el complemento ortogonal de $S_{\text {rot }}$, es decir,

$$
S_{\text {scal }}(\mathbf{r})=\left\{t \frac{\mathbf{r}}{|\mathbf{r}|}: t \in \mathbb{R}\right\} \subseteq\left(S_{\text {rot }}\right)^{\perp}
$$

puesto que todo elemento de $S_{\text {scal }}$ es ortogonal al conjunto que genera a $S_{\text {rot }}$, esto es,

$$
\left\langle\mathbf{E}_{3} \times \mathbf{r}, t \frac{\mathbf{r}}{|\mathbf{r}|}\right\rangle=\frac{t}{|\mathbf{r}|}\left\langle\mathbf{E}_{3} \times \mathbf{r}, \mathbf{r}\right\rangle=\frac{t}{|\mathbf{r}|}\left\langle\mathbf{E}_{3}, \mathbf{r} \times \mathbf{r}\right\rangle=0 .
$$

El vector $W_{2}(\mathbf{r}, I)$ vive en el espacio tangente del espacio de configuración, $T_{\mathbf{r}}(C S)$, abusando de la notación podemos escribir a $\mathbf{r}$ como una combinación lineal de la base para el espacio tangente del vector de configuración $\mathbf{r}$, es decir,

$$
\mathbf{r}=\rho \sum_{j=1}^{N}\left(x_{j} \underline{\alpha}_{j}+y_{j} \underline{\beta}_{j}\right)+z \underline{\alpha}_{N+1} .
$$

Sabemos que podemos obtener el vector $W_{3}$, encargado de determinar cómo cambia la forma de la configuración del problema romboidal generalizado en rotación de la siguiente expresión

$$
W_{3}=\mathbf{v}-\left(W_{1}+W_{2}\right)
$$


sin embargo, obtendremos éste como una combinación lineal de la base dada por los vectores de las ecuaciones (4.1), y aprovecharemos la ortogonalidad entre los vectores de la descomposición de la velocidad, es decir,

$$
W_{3}=\sum_{j=1}^{N}\left(a_{j} \underline{\alpha}_{j}+b_{j} \underline{\beta}_{j}\right)+d \underline{\alpha}_{N+1},
$$

y usando la ortogonalidad tenemos que $\left\langle W_{1}, W_{3}\right\rangle=0$, obteniendo

$$
\begin{aligned}
\left\langle W_{1}, W_{3}\right\rangle= & \frac{\lambda_{1} \rho}{\left|\mathbf{E}_{3} \times \mathbf{r}\right|}\left\langle\sum_{j=1}^{N}\left(\dot{x}_{j} \underline{\beta}_{j}-\dot{y}_{j} \underline{\alpha}_{j}\right), \sum_{j=1}^{N}\left(a_{j} \underline{\alpha}_{j}+b_{j} \underline{\beta}_{j}\right)+d \underline{\alpha}_{N+1}\right\rangle \\
= & \frac{\lambda_{1} \rho m_{1}}{\left|\mathbf{E}_{3} \times \mathbf{r}\right|}\left\langle\left(-\dot{y}_{1}, \dot{x}_{1}, 0, \ldots,-\dot{y}_{N}, \dot{x}_{N}, 0, \overline{0}, \overline{0}\right),\right. \\
& \left.\left(a_{1}, b_{1}, 0, \ldots, a_{N}, b_{N}, 0,0,0, d, 0,0,-d\right)\right\rangle \\
= & \frac{\lambda_{1} \rho m_{1}}{\left|\mathbf{E}_{3} \times \mathbf{r}\right|}\left(b_{1} \dot{x}_{1}-a_{1} \dot{y}_{1}+\ldots+b_{N} \dot{x}_{N}-a_{N} \dot{y}_{N}\right)=0,
\end{aligned}
$$

luego $\left|\underline{\alpha}_{j}\right|^{2}=m_{1}=\left|\underline{\beta}_{j}\right|^{2}$, de aquí podemos escribir la ecuación anterior como

$$
b_{1} \dot{x}_{1}\left|\underline{\beta}_{j}\right|^{2}-a_{1} \dot{y}_{1}\left|\underline{\alpha}_{j}\right|^{2}+\ldots+b_{N} \dot{x}_{N}\left|\underline{\beta}_{j}\right|^{2}-a_{N} \dot{y}_{N}\left|\underline{\alpha}_{j}\right|^{2}=0
$$

y como $\left|\underline{\alpha}_{j}\right|=\left|\underline{\beta}_{j}\right|$, se obtiene

$$
b_{j} \dot{x}_{j}-a_{j} \dot{y}_{j}=0
$$

por lo tanto, hemos obtenido que

$$
a_{j}=\dot{x}_{j} \quad \mathrm{y} \quad b_{j}=\dot{y}_{j}
$$

De la misma forma, utilizando que $\left\langle W_{2}, W_{3}\right\rangle=0$, llegamos a la siguiente igualdad

$$
\rho m_{1} \sum_{j=1}^{N}\left(x_{j} \dot{x}_{j}+y_{j} \dot{y}_{j}\right)+2 m_{2} z d=0,
$$

por lo tanto, se obtiene que

$$
d=-\frac{\rho m_{1} \sum_{j=1}^{N}\left(x_{j} \dot{x}_{j}+y_{j} \dot{y}_{j}\right)}{2 m_{2} z} .
$$

Sustituyendo los resultados de las ecuaciones (4.5) y (4.6), en la ecuación (4.4) tenemos

$$
W_{3}=\sum_{j=1}^{N}\left(\dot{x}_{j} \underline{\alpha}_{j}+\dot{y}_{j} \underline{\beta}_{j}\right)-\frac{\rho m_{1} \sum_{j=1}^{N}\left(x_{j} \dot{x}_{j}+y_{j} \dot{y}_{j}\right)}{2 m_{2} z} \underline{\alpha}_{N+1},
$$


luego, con el propósito de eliminar la singularidad, multiplicamos la expresión anterior por $2 m_{2} z$, y renombrando $W_{3}$ como

$$
\bar{W}_{3}=2 m_{2} z W_{3}=2 m_{2} z \sum_{j=1}^{N}\left(\dot{x}_{j} \underline{\alpha}_{j}+\dot{y}_{j} \underline{\beta}_{j}\right)-\rho m_{1} \sum_{j=1}^{N}\left(x_{j} \dot{x}_{j}+y_{j} \dot{y}_{j}\right) \underline{\alpha}_{N+1},
$$

el cual en términos de vectores unitarios puede ser escrito de la siguiente forma

$$
\bar{W}_{3}=\lambda_{3} \frac{\bar{W}_{3}}{\left|\bar{W}_{3}\right|}
$$

donde $\lambda_{3}$ se obtiene de la integral de energía dada por la ecuación (2.5), es decir, hemos obtenido la siguiente descomposición ortogonal del vector de velocidad del problema romboidal generalizado en rotación

$$
\mathbf{v}=\lambda_{1} \frac{E_{3} \times \mathbf{r}}{\left|E_{3} \times \mathbf{r}\right|}+\lambda_{2} \frac{\mathbf{r}}{|\mathbf{r}|}+\lambda_{3} \frac{\bar{W}_{3}}{\left|\bar{W}_{3}\right|}
$$

entonces

$$
\langle\mathbf{v}, \mathbf{v}\rangle=\lambda_{1}^{2}+\lambda_{2}^{2}+\lambda_{3}^{2}=2(U+h)
$$

por lo tanto,

$$
\lambda_{3}^{2}=2(U+h)-\left(\lambda_{1}^{2}+\lambda_{2}^{2}\right),
$$

donde la función potencial, $U$, para el problema romboidal generalizado tiene la siguiente expresión

$$
U=\frac{m_{2}^{2}}{2 z}+\frac{2 N m_{1} m_{2}}{\left(\rho^{2}+z^{2}\right)^{1 / 2}}+\sum_{k<j \leq N} \frac{m_{1}^{2}}{\left|\mathbf{r}_{j}-\mathbf{r}_{k}\right|}
$$

Hemos obtenido una representación para los tres vectores de la descomposición ortogonal de la velocidad del problema romboidal generalizado en rotación, algunos de los resultados obtenidos en esta sección, serán utilizados más adelante para estudiar la dinámica de este problema.

\subsection{Componentes de la velocidad: Problema isósceles en rotación}

Consideremos nuevamente el problema isósceles en rotación, es decir, tenemos dos partículas de masa $m_{1}$ ubicadas sobre el plano $x$ - $y$ las cuales se encuentran simétricamente localizadas respecto al origen y están rotando al rededor del eje $z$ con una velocidad angular constante. Supongamos que la tercer partícula tiene masa $m_{2}$ y se encuentra localizada sobre el eje $z$ a una distancia con respecto al origen de $\sqrt{\frac{M}{2 m_{1}}}$ donde $M$ es la masa total del sistema $M=2 m_{1}+m_{2}$, véase figura 3.2 . 
En este caso, los vectores de posición están dados por

$$
\mathbf{r}_{1}=(x, y, 0), \quad \mathbf{r}_{2}=(-x,-y, 0), \quad \mathbf{r}_{3}=\left(0,0, z \sqrt{\frac{M}{2 m_{1}}}\right)
$$

El vector de posición del sistema está dado por $\mathbf{r}=\left(\mathbf{r}_{1}, \mathbf{r}_{2}, \mathbf{r}_{3}\right)$, y el vector velocidad del sistema lo escribimos como

$$
\mathbf{v}=\dot{\mathbf{r}}=\left((\dot{x}, \dot{y}, 0),(-\dot{x},-\dot{y}, 0,),\left(0,0, \dot{z} \sqrt{\frac{M}{2 m_{1}}}\right)\right),
$$

sea $\left\{\underline{\alpha}_{1}, \underline{\alpha}_{2}, \underline{\alpha}_{3}\right\}$ una base natural para el espacio tangente del vector de configuración, $\mathbf{r}$, donde

$$
\begin{aligned}
& \underline{\alpha}_{1}=((1,0,0),(-1,0,0), \overline{0}), \\
& \underline{\alpha}_{2}=((0,1,0),(0,-1,0), \overline{0}), \\
& \underline{\alpha}_{3}=\left(\overline{0}, \overline{0},\left(0,0, \sqrt{\frac{M}{2 m_{1}}}\right)\right),
\end{aligned}
$$

entonces podemos escribir el vector de velocidades como una combinación lineal de ellos, esto es,

$$
\mathbf{v}=\dot{x} \underline{\alpha}_{1}+\dot{y} \underline{\alpha}_{2}+\dot{z} \underline{\alpha}_{3} .
$$

Como ya hemos dicho, podría pensarse que el problema isósceles en rotación es similar al romboidal generalizado en rotación, y que no existe razón para obtener las componentes de la descomposición de la velocidad, sin embargo, recordemos que el centro de masas del problema isósceles en rotación se encuentra siempre sobre el eje $z$, es decir,

$$
\mathbf{c}_{m}=m_{2}\left(0,0, z \sqrt{\frac{M}{2 m_{1}}}\right),
$$

y como es usual, lo fijamos en el origen.

Para iniciar con la descomposición ortogonal del vector de velocidad, $\mathbf{v}$, calculemos las tres componentes del momento angular

$$
\begin{aligned}
c_{1} & =\left\langle\mathbf{E}_{1} \times \mathbf{r}, \mathbf{v}\right\rangle=0, \\
c_{2} & =\left\langle\mathbf{E}_{2} \times \mathbf{r}, \mathbf{v}\right\rangle=0, \\
c_{3} & =\left\langle((-y, x, 0),(y,-x, 0), \overline{0}),\left((\dot{x}, \dot{y}, 0),(-\dot{x},-\dot{y}, 0),\left(0,0, \dot{z} \sqrt{\frac{M}{2 m_{1}}}\right)\right)\right\rangle \\
& =2 m_{1}(\dot{y} x-\dot{x} y) .
\end{aligned}
$$

Como era de esperar sólo existe componente en dirección del eje $z$, ya que las partículas sobre el plano $x-y$ están rotando al rededor del eje $z$, por lo tanto el momento angular es

$$
\mathbf{c}=\left(c_{1}, c_{2}, c_{3}\right)=\left(0,0,2 m_{1}(\dot{y} x-\dot{x} y)\right),
$$


así el vector de velocidad rotacional queda expresado de la siguiente forma

$$
W_{1}=\frac{\mathbf{c}}{\left|E_{3} \times \mathbf{r}\right|} \frac{E_{3} \times \mathbf{r}}{\left|E_{3} \times \mathbf{r}\right|}
$$

donde c representa la componente del momento angular en dirección del eje z. Luego,

$$
\left|\mathbf{E}_{3} \times \mathbf{r}\right|^{2}=2 m_{1}\left(x^{2}+y^{2}\right)
$$

de esta expresión se obtiene que la componente de la velocidad dedicada a las rotaciones del problema isósceles es

$$
W_{1}=\lambda_{1} \frac{(-y, x, 0, y,-x, 0, \overline{0})}{\left|E_{3} \times \mathbf{r}\right|},
$$

donde

$$
\lambda_{1}=\frac{\mathbf{c}}{\left|E_{3} \times \mathbf{r}\right|}=\frac{\mathbf{c}}{\left(2 m_{1}\left(x^{2}+y^{2}\right)\right)^{1 / 2}} .
$$

En este caso el vector $W_{1}$, vive en el subespacio $S_{\text {rot }}=\operatorname{span}\left\{\mathbf{E}_{3} \times \mathbf{r}\right\}$, por lo cual su dimensión es uno, $\operatorname{dim}\left(S_{\text {rot }}\right)=1$.

Ya hemos visto que la componente de la velocidad que representa el cambio en la escala del sistema, está dada por la relación

$$
W_{2}=\lambda_{2} \frac{\mathbf{r}}{|\mathbf{r}|}
$$

donde $\lambda_{2}=\frac{\dot{I}}{2 \sqrt{I}}$, y en este caso tenemos que el momento de inercia y su razón de cambio con respecto al tiempo son

$$
\begin{aligned}
& I=|\mathbf{r}|^{2}=2 m_{1}\left(x^{2}+y^{2}\right)+m_{2} \frac{M}{2 m_{1}} z^{2}, \\
& \dot{I}=2\left[2 m_{1}(x \dot{x}+y \dot{y})+m_{2} \frac{M}{2 m_{1}} z \dot{z}\right],
\end{aligned}
$$

respectivamente. Por lo tanto, la componente de la velocidad dedicada a cambiar el tamaño en la escala de la configuración del problema isósceles en rotación está dada por

$$
W_{2}=\frac{\lambda_{2}}{|\mathbf{r}|}\left(x, y, 0,-x,-y, 0,0,0, z \sqrt{\frac{M}{2 m_{1}}}\right),
$$

donde $\lambda_{2}=\frac{2 m_{1}(x \dot{x}+y \dot{y})+m_{2} \frac{M}{2 m_{1}} z \dot{z}}{\left(2 m_{1}\left(x^{2}+y^{2}\right)+m_{2} \frac{M}{2 m_{1}} z^{2}\right)^{1 / 2}}$.

El vector $W_{2}$ vive en el subespacio $S_{\text {scal }}$, el cual siempre tiene dimensión uno, y se encuentra en el complemento ortogonal de $S_{\text {rot }}$, esto es,

$$
S_{\text {scal }}(\mathbf{r})=\left\{t \frac{\mathbf{r}}{|\mathbf{r}|}: t \in \mathbb{R}\right\} \subseteq\left(S_{\text {rot }}\right)^{\perp} .
$$

Otra forma de decirlo es la siguiente, el vector $W_{2}(\mathbf{r}, I)$ vive en el espacio tangente del espacio de configuración, $T_{\mathbf{r}}(C S)$, abusando de la notación podemos escribir

$$
\mathbf{r}=x \underline{\alpha}_{1}+y \underline{\alpha}_{2}+z \underline{\alpha}_{3} .
$$


En lo que sigue obtendremos el vector $W_{3}$ dedicado al cambio en la configuración. Sabemos que es posible obtener el vector $W_{3}$ de la siguiente expresión

$$
W_{3}=\mathbf{v}-\left(W_{1}+W_{2}\right),
$$

sin embargo, lo obtendremos como una combinación lineal de la base dada por los vectores de la ecuación (4.8), entonces primero escribimos éste como una combinación lineal de dicha base, es decir,

$$
W_{3}=a \underline{\alpha}_{1}+b \underline{\alpha}_{2}+d \underline{\alpha}_{3},
$$

luego, como $\left\langle W_{1}, W_{3}\right\rangle=0$, se tiene

$$
\left\langle\lambda_{1}\left(\dot{x} \underline{\alpha}_{2}-\dot{y} \underline{\alpha}_{1}\right), a \underline{\alpha}_{1}+b \underline{\alpha}_{2}+d \underline{\alpha}_{3}\right\rangle=2 m_{1} \lambda_{1}(b \dot{x}-a \dot{y})=0,
$$

además $\left|\underline{\alpha}_{1}\right|^{2}=2 m_{1}=\left|\underline{\alpha}_{2}\right|^{2}$, de aquí

$$
2 m_{1} b \dot{x}-2 m_{1} a \dot{y}=b \dot{x}\left|\lambda_{2}\right|^{2}-a \dot{y}\left|\lambda_{1}\right|^{2}=0,
$$

por lo tanto,

$$
-a \dot{y}+b \dot{x}=0
$$

obteniendo

$$
a=\dot{x} \quad \mathrm{y} \quad b=\dot{y} .
$$

Después, como también $\left\langle W_{2}, W_{3}\right\rangle=0$ se tiene

$$
\left\langle\frac{\lambda_{2}}{|\mathbf{r}|} \mathbf{r}, a \underline{\alpha}_{1}+b \underline{\alpha}_{2}+d \underline{\alpha}_{3}\right\rangle=\frac{\lambda_{2}}{|\mathbf{r}|}\left(2 m_{1}(x \dot{x}+y \dot{y})+d m_{2} \frac{M}{2 m_{1}} z\right)=0,
$$

y de allí despejando $d$ se obtiene

$$
d=\frac{-4 m_{1}^{2}(x \dot{x}+y \dot{y})}{M m_{2} z}
$$

Sustituyendo los resultados de las ecuaciones (4.10) y (4.11), en la ecuación (4.9) obtenemos

$$
W_{3}=\dot{x} \underline{\alpha}_{1}+\dot{y} \underline{\alpha}_{2}-\frac{4 m_{1}^{2}(x \dot{x}+y \dot{y})}{M m_{2} z} \underline{\alpha}_{3},
$$

para eliminar la singularidad, multiplicamos la expresión anterior por $M m_{2} z$, y renombrando $W_{3}$ como

$$
\bar{W}_{3}=M m_{2} z W_{3}=M m_{2} \dot{x} z \underline{\alpha}_{1}+M m_{2} \dot{y} z \underline{\alpha}_{2}-4 m_{1}^{2}(x \dot{x}+y \dot{y}) \underline{\alpha}_{3},
$$

el cual en términos de vectores unitarios puede ser escrito de la siguiente forma

$$
\bar{W}_{3}=\lambda_{3} \frac{\bar{W}_{3}}{\left|\bar{W}_{3}\right|}
$$

donde $\lambda_{3}$ la obtenemos nuevamente de la integral de energía

$$
\lambda_{3}^{2}=2(U+h)-\left(\lambda_{1}^{2}+\lambda_{2}^{2}\right) .
$$


Por otro lado, después de realizar algunos cálculos, obtenemos que la función potencial del problema isósceles en rotación es

$$
U=\frac{m_{1}^{2}}{2\left(x^{2}+y^{2}\right)^{1 / 2}}+\frac{2 m_{1} m_{2}}{\left(x^{2}+y^{2}+\frac{M}{2 m_{1}} z^{2}\right)^{1 / 2}} .
$$

En conclusión, encontramos una representación para las tres componentes de la descomposición ortogonal de la velocidad, $W_{1}, W_{2}$ y $W_{3}$. Más adelante utilizaremos algunos de los resultados obtenidos, con el propósito de estudiar la dinámica del problema isósceles en rotación.

\subsection{Componentes de la velocidad: Problema colineal de 3-cuerpos en el plano}

Por último, estudiamos la descomposición de la velocidad de Saari del problema colineal de 3 -cuerpos en el plano. Recordemos, tenemos tres partículas con posiciones $\mathbf{r}_{1}, \mathbf{r}_{2}, \mathbf{r}_{3}$ con masas distintas $m_{1}, m_{2}$ y $m_{3}$ respectivamente, las cuales se encuentran colocadas de tal forma que siempre están sobre una linea recta en el plano $x-y$, la interpretación geométrica se observa en la figura 4.1 (a).

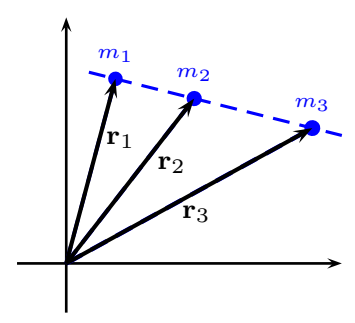

(a) Tres partículas colineales $\mathbf{r}_{1}, \mathbf{r}_{2}$ y $\mathbf{r}_{3}$ en el plano $x-y$.

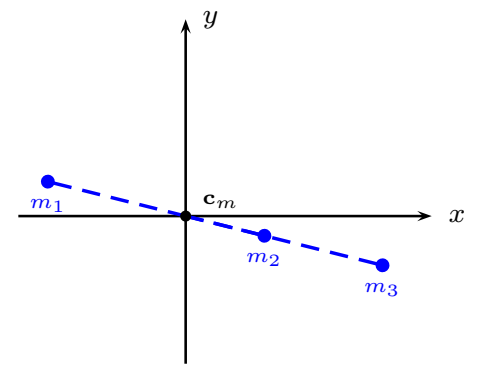

(b) Tres partículas con masas $m_{1}$, $m_{2}$ y $m_{3}$, sobre una linea recta que pasa por el origen.

Figura 4.1: Problema Colineal de 3-cuerpos en el plano.

Ahora, definiendo los vectores de posición de la forma

$$
\begin{aligned}
& \mathbf{q}_{1}=\mathbf{r}_{2}-\mathbf{r}_{1}, \\
& \mathbf{q}_{2}=\mathbf{r}_{3}-\mathbf{r}_{2}, \\
& \mathbf{q}_{3}=\mathbf{r}_{3}-\mathbf{r}_{1}=\left(\mathbf{r}_{3}-\mathbf{r}_{2}\right)+\left(\mathbf{r}_{2}-\mathbf{r}_{1}\right)=\mathbf{q}_{1}+\mathbf{q}_{2},
\end{aligned}
$$

obtenemos que la línea recta que contiene a las partículas en las posiciones $\mathbf{q}_{1}, \mathbf{q}_{2}$ y $\mathbf{q}_{3}$ pasa por el origen, y en el cual se encuentra fijo el centro de masas, la interpretación geométrica se observa en la figura 4.1 (b). 
Lo anterior, nos permite escribir

$$
\begin{aligned}
& \mathbf{q}_{1}=\mathbf{r}_{2}-\mathbf{r}_{1}=(x, y, 0) \\
& \mathbf{q}_{2}=\alpha\left(\mathbf{r}_{2}-\mathbf{r}_{1}\right)=\alpha(x, y, 0) \\
& \mathbf{q}_{3}=(\alpha+1)\left(\mathbf{r}_{2}-\mathbf{r}_{1}\right)=(\alpha+1)(x, y, 0)
\end{aligned}
$$

donde $\alpha \in \mathbb{R}$. Consideremos un primer caso, supongamos que las tres partículas no rotan de ninguna forma posible, es decir, éstas sólo permanecen sobre la misma línea recta para todo tiempo $t$; por lo cual el momento angular es cero, $\mathbf{c}=(0,0,0)$, entonces la primer componente de la velocidad dedicada a las rotaciones del sistema vistas como un cuerpo rígido es cero, $W_{1}=0$, lo cual nos lleva a encontrar únicamente los vectores de velocidad escalar y velocidad configuracional, $W_{2}$ y $W_{3}$, respectivamente.

El vector que describe la configuración formada por las 3-partículas, está dado por

$$
\mathbf{q}=(x, y, 0, \alpha x, \alpha y, 0,(\alpha+1) x,(\alpha+1) y, 0) .
$$

La velocidad del sistema está dada por

$$
\mathbf{v}=\dot{\mathbf{q}}=\left(\dot{\mathbf{q}}_{1}, \dot{\mathbf{q}}_{2}, \dot{\mathbf{q}}_{3}\right) .
$$

Una base natural para el espacio tangente de q, está dada por

$$
\left\{\underline{\beta}_{1}=(1,0,0, \alpha, 0,0, \alpha+1,0,0), \underline{\beta}_{2}=(0,1,0,0, \alpha, 0,0, \alpha+1,0)\right\},
$$

por lo cual podemos escribir el vector de velocidad del sistema, $\mathbf{v}$, como una combinación lineal de ésta,

$$
\mathbf{v}=\dot{x} \underline{\beta}_{1}+\dot{y} \underline{\beta}_{2} .
$$

Como ya se mencionó, en este primer caso estamos considerando que el momento angular es cero, entonces la componente de la velocidad dedicada a las rotaciones del sistema es cero. Respecto a la velocidad escalar, $W_{2}$, la cual representa el cambio en el tamaño del sistema, es decir, la forma de la configuración nunca cambia solamente su escala, está dada por

$$
W_{2}(\mathbf{q}, I)=\frac{\dot{I}}{2 \sqrt{I}} \frac{\mathbf{q}}{|\mathbf{q}|}=\lambda_{2} \frac{\mathbf{q}}{|\mathbf{q}|},
$$

donde realizando algunos cálculos, se llega

$$
\begin{aligned}
& I=\langle\mathbf{q}, \mathbf{q}\rangle=|\mathbf{q}|^{2}=\left[m_{1}+m_{2} \alpha^{2}+m_{3}(\alpha+1)^{2}\right]\left(x^{2}+y^{2}\right), \\
& \dot{I}=2\left[m_{1}+m_{2} \alpha^{2}+m_{3}(\alpha+1)^{2}\right](x \dot{x}+y \dot{y}),
\end{aligned}
$$

implicando que

$$
\lambda_{2}=\frac{\left[m_{1}+m_{2} \alpha^{2}+m_{3}(\alpha+1)^{2}\right]^{1 / 2}(x \dot{x}+y \dot{y})}{\left(x^{2}+y^{2}\right)^{1 / 2}},
$$

por lo tanto, el vector de velocidad escalar para el problema colineal de tres cuerpos en el plano es

$$
W_{2}(\mathbf{q}, I)=\frac{\lambda_{2}}{|\mathbf{q}|}(x, y, 0, \alpha x, \alpha y, 0,(\alpha+1) x,(\alpha+1) y, 0) .
$$


Recordemos que el vector $W_{2}(\mathbf{q}, I)$ vive en el subespacio $S_{\text {rot }}$, el cual siempre tiene dimensión uno, también se puede decir que $W_{2}$, vive en el espacio tangente del espacio de configuración, $T_{\mathbf{r}}(C S)$, abusando de la notación podemos escribir

$$
\mathbf{q}=x \underline{\beta}_{1}+y \underline{\beta}_{2} .
$$

Con el propósito de obtener $W_{3}$, nuevamente escribimos éste como una combinación lineal de la base anterior,

$$
W_{3}=a \underline{\beta}_{1}+b \underline{\beta}_{2}
$$

luego usando el hecho de que $\left\langle W_{2}, W_{3}\right\rangle=0$, puesto que cada uno se encuentra en los subespacios ortogonales $S_{\text {scal }}$ y $S_{\text {conf }}$ respectivamente, tenemos

$$
\begin{aligned}
&\left\langle\lambda_{2} \frac{\mathbf{q}}{|\mathbf{q}|}, a \underline{\beta}_{1}+b \underline{\beta}_{2}\right\rangle=\frac{\lambda_{2}}{|\mathbf{q}|}\left\langle\mathbf{q}, a \underline{\beta}_{1}+b \underline{\beta}_{2}\right\rangle \\
&=\langle(\dot{x}, \dot{y}, 0, \alpha \dot{x}, \alpha \dot{y}, 0,(\alpha+1) \dot{x},(\alpha+1) \dot{y}, 0) \\
&\quad(a, b, 0, a \alpha, b \alpha, 0, a(\alpha+1), b(\alpha+1), 0)\rangle=0
\end{aligned}
$$

obteniendo

$$
\left[m_{1}+m_{2} \alpha^{2}+m_{3}(\alpha+1)^{2}\right](a \dot{x}+b \dot{y})=0,
$$

resolviendo la ecuación anterior se llega: $a=\dot{y}$ y $b=-\dot{x}$, ó, $a=-\dot{y}$ y $b=\dot{x}$. Por lo tanto, la velocidad configuracional es

$$
W_{3}=\dot{y} \underline{\beta}_{1}-\dot{x} \underline{\beta}_{2},
$$

de aquí renombrando $W_{3}$ para escribir éste en términos de vectores unitarios, se tiene

$$
\bar{W}_{3}=\lambda_{3} \frac{W_{3}}{\left|W_{3}\right|}
$$

donde $\lambda_{3}$ se obtiene de la integral de energía dada por la ecuación (2.5), esto es,

$$
\mathbf{v}=\lambda_{2} \frac{\mathbf{r}}{|\mathbf{r}|}+\lambda_{3} \frac{W_{3}}{\left|W_{3}\right|}
$$

entonces

$$
\langle\mathbf{v}, \mathbf{v}\rangle=\lambda_{2}^{2}+\lambda_{3}^{2}=2(U+h)
$$

por lo tanto,

$$
\lambda_{3}^{2}=2(U+h)-\lambda_{2}^{2},
$$

donde la función potencial $U$, para el problema colineal de tres cuerpos en el plano, tiene la siguiente expresión

$$
U(x, y)=\frac{m_{1} m_{2}|\alpha|+m_{2} m_{3}|\alpha||1-\alpha|+m_{1} m_{3}|1-\alpha|}{|\alpha||1-\alpha|\left(x^{2}+y^{2}\right)^{1 / 2}} .
$$

Un segundo caso, es considerar que las partículas tienen movimiento de rotación, es decir, las tres partículas están rotando en el plano $x-y$ con la restricción de permanecer siempre sobre 
una linea recta, la cual estará cambiando conforme las partículas están rotando; entonces calculando las tres componentes del vector momento angular tenemos

$$
\begin{aligned}
c_{1} & =\left\langle\mathbf{E}_{1} \times \mathbf{q}, \mathbf{v}\right\rangle \\
& =\langle(0,0, y, 0,0, \alpha y, 0,0,(\alpha+1) y),(\dot{x}, \dot{y}, 0, \alpha \dot{x}, \alpha \dot{y}, 0,(\alpha+1) \dot{x},(\alpha+1) \dot{y}, 0)\rangle=0, \\
c_{2} & =\left\langle\mathbf{E}_{2} \times \mathbf{q}, \mathbf{v}\right\rangle=\langle(0,0,-x, 0,0,-\alpha x, 0,0,-(\alpha+1) x), \mathbf{v}\rangle=0, \\
c_{3} & =\left\langle\mathbf{E}_{3} \times \mathbf{q}, \mathbf{v}\right\rangle=\langle(-y, x, 0,-\alpha y, \alpha x, 0,-(\alpha+1) y,(\alpha+1) x, 0), \mathbf{v}\rangle \\
& =\left[m_{1}+m_{2} \alpha^{2}+m_{3}(\alpha+1)^{2}\right](x \dot{y}-y \dot{x}),
\end{aligned}
$$

por lo tanto, hemos obtenido la siguiente expresión para el momento angular

$$
\mathbf{c}=\left(0,0,\left[m_{1}+m_{2} \alpha^{2}+m_{3}(\alpha+1)^{2}\right](x \dot{y}-y \dot{x})\right) .
$$

Como ya vimos las partículas se encuentran rotando alrededor de un vector paralelo al eje $z$, y conforme a lo estudiado anteriormente el vector de velocidad rotacional es

$$
W_{1}(\mathbf{q}, \mathbf{c})=\lambda_{1} \frac{\mathbf{E}_{3} \times \mathbf{q}}{\left|\mathbf{E}_{3} \times \mathbf{q}\right|}=\frac{\lambda_{1}}{\left|\mathbf{E}_{3} \times \mathbf{q}\right|}(-y, x, 0,-\alpha y, \alpha x, 0,-(\alpha+1) y,(\alpha+1) x, 0),
$$

donde

$$
\lambda_{1}=\frac{c_{3}}{\left|\mathbf{E}_{3} \times \mathbf{q}\right|}=\frac{\left[m_{1}+m_{2} \alpha^{2}+m_{3}(\alpha+1)^{2}\right]^{1 / 2}(x \dot{y}-y \dot{x})}{\left(x^{2}+y^{2}\right)^{1 / 2}}, \quad\left|\mathbf{E}_{3} \times \mathbf{q}\right|=\left(x^{2}+y^{2}\right)^{1 / 2} .
$$

En este caso, el vector $W_{1}$, vive en el subespacio $S_{\text {rot }}=\operatorname{span}\left\{\mathbf{E}_{3} \times \mathbf{r}\right\}$, por lo cual su dimensión es uno, $\operatorname{dim}\left(S_{\text {rot }}\right)=1$.

Tengamos en cuenta lo siguiente, considerar que las tres partículas tienen movimiento de rotación cambia la primera expresión encontrada para el escalar $\lambda_{3}$, es decir, ahora el vector de velocidad del sistema se descompone como

$$
\mathbf{v}=\lambda_{1} \frac{\mathbf{E}_{3} \times \mathbf{q}}{\left|\mathbf{E}_{3} \times \mathbf{q}\right|}+\lambda_{2} \frac{\mathbf{q}}{|\mathbf{q}|}+\lambda_{3} \frac{W_{3}}{\left|W_{3}\right|},
$$

por lo cual,

$$
\langle\mathbf{v}, \mathbf{v}\rangle=\lambda_{1}^{2}+\lambda_{2}^{2}+\lambda_{3}^{2}=2(U+h),
$$

de aquí, cuando las partículas $\mathbf{q}_{1}, \mathbf{q}_{2}$ y $\mathbf{q}_{3}$ están rotando en el plano $x-y$ al rededor de un vector en dirección del eje $z$, resulta que la velocidad configuracional es

$$
\bar{W}_{3}=\lambda_{3} \frac{W_{3}}{\left|W_{3}\right|}, \quad \text { con } \quad \lambda_{3}^{2}=2(U+h)-\left(\lambda_{1}^{2}+\lambda_{2}^{2}\right) .
$$

Por lo tanto, considerar movimiento de rotación en las partículas, afecta el cálculo de la tercer componente de la velocidad, $W_{3}$, esto nos hace ver que la tercer componente de la velocidad es complicada, a diferencia de las dos primeras componentes, $W_{1}$ y $W_{2}$. 


\subsection{Nuevas coordenadas para problemas de $N$-cuerpos en el plano}

En este capítulo, se presentan nuevas coordenadas que definen otro sistema de coordenadas para el problema de los $N$-cuerpos en el plano, las cuales son llamadas coordenadas de Fujiwara [2], y que son muy útiles para describir la forma de la configuración de sistemas de los $N$-cuerpos.

\subsection{Coordenadas de Fujiwara.}

Se usarán variables complejas para definir las nuevas coordenadas en $\mathbb{R}^{2}$. Para $\left(a_{x}, a_{y}\right)$, $\left(b_{x}, b_{y}\right) \in \mathbb{R}^{2}$, escribimos $a=a_{x}+i a_{y}, b=b_{x}+i b_{y} \in \mathbb{C}$.

Usamos las siguientes notaciones:

$$
\begin{aligned}
\bar{a} & =a_{x}-i b_{y}, \quad \text { complejo conjugado, } \\
a \cdot b & =a_{x} b_{x}+a_{y} b_{y}, \quad \text { producto interno, } \\
a \wedge b & =a_{x} b_{y}-a_{y} b_{x}, \quad \text { producto cruz. }
\end{aligned}
$$

Entonces tenemos,

$$
\bar{a} b=a \cdot b+i a \wedge b .
$$

Ahora denotaremos por $\mathbf{q}=\left(\mathbf{q}_{1}, \mathbf{q}_{2}, \ldots, \mathbf{q}_{N}\right)$ la posición de las $N$-partículas sobre el plano, por $m_{k}$ la masa de la $k$-ésima partícula y por $\mathbf{v}_{k}$ la velocidad de la partícula $k$. Las coordenadas de Fujiwara, $\mathbf{Q}_{k}$, están definidas por

$$
\mathbf{Q}_{k}=\exp \left(-i \mathbf{c} \int_{0}^{t} \frac{d t}{I}\right) \frac{\mathbf{q}_{k}}{\sqrt{I}}
$$

Las coordenadas de Fujiwara son válidas únicamente para partículas situadas en el plano, entonces notemos que en relación a las coordenadas $\mathbf{q}_{k}$ el sistema de partículas está girando en conjunto con una velocidad angular $\frac{\mathbf{c}}{I(\mathbf{q})}$. Además, como las partículas están restringidas a tener movimiento de rotación en el plano, sabemos que el momento angular es $\mathbf{c}=\left(0,0, c_{3}\right)$, por lo cual en coordenadas de Fujiwara el momento angular corresponde a la tercer coordenada de éste, es decir, $\mathbf{c}=c_{3}$.

En las coordenadas de Fujiwara, el factor fase $\exp \left(-i \mathbf{c} \int_{0}^{t} \frac{d t}{I}\right)$ es elegido tal que un observador situado en estas coordenadas rota con el sistema a la misma velocidad angular $\frac{\mathbf{c}}{I(\mathbf{q})}$. Así para el observador la velocidad angular parece ser cero, es decir, el momento angular en coordenadas de Fujiwara es cero, haciendo que estas coordenadas sean independientes del momento angular.

Ahora con el propósito de mostrar que el momento angular en términos de estas nuevas coordenadas es cero, usamos el resultado de la ecuación (4.14), así

$$
\sum_{k=1}^{N} m_{k} \overline{\mathbf{Q}}_{k} \frac{d \mathbf{Q}_{k}}{d t}=\sum_{k=1}^{N} m_{k} \mathbf{Q}_{k} \cdot \frac{d \mathbf{Q}_{k}}{d t}+i \sum_{k=1}^{N} m_{k} \mathbf{Q}_{k} \wedge \frac{d \mathbf{Q}_{k}}{d t}
$$


la parte imaginaria de la ecuación (4.16) corresponde al momento angular,

$$
\mathbf{c}\left(\mathbf{Q}_{k}\right)=\sum_{k=1}^{N} m_{k} \mathbf{Q}_{k} \wedge \frac{d \mathbf{Q}_{k}}{d t},
$$

sustituyendo la ecuación (4.15) en la anterior, y utilizando el hecho de que el centro de masa permanece fijo en el origen, es decir, $\sum_{k=1}^{N} m_{k} \mathbf{q}_{k}=0$, se obtiene que el momento angular en términos de las coordenadas de Fujiwara es

$$
\mathbf{c}\left(\mathbf{Q}_{k}\right)=\left(\frac{e^{-i \mathbf{c} \int_{0}^{t} \frac{d t}{I}}}{\sqrt{I}}\right) \sum_{k=1}^{N} m_{k} \mathbf{q}_{k} \wedge \frac{d \mathbf{Q}_{k}}{d t}=0
$$

De la misma forma se obtiene que la parte real de la ecuación (4.16)

$$
\sum_{k=1}^{N} m_{k} \mathbf{Q}_{k} \cdot \frac{d \mathbf{Q}_{k}}{d t}=0
$$

por lo tanto hemos obtenido

$$
\sum_{k=1}^{N} m_{k} \overline{\mathbf{Q}}_{k} \frac{d \mathbf{Q}_{k}}{d t}=0+i 0=0 .
$$

Además el momento angular en términos de las coordenadas de Fujiwara, resulta ser

$$
\begin{aligned}
\left|\mathbf{Q}_{k}\right|^{2} & =\left|\frac{\mathbf{q}_{k}}{\sqrt{I}}\right|^{2}\left|e^{-i \mathbf{c} \int_{0}^{t} \frac{d t}{I}}\right|^{2} \\
& =\frac{\left|\mathbf{q}_{k}\right|^{2}}{I}\left|\cos \left(\mathbf{c} \int_{0}^{t} \frac{d t}{I}\right)-i \operatorname{sen}\left(\mathbf{c} \int_{0}^{t} \frac{d t}{I}\right)\right|^{2}=\frac{\left|\mathbf{q}_{k}\right|^{2}}{I},
\end{aligned}
$$

por lo tanto,

$$
I\left(\mathbf{Q}_{k}\right)=\sum_{k=1}^{N} m_{k}\left|\mathbf{Q}_{k}\right|^{2}=\frac{1}{I} \sum_{k=1}^{N} m_{k}\left|\mathbf{q}_{k}\right|^{2}=1 .
$$

Es decir, el momento de inercia en términos de las coordenadas de Fujiwara permanece constante.

Luego, el factor de escala $\frac{1}{\sqrt{I(\mathbf{q})}}$ manda las coordenadas $\mathbf{q}_{k}$ dentro del elipsoide de masas, haciendo que cualquier problema que se estudie dentro del elipsoide de masas sea independiente de la escala, es decir, $\frac{1}{\sqrt{I(\mathbf{q})}}$ hace a las coordenadas de Fujiwara $\mathbf{Q}_{k}$ independientes del tamaño del sistema.

Entonces, si las coordenadas de Fujiwara son independientes tanto del tamaño del sistema como del momento angular, esto nos hace esperar que la evolución del sistema de partículas expresado en coordenadas de Fujiwara, $\mathbf{Q}_{k}$, describe el cambio en la forma de la configuración dada por las variables originales $\mathbf{q}_{k}$, de hecho estas soluciones en coordenadas de Fujiwara se caracterizan por el siguiente teorema. 
Teorema 4.1 Una solución en el plano expresada en coordenadas de $\mathbf{q}_{k}(t)$ es homográfica si y sólo si $\frac{d}{d t} \mathbf{Q}_{k}(t)=0$ para cada $k$ y todo tiempo $t$.

Demostración:

Supongamos que

$$
\mathbf{q}_{k}(t)=R(t) e^{i \theta(t)} \mathbf{q}_{k}(0)
$$

es una solución homográfica, donde $R(t)>0$ describe la evolución del sistema de partículas en tamaño y $\theta \in \mathbb{R}$ describe sus rotaciones. Sin pérdida de generalidad podemos tomar como condiciones iniciales $R(0)=1$ y $\theta(0)=0$. Entonces

$$
I(t)=\sum_{k=1}^{N} m_{k}\left|\mathbf{q}_{k}\right|^{2}=\sum_{k=1}^{N} m_{k}|R(t)|^{2}\left|\mathbf{q}_{k}(0)\right|^{2}=R^{2}(t) \sum_{k=1}^{N} m_{k}\left|\mathbf{q}_{k}(0)\right|^{2}=R^{2}(t) I(0)
$$

y el momento angular del sistema de partículas en el plano es

$$
\mathbf{c}(t)=I(t) \frac{d \theta}{d t}=R^{2}(t) I(0) \frac{d \theta}{d t},
$$

de donde $\frac{d \theta}{d t}=\frac{\mathbf{c}}{I(t)}$, e integrando de 0 a $t$ se obtiene

$$
\theta=\mathbf{c} \int_{0}^{t} \frac{d t}{I(t)}
$$

Entonces las variables de Fujiwara toman la forma

$$
\mathbf{Q}_{k}=e^{-i \mathbf{c} \int_{0}^{t} \frac{d t}{I}} \frac{\mathbf{q}_{k}}{\sqrt{I}}=e^{-i \theta(t)} \frac{\mathbf{q}_{k}(t)}{R(t) \sqrt{I(0)}}=\frac{\mathbf{q}_{k}(0)}{\sqrt{I(0)}}
$$

lo cual es una constante y por lo tanto

$$
\frac{d}{d t} \mathbf{Q}_{k}(t)=0
$$

Ahora probaremos el inverso. Supongamos que $\frac{d}{d t} \mathbf{Q}_{k}(t)=0$ entonces

$$
\mathbf{Q}_{k}(t)=\mathbf{Q}_{k}(0)
$$

Luego en variables de Fujiwara $\mathbf{q}_{k}(t)$ tienen la forma

$$
\mathbf{q}_{k}(t)=\sqrt{I} e^{-i \mathbf{c} \int_{0}^{t} \frac{d t}{I}} \mathbf{Q}_{k}(t)=\sqrt{I} e^{-i \mathbf{c} \int_{0}^{t} \frac{d t}{I}} \mathbf{Q}_{k}(0), \quad \text { para cada } k \text { y para todo } t,
$$

lo cual implica que las soluciones son homográficas.

Otro aspecto importante e interesante de las coordenadas de Fujiwara, es que están relacionadas con la descomposición de la velocidad de Saari, como veremos a continuación. 
Tenemos

$$
\begin{aligned}
\frac{d}{d t} \mathbf{Q}_{k}(t) & =\frac{e^{-i \mathbf{c} \int_{0}^{t} \frac{d t}{I}}}{\sqrt{I}} \frac{d \mathbf{q}_{k}}{d t}+\frac{\mathbf{q}_{k}}{\sqrt{I}} e^{-i \mathbf{c} \int_{0}^{t} \frac{d t}{I}}\left(\frac{-i \mathbf{c}}{I}\right)+e^{-i \mathbf{c} \int_{0}^{t} \frac{d t}{I}} \mathbf{q}_{k}\left(-\frac{1}{2 \sqrt{I} I} \frac{d I}{d t}\right) \\
& =\frac{e^{-i \mathbf{c} \int_{0}^{t} \frac{d t}{I}}}{\sqrt{I}}\left[\frac{d \mathbf{q}_{k}}{d t}-\left(\frac{1}{2 I} \frac{d I}{d t}+i \frac{\mathbf{c}}{I}\right) \mathbf{q}_{k}\right],
\end{aligned}
$$

despejando de ésta la velocidad del sistema, $\frac{d \mathbf{q}_{k}}{d t}$, y haciendo $\frac{d I}{d t}=\dot{I}$ obtenemos

$$
\begin{aligned}
\mathbf{v}_{k} & =\frac{d \mathbf{q}_{k}}{d t}=\dot{\mathbf{q}}_{k}=\left[\frac{d}{d t} \mathbf{Q}_{k}(t)-\frac{e^{-i \mathbf{c} \int_{0}^{t} \frac{d t}{I}}}{\sqrt{I}}\left(\frac{1}{2 I} \frac{d I}{d t}+i \frac{\mathbf{c}}{I}\right) \mathbf{q}_{k}\right] \frac{\sqrt{I}}{e^{-i \mathbf{c} \int_{0}^{t} \frac{d t}{T}}} \\
& =\sqrt{I} e^{i \mathbf{c} \int_{0}^{t} \frac{d t}{I}} \frac{d \mathbf{Q}_{k}}{d t}+\frac{\dot{I}}{2 I} \mathbf{q}_{k}+i \frac{\mathbf{c}}{I} \mathbf{q}_{k} .
\end{aligned}
$$

Si ahora despejamos $\mathbf{q}_{k}$ de la ecuación (4.15), la cual define las coordenadas de Fujiwara, se tiene

$$
\mathbf{q}_{k}=\sqrt{I} e^{i \mathbf{c} \int_{0}^{t} \frac{d t}{I}} \mathbf{Q}_{k},
$$

por último sustituyendo ésta en la última igualdad de la ecuación (4.17), obtenemos la descomposición de la velocidad en términos de las coordenadas de Fujiwara,

$$
\mathbf{v}_{k}=\sqrt{I} e^{i \mathbf{c} \int_{0}^{t} \frac{d t}{I}}\left[\frac{d \mathbf{Q}_{k}}{d t}+\frac{\dot{I}}{2} \frac{\mathbf{Q}_{k}}{I}+i \mathbf{c} \frac{\mathbf{Q}_{k}}{I}\right]
$$

puesto que, el primer término el cual corresponde a $\frac{d \mathbf{Q}_{k}}{d t}$, describe el cambio en la forma de la configuración, por lo cual está es la componente de la velocidad que describe el cambio en la configuración,

$$
W_{3}(\mathbf{Q})=\sqrt{I} e^{i \mathbf{c} \int_{0}^{t} \frac{d t}{I}} \frac{d \mathbf{Q}_{k}}{d t} .
$$

El segundo término caracterizado por la expresión $\frac{\dot{I}}{2}$, determina la razón de cambio en el tamaño del sistema por lo cual éste corresponde a la componente de la velocidad dedicada al cambio escalar,

$$
W_{2}(\mathbf{Q})=\sqrt{I} e^{i \mathbf{c} \int_{0}^{t} \frac{d t}{I}} \frac{\dot{I}}{2} \frac{\mathbf{Q}_{k}}{I}
$$

y el tercer término correspondiente a la parte imaginaria, está caracterizado por el momento angular c, por lo cual éste corresponde a la componente de la velocidad dedicada a las rotaciones del sistema vistas como un cuerpo rígido, es decir,

$$
W_{1}(\mathbf{Q})=i \sqrt{I} e^{i \mathbf{c} \int_{0}^{t} \frac{d t}{I}} \mathbf{c} \frac{\mathbf{Q}_{k}}{I} .
$$

Por lo tanto con la ecuación (4.18) se tiene una expresión explícita de la descomposición de la velocidad de Saari, en términos de las coordenadas de Fujiwara,

$$
\mathbf{v}(\mathbf{Q})=W_{1}(\mathbf{Q})+W_{2}(\mathbf{Q})+W_{3}(\mathbf{Q}) .
$$

En la siguiente sección, aplicaremos las coordenadas de Fujiwara al problema colineal de tres cuerpos en el plano, con el propósito de obtener la descomposición de la velocidad de Saari en términos de las coordenadas de Fujiwara. 


\subsubsection{Problema colineal de 3-cuerpos en coordenadas de Fujiwara}

Sabemos que las coordenadas de Fujiwara sólo pueden ser utilizadas en problemas de los $N$-cuerpos situados en el plano, lo cual nos permite aplicar éstas al problema colineal de 3-cuerpos en el plano. Anteriormente obtuvimos la descomposición de la velocidad de Saari para el problema de tres cuerpos en el plano, sin embargo, ahora nuestro interés es obtener la descomposición de la velocidad de dicho problema en términos de las coordenadas de Fujiwara.

Consideremos nuevamente tres partículas con posiciones $\mathbf{q}_{1}, \mathbf{q}_{2}, \mathbf{q}_{3}$ y con masas distintas $m_{1}, m_{2}$ y $m_{3}$, respectivamente, las cuales se encuentran colocadas de tal forma que siempre están sobre una linea recta en el plano $x-y$ que pasa por el origen, el cual es el centro de masa, expresando de nuevo la posición de estas partículas por

$$
\begin{aligned}
& \mathbf{q}_{1}=\mathbf{r}_{2}-\mathbf{r}_{1}=(x, y, 0), \\
& \mathbf{q}_{2}=\alpha\left(\mathbf{r}_{2}-\mathbf{r}_{1}\right)=\alpha(x, y, 0) \quad \text { donde } \quad \alpha \in \mathbb{R},
\end{aligned}
$$

además, en este análisis basta considerar las dos primeras posiciones, puesto que la tercera se encuentra en términos de las dos primeras.

Consideraremos dos casos en este problema:

- El primero caso consiste en suponer que las partículas tienen movimiento de rotación, por lo tanto éstas se encuentran girando al rededor de un vector perpendicular al plano $x-y$. Además, sabemos que la descomposición de la velocidad en términos de las coordenadas de Fujiwara, está dada por

$$
\mathbf{v}_{k}=\frac{d \mathbf{q}_{k}}{d t}=\sqrt{I} e^{i \mathbf{c} \int_{0}^{t} \frac{d t}{I}}\left[\frac{d \mathbf{Q}_{k}}{d t}+\frac{\dot{I}}{2} \frac{\mathbf{Q}_{k}}{I}+i \mathbf{c} \frac{\mathbf{Q}_{k}}{I}\right]
$$

donde

$$
\frac{d}{d t} \mathbf{Q}_{k}(t)=\frac{e^{-i \mathbf{c} \int_{0}^{t} \frac{d t}{I}}}{\sqrt{I}}\left[\frac{d \mathbf{q}_{k}}{d t}-\left(\frac{1}{2 I} \frac{d I}{d t}+i \frac{\mathbf{c}}{I}\right) \mathbf{q}_{k}\right]
$$

Para el problema colineal de tres cuerpos en el plano con rotación, podemos escribir el vector de velocidad del sistema, como sigue

$$
\begin{aligned}
\mathbf{v} & =\left(\begin{array}{c}
\mathbf{v}_{1} \\
\mathbf{v}_{2}
\end{array}\right)=W_{3}(\mathbf{Q})+W_{2}(\mathbf{Q})+W_{1}(\mathbf{Q}) \\
& =\sqrt{I} e^{i \mathbf{c} \int_{0}^{t} \frac{d t}{I}}\left(\begin{array}{c}
\frac{d \mathbf{Q}_{1}}{d t} \\
\frac{d \mathbf{Q}_{2}}{d t}
\end{array}\right)+\sqrt{I} e^{i \mathbf{c} \int_{0}^{t} \frac{d t}{I}}\left(\begin{array}{c}
\frac{\dot{I}}{2} \frac{\mathbf{Q}_{1}}{I} \\
\frac{\dot{I}}{2} \frac{\mathbf{Q}_{2}}{I}
\end{array}\right)+\sqrt{I} e^{i \mathbf{c} \int_{0}^{t} \frac{d t}{I}}\left(\begin{array}{c}
i \mathbf{c} \frac{\mathbf{Q}_{1}}{I} \\
i \mathbf{c} \frac{\mathbf{Q}_{2}}{I}
\end{array}\right) .
\end{aligned}
$$


Luego, haciendo algunos cálculos se llega

$$
\begin{aligned}
& I=\left[m_{1}+m_{2} \alpha^{2}+m_{3}(\alpha+1)^{2}\right]\left(x^{2}+y^{2}\right), \\
& \dot{I}=2\left[m_{1}+m_{2} \alpha^{2}+m_{3}(\alpha+1)^{2}\right](x \dot{x}+y \dot{y}), \\
& \mathbf{c}=c_{3}=\left[m_{1}+m_{2} \alpha^{2}+m_{3}(\alpha+1)^{2}\right](x \dot{y}-y \dot{x}) .
\end{aligned}
$$

Por lo tanto, obtenemos que las tres componentes de la descomposición de la velocidad de Saari, en términos de las coordenadas de Fujiwara para el problema colineal de tres cuerpos en el plano, con movimiento de rotación son

$$
\begin{aligned}
\mathbf{v} & =W_{1}(\mathbf{Q})+W_{2}(\mathbf{Q})+W_{3}(\mathbf{Q}) \\
& =\sqrt{I} e^{i \mathbf{c} \int_{0}^{t} \frac{d t}{I}}\left(\begin{array}{c}
i \frac{x \dot{y}-y \dot{x}}{x^{2}+y^{2}} \\
\mathbf{Q}_{1} \\
i \frac{x \dot{y}-y \dot{x}}{x^{2}+y^{2}}
\end{array}\right)+\sqrt{I} \mathbf{Q}_{2} e^{i \mathbf{c} \int_{0}^{t} \frac{d t}{I}}\left(\begin{array}{c}
\frac{x \dot{x}+y \dot{y}}{x^{2}+y^{2}} \mathbf{Q}_{1} \\
\frac{x \dot{x}+y \dot{y}}{x^{2}+y^{2}} \mathbf{Q}_{2}
\end{array}\right)+ \\
& +\left(\begin{array}{c}
\dot{\mathbf{q}}_{1}-[(x \dot{x}+y \dot{y})+i(x \dot{y}-y \dot{x})] \frac{\mathbf{q}_{1}}{x^{2}+y^{2}} \\
\dot{\mathbf{q}}_{2}-[(x \dot{x}+y \dot{y})+i(x \dot{y}-y \dot{x})] \frac{\mathbf{q}_{2}}{x^{2}+y^{2}}
\end{array}\right)
\end{aligned}
$$

- El segundo caso consiste en considerar que las partículas no presenta movimiento de rotación, por lo cual están restringidas a permanecer sobre la misma linea recta para todo tiempo t. Por lo cual, en este caso el momento angular es nulo, $\mathbf{c}=c_{3}=0$, obteniendo

$$
W_{1}(\mathbf{Q})=i \sqrt{I} e^{i \mathbf{c} \int_{0}^{t} \frac{d t}{I}} \mathbf{c} \frac{\mathbf{Q}_{k}}{I}=0
$$

y haciendo que la descomposición de la velocidad en términos de las coordenadas de Fujiwara se reduzca a tener

$$
\begin{aligned}
\mathbf{v} & =W_{2}(\mathbf{Q})+W_{3}(\mathbf{Q}) \\
& =\sqrt{I}\left(\begin{array}{c}
\frac{x \dot{x}+y \dot{y}}{x^{2}+y^{2}} \\
\mathbf{Q}_{1} \\
\frac{x \dot{x}+y \dot{y}}{x^{2}+y^{2}}
\end{array}\right)+\left(\begin{array}{c}
\mathbf{q}_{1}-(x \dot{x}+y \dot{y}) \frac{\mathbf{q}_{1}}{x^{2}+y^{2}} \\
\dot{\mathbf{q}}_{2}-(x \dot{x}+y \dot{y}) \frac{\mathbf{q}_{2}}{x^{2}+y^{2}}
\end{array}\right) .
\end{aligned}
$$

Finalmente, al comparar las expresiones obtenidas para los vectores $W_{1}(\mathbf{Q})$ y $W_{2}(\mathbf{Q})$, con las primeras expresiones conseguidas para $W_{1}(\mathbf{q}, \mathbf{c})$ y $W_{2}(\mathbf{q}, I)$, respectivamente, podemos observar que estas componentes en términos de las coordenadas de Fujiwara contienen prácticamente los mismos términos. Sin embargo, resulta más complicado hacer comparaciones con la tercer componente $W_{3}(\mathbf{Q})$ y la conseguida anteriormente para el problema colineal 
de tres cuerpos en el plano, $W_{3}$, lo cual nos hace ver nuevamente lo complicada que es la componente dedicada al cambio en la forma de la configuración. Pero nos atrevemos a decir que la descomposición de la velocidad en términos de las coordenadas de Fujiwara, permite obtener resultados similares a los que se pudieran obtener en problemas de los $N$-cuerpos en el plano, utilizando la descomposición de la velocidad original. 


\section{CAPÍTULO 5}

\section{Aplicaciones}

Hasta aquí ya sabemos en que consiste la descomposición de la velocidad de Saari, y dimos una nueva forma de clasificar problemas de los $N$-cuerpos en términos de ésta, también dimos ejemplos de esta nueva clasificación y realizamos la descomposición del correspondiente vector de velocidad de los tres sistemas uno-dimensionales presentados. Ahora estamos interesados en usar la descomposición de la velocidad de Saari, de dos de los sistemas uno-dimensionales estudiados: el problema romboidal generalizado en rotación y el problema isósceles en rotación. Usando la descomposición de la velocidad estudiaremos lo que en física se conoce como el potencial aumentado, lo cual coincidirá exactamente con la primer componente de nuestra descomposición. Nuestro objetivo es relacionar las constantes de movimiento, integral de energía total y momento angular con la intención de analizar aspectos cualitativos relacionados con el escape de las partículas, haciendo una generalización de las regiones de Hill, para ello utilizaremos algunos de los conceptos ya conocidos y estudiaremos los movimientos que se producen. Entonces, como una aplicación importante de la descomposición de la velocidad, encontraremos una relación entre las constantes de movimiento, integral total y momento angular, obteniendo así una generalización de las regiones de Hill.

\subsection{Dinámica del problema romboidal generalizado en rotación}

Supongamos que las partículas de masa $m_{1}$ y $m_{2}$ al tiempo $t$, se encuentran a una distancia $\rho_{1}$ del origen, y a una distancia $\rho_{2}$ sobre el eje $z$ al origen, respectivamente. Entonces la función potencial dada por la ecuación (4.7) se puede escribir como

$$
U=\frac{m_{2}^{2}}{2 \rho_{2}}+\frac{2 N m_{1} m_{2}}{\left(\rho_{1}^{2}+\rho_{2}^{2}\right)^{1 / 2}}+\sum_{k<j \leq N} \frac{m_{1}^{2}}{\left|\mathbf{r}_{j}-\mathbf{r}_{k}\right|}
$$


Denotemos por $\phi$ (al mismo tiempo $t$ ), al ángulo de inclinación de la línea recta en el semiplano $z>0$, que contiene a una partícula del $N$-polígono regular y la partícula sobre el eje $z$ (véase la figura 5.1), expresando $\left|\mathbf{r}_{j}-\mathbf{r}_{k}\right|$ en términos de la posición de las partículas sobre el plano $x-y$, es decir, por la ecuación (3.2) se tiene que

$$
\mathbf{r}_{j}=\rho_{1} \exp \left(j \frac{2 \pi}{N}+\theta i\right) \quad \text { y } \quad \mathbf{r}_{k}=\rho_{1} \exp \left(k \frac{2 \pi}{N}+\theta i\right)
$$

de aquí

$$
\begin{aligned}
\left|\mathbf{r}_{j}-\mathbf{r}_{k}\right| & =\rho_{1}\left|e^{\left(j \frac{2 \pi}{N}\right)} e^{\theta i}-e^{\left(k \frac{2 \pi}{N}\right)} e^{\theta i}\right|=\rho_{1}\left|e^{\left(j \frac{2 \pi}{N}\right)}-e^{\left(k \frac{2 \pi}{N}\right)}\right| \\
& =\rho_{1}\left|\cos \left(\frac{2 \pi}{N} j\right)-\cos \left(\frac{2 \pi}{N} k\right)+i\left(\operatorname{sen}\left(\frac{2 \pi}{N} j\right)-\operatorname{sen}\left(\frac{2 \pi}{N} k\right)\right)\right| \\
& =\rho_{1} \sqrt{-2\left(\cos \left(\frac{2 \pi}{N} j\right) \cos \left(\frac{2 \pi}{N} k\right)+\operatorname{sen}\left(\frac{2 \pi}{N} j\right) \operatorname{sen}\left(\frac{2 \pi}{N} k\right)\right)+2} \\
& =\rho_{1} \sqrt{-2 \cos \left(\frac{2 \pi}{N}(j-k)\right)+2}=\rho_{1} \sqrt{-2\left(1-2 \operatorname{sen}^{2} \frac{\pi}{N}(j-k)\right)+2} \\
& =\rho_{1} \sqrt{4 \operatorname{sen}^{2}\left(\frac{\pi}{N}(j-k)\right)}=2 \rho_{1} \operatorname{sen}\left(\frac{\pi}{N}(j-k)\right),
\end{aligned}
$$

por lo cual, la función potencial toma la forma

$$
U=\frac{1}{\rho_{1}}\left(\frac{m_{2}^{2}}{2 \tan \phi}+\frac{2 N m_{1} m_{2}}{\sec \phi}+\sum_{k<j \leq N} \frac{m_{1}^{2}}{2 \operatorname{sen}\left(\frac{\pi}{N}(j-k)\right)}\right), \quad \phi \in(0, \pi / 2) .
$$
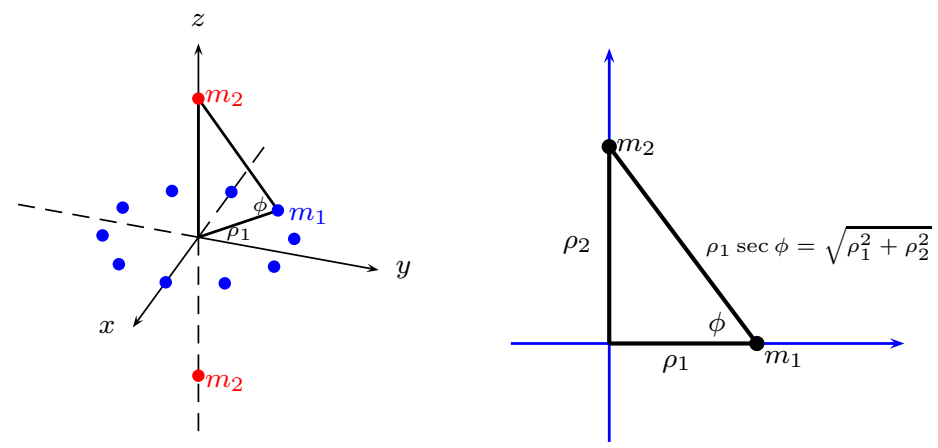

Figura 5.1: Ángulo de inclinación $\phi$, del cual se tiene $\tan \phi=\frac{\rho_{2}}{\rho_{1}}$.

El ángulo $\phi$ lo consideramos distinto de cero para evitar la colisión binaria de las partículas que se encuentran sobre el eje $z$, también es importante mencionar que las $N$ partículas en el plano $x$ - $y$ tampoco presentan colisión total, ya que el momento angular es distinto de cero.

Anteriormente usando la descomposición de la velocidad, observamos que la integral de energía $\langle\mathbf{v}, \mathbf{v}\rangle=2(U+h)$, puede ser escrita en la forma

$$
\lambda_{1}^{2}+\lambda_{2}^{2}+\lambda_{3}^{2}=2(U+h),
$$


por lo cual, ésta siempre cumple la siguiente desigualdad

$$
2(U+h)-\lambda_{1}^{2}=\lambda_{2}^{2}+\lambda_{3}^{2} \geq 0 .
$$

Por otro lado, sabemos del capítulo anterior que para el problema romboidal generalizado en rotación

$$
\lambda_{1}=\frac{\mathbf{c}}{\left(N m_{1} \rho^{2}\right)^{1 / 2}},
$$

por lo cual, la desigualdad (5.3) en este caso toma la forma

$$
2(U+h)-\frac{\mathbf{c}^{2}}{N m_{1} \rho_{1}^{2}} \geq 0,
$$

donde el lado izquierdo de esta última desigualdad es conocido en física como el potencial aumentado, es decir, el potencial clásico más la acción del momento angular, lo cual coincide exactamente con la primer componente de nuestra descomposición de la velocidad, $W_{1}$, así hemos encontrado una relación entre las constantes de movimiento; integral de energía total y momento angular con el escape de partículas, ya que hemos obtenido de la ecuación (5.4) una función que se encuentra en términos de las distancias mutuas entre partículas, el nivel de energía $h$ en el que nos encontremos y el momento angular, es decir, hemos obtenido la generalización de las regiones de Hill, que a diferencia de las regiones de Hill tradicionales, en las generalizadas se introduce el momento angular como tercer parámetro, el cual es necesario para describir la dinámica del problema, ya que en este sistema las partículas sobre el plano $x-y$ tienen movimiento de rotación al rededor del eje $z$.

Por tanto, la función dada por la ecuación (5.4) implica tener la siguiente restricción en la dinámica del problema romboidal generalizado en rotación

$$
\frac{\mathbf{c}^{2}}{N m_{1} \rho_{1}^{2}} \leq 2(U+h)
$$

considerando la dinámica de las partículas en la frontera, es decir, ahora estudiaremos la siguiente igualdad

$$
\frac{\mathbf{c}^{2}}{2 N m_{1} \rho_{1}^{2}}=U+h
$$

Sustituyendo la función potencial dada por la ecuación (5.2) en la igualdad anterior, se obtiene la siguiente expresión

$$
\frac{\mathbf{c}^{2}}{2 N m_{1} \rho_{1}^{2}}-\frac{1}{\rho_{1}}\left(\frac{m_{2}^{2}}{2 \tan \phi}+\frac{2 N m_{1} m_{2}}{\sec \phi}+\sum_{k<j \leq N} \frac{m_{1}^{2}}{2 \operatorname{sen}\left(\frac{\pi}{N}(j-k)\right)}\right)-h=0 .
$$

Multiplicando por $-\rho_{1}^{2}$ la ecuación anterior, y nombrando

$$
\left(\frac{m_{2}^{2}}{2 \tan \phi}+\frac{2 N m_{1} m_{2}}{\sec \phi}+\sum_{k<j \leq N} \frac{m_{1}^{2}}{2 \operatorname{sen}\left(\frac{\pi}{N}(j-k)\right)}\right)=F(\phi)
$$


ésta se convierte en un polinomio cuadrático, el cual denotamos de la siguiente manera

$$
P\left(\rho_{1}\right)=h \rho_{1}^{2}+F(\phi) \rho_{1}-\frac{\mathbf{c}^{2}}{2 N m_{1}} .
$$

De esta forma la desigualdad de la ecuación (5.5), puede ser escrita como

$$
\frac{\mathbf{c}^{2}}{2 N m_{1} \rho_{1}^{2}} \leq \frac{F(\phi)}{\rho_{1}}+h,
$$

con ésta y el polinomio $P\left(\rho_{1}\right)$ analizamos lo que ocurre al tomar valores positivos, negativos y cero, de la energía $h$.

(a) Si $h>0$, entonces

$$
h \rho_{1}^{2}+F(\phi) \rho_{1}-\frac{\mathbf{c}^{2}}{2 N m_{1}} \geq 0,
$$

lo cual implica que $\rho_{1}$ puede tomar los valores

$$
\rho_{1} \geq \frac{-F(\phi)+\sqrt{F^{2}(\phi)+\frac{2 \mathbf{c}^{2} h}{N m_{1}}}}{2 h}, \quad \forall \quad \phi \in(0, \pi / 2),
$$

es decir, las partículas de masa $m_{1}$ sobre el plano $x-y$ pueden escapar a infinito, lo mismo sucede con partículas de masa $m_{2}$, lo cual se ve de la relación

$$
\rho_{2}=\rho_{1} \tan \phi
$$

pues si $\rho_{1}$ y tan $\phi$ con $(0, \pi / 2)$ crecen, así lo hará $\rho_{2}$. La gráfica de $P\left(\rho_{1}\right)$ en este caso, es una parábola que abre hacia arriba como se muestra en la figura 5.2 (a).

(b) Si $h=0$, entonces por la relación de energía se tiene que $\rho_{1}$ puede tomar los valores

$$
\rho_{1} \geq \frac{\mathbf{c}^{2}}{2 N m_{1} F(\phi)}, \quad \forall \quad \phi \in(0, \pi / 2),
$$

es decir, nuevamente las partículas de masa $m_{1}$ sobre el plano $x-y$ pueden escapar a infinito. De la relación $\rho_{2}=\rho_{1} \tan \phi$ si $\rho_{1} \rightarrow \infty$ o tan $\phi \rightarrow \infty$, entonces $\rho_{2}$ de igual forma lo hará, por lo tanto las partículas sobre el eje $z$ también pueden escapar a infinito. En este segundo caso, la gráfica de $P\left(\rho_{1}\right)$ es una linea recta, la figura 5.2 (b) muestra la situación.

(c) Si $h<0$, entonces obtenemos la siguiente desigualdad

$$
-|h| \rho_{1}^{2}+F(\phi) \rho_{1}-\frac{\mathbf{c}^{2}}{2 N m_{1}} \geq 0,
$$

lo cual implica que los únicos valores que $\rho_{1}$ puede tomar para todo $\phi \in(0, \pi / 2)$, están comprendidos entre sus dos raíces, es decir, en el siguiente intervalo

$$
\left[\frac{-F(\phi)+\sqrt{F^{2}(\phi)-\frac{2 \mathbf{c}^{2}|h|}{N m_{1}}}}{-2|h|}, \frac{-F(\phi)-\sqrt{F^{2}(\phi)-\frac{2 \mathbf{c}^{2}|h|}{N m_{1}}}}{-2|h|}\right],
$$


entonces el movimiento de las partículas de masa $m_{1}$ es acotado, lo cual significa que las partículas nunca colisionan entre ellas, y no pueden escapar a infinito. La gráfica de $P(\rho)$ es una parábola que abre hacia abajo, la figura 5.2 (c) muestra la situación.

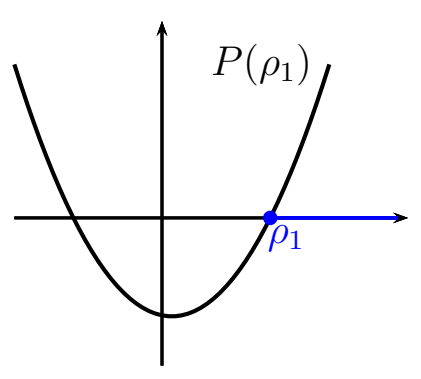

(a) $\rho_{1} \geq \frac{-F(\phi)+\sqrt{F^{2}(\phi)+\frac{2 c^{2} h}{N m_{1}}}}{2 h}$

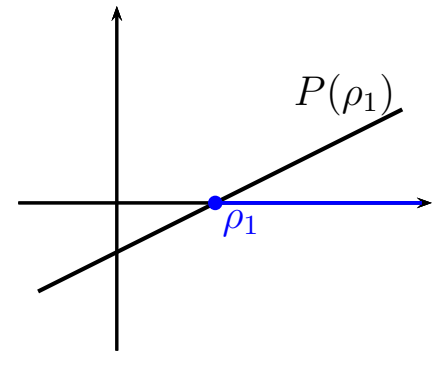

(b) $\rho_{1} \geq \frac{\mathrm{c}^{2}}{2 N m_{1} F(\phi)}$

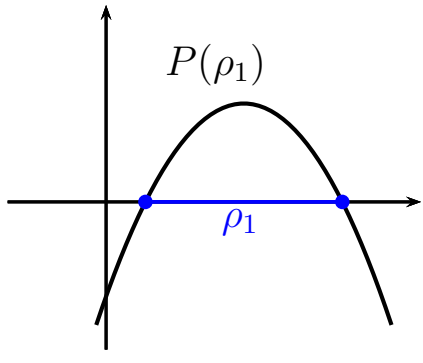

(c) $\rho_{1} \in\left[\frac{-F(\phi)+\sqrt{\Delta}}{-2|h|}, \frac{-F(\phi)-\sqrt{\Delta}}{-2|h|}\right]$

Figura 5.2: Gráficas de $P\left(\rho_{1}\right)$ del problema romboidal generalizado en rotación.

Resta saber en el tercer caso, qué es lo que sucede con las partículas que se encuentran sobre el eje $z$, ya que aunque $\rho_{1}$ se encuentra acotada, esto no implica que las partículas de masa $m_{2}$ no puedan escapar a infinito, para esto analicemos cómo se comporta la función $F(\phi)$.

Observemos que

$$
\lim _{\phi \rightarrow 0} F(\phi)=\infty \quad \text { y } \quad F^{\prime}(\phi)=-\frac{m_{2}^{2}}{2 \operatorname{sen}^{2} \phi}-2 N m_{1} m_{2} \operatorname{sen} \phi,
$$

entonces $F(\phi)>0$ y $F^{\prime}(\phi)<0$, es decir, $F(\phi)$ es positiva, no tiene puntos críticos y la pendiente en cada uno de sus puntos es negativa, por lo tanto $F(\phi)$ es decreciente, la interpretación geométrica se ve en la figura 5.3.

Las partículas sobre el eje $z$ pueden escapar a infinito y podemos calcular el valor de $\mathbf{c}|h|^{1 / 2}$ para el cual el escape de las partículas de masa $m_{2}$ es posible, entonces como el polinomio de la desigualdad (5.6) tiene raíces reales su discriminante debe cumplir

$$
\Delta=F^{2}(\phi)-\frac{2 \mathbf{c}^{2}|h|}{N m_{1}} \geq 0,
$$

entonces de aquí se tiene la desigualdad

$$
\mathbf{c}|h|^{1 / 2} \leq\left(N m_{1}\right)^{1 / 2} F(\phi), \quad \forall \phi \in(0, \pi / 2),
$$

luego, notemos que cuando $\phi$ va de 0 a $\pi / 2$, el último valor que $\mathbf{c}|h|^{1 / 2}$ puede alcanzar es cuando $\phi \rightarrow \pi / 2$, entonces

$$
\mathbf{c}|h|^{1 / 2}=\left(N m_{1}\right)^{1 / 2} \lim _{\phi \rightarrow \pi / 2} F(\phi)=\left(N m_{1}\right)^{1 / 2} \sum_{i<j \leq N} \frac{m_{1}^{2}}{2 \operatorname{sen}\left(\frac{\pi}{N}(j-k)\right)},
$$


donde $\lim _{\phi \rightarrow \pi / 2} F(\phi)=\sum_{i<j \leq N} \frac{m_{1}^{2}}{2 \operatorname{sen}\left(\frac{\pi}{N}(j-k)\right)}$, haciendo

$$
A=\left(N m_{1}\right)^{1 / 2} \sum_{i<j \leq N} \frac{m_{1}^{2}}{2 \operatorname{sen}\left(\frac{\pi}{N}(j-k)\right)}
$$

se obtienen los siguientes dos casos:

- Si $\mathbf{c}|h|^{1 / 2}>A$, entonces el movimiento de las partículas de masa $m_{2}$ es acotado (véase la figura 5.3), ya que el mínimo valor que puede alcanzar $\mathbf{c}|h|^{1 / 2}$ se tiene cuando hacemos tender $\phi \rightarrow \pi / 2$, pues en este análisis el ángulo $\phi$ no puede ser mayor o igual que $\pi / 2$.

- Si c $|h|^{1 / 2} \leq A$, entonces las partícula de masa $m_{2}$ escapan a infinito, (véase la figura $5.3)$.

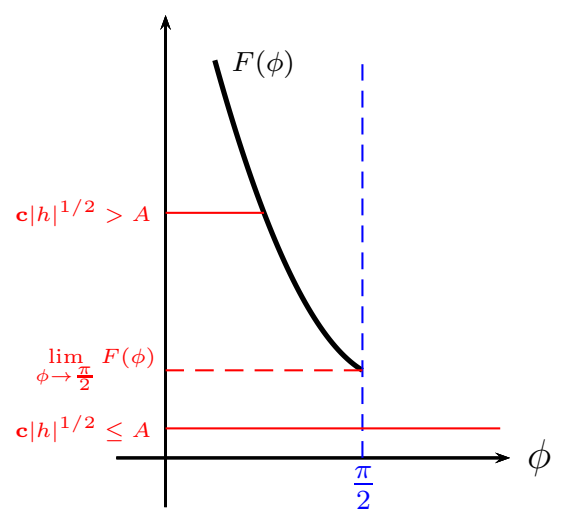

Figura 5.3: Gráfica de $F(\phi)$.

Observemos que en los dos casos anteriores el lado izquierdo de las desigualdades corresponde a lo que hemos visto como el límite inferior de la medida configuracional, $I^{1 / 2} U$ la cual mide cómo cambia la forma de la configuración, es decir, $I^{1 / 2} U$ está acotado inferiormente por $c|h|^{1 / 2}$ si y sólo si $I^{1 / 2}=\frac{c}{2|h|^{1 / 2}}$.

Por lo tanto, como aplicación importante de la descomposición de la velocidad, encontramos una relación entre las constantes de movimiento, integral total y momento angular con el escape de las partículas del problema romboidal generalizado en rotación.

\subsection{Dinámica del problema isósceles en rotación}

En lo que sigue, analizaremos la dinámica del problema isósceles en rotación, para ello supongamos que las partículas que se encuentran rotando al rededor del eje $z$ con masa $m_{1}$ están a una distancia $\mu_{1}$ del origen, y sea $\mu_{2}$ la distancia de la partícula de masa $m_{2}$ sobre el eje $z$ al origen. 
Así la función potencial dada por la ecuación (4.13) toma la forma

$$
U=\frac{m_{1}^{2}}{2 \mu_{1}}+\frac{2 m_{1} m_{2}}{\left(\mu_{1}^{2}+\mu_{2}^{2}\right)^{1 / 2}} .
$$

Sea $\varphi$ al ángulo de inclinación de la línea recta en el semiplano $z>0$, que contiene a una partícula sobre el plano $x$-y y la partícula sobre el eje $z$ de masa $m_{2}$ (la interpretación geométrica se observa en la figura 5.4) de aquí el potencial de la ecuación (5.7) se escribe como

$$
U=\frac{1}{\mu_{1}}\left(\frac{m_{1}^{2}}{2}+\frac{2 m_{1} m_{2}}{\sec \varphi}\right) \quad \text { para todo } \varphi \in[0, \pi / 2) .
$$

En este caso, el ángulo $\varphi$ puede tomar el valor cero, ya que aquí no existe colisión binaria, además las partículas sobre el plano $x$ - $y$ tampoco presenta colisión, puesto que el momento angular es distinto de cero.
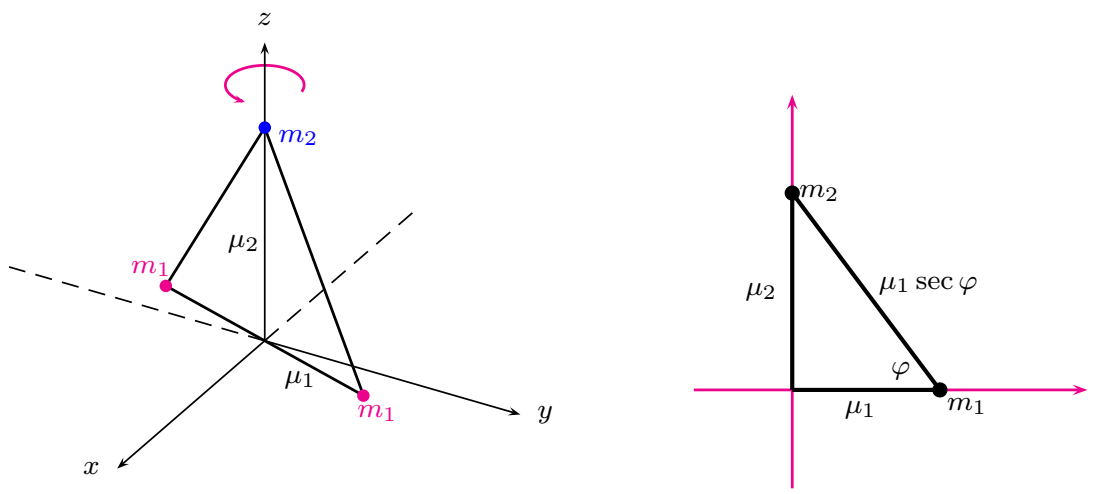

Figura 5.4: Ángulo de inclinación $\varphi$, del cual se tiene $\tan \varphi=\frac{\mu_{2}}{\mu_{1}}$.

Utilizando la descomposición de la velocidad del problema isósceles en rotación, obtenemos de la integral de energía total la siguiente desigualdad

$$
2(U+h)-\lambda_{1}^{2} \geq 0
$$

donde la correspondiente $\lambda_{1}$ para el problema isósceles es

$$
\lambda_{1}=\frac{\mathbf{c}}{\left(2 m_{1}\left(x^{2}+y^{2}\right)\right)^{1 / 2}}=\frac{\mathbf{c}}{\left(2 m_{1} \mu_{1}^{2}\right)^{1 / 2}},
$$

por lo cual, el lado izquierdo de esta última desigualdad es el potencial clásico más la acción del momento angular, lo cual coincide exactamente con la primer componente de nuestra descomposición de la velocidad, $W_{1}$. Nuevamente tenemos una relación entre las constantes de movimiento; integral de energía total y momento angular con el escape de partículas, ya que la función obtenida en la ecuación (5.9) se encuentra en términos de las distancias mutuas entre partículas, el nivel de energía $h$ en el que nos encontremos y el momento angular, es decir, tenemos la región de Hill generalizada, una herramienta bastante útil para describir la dinámica del problema isósceles en rotación. 
Estudiemos la dinámica de las partículas en la frontera, esto es, consideremos la igualdad

$$
\frac{\mathbf{c}^{2}}{4 m_{1} \mu_{1}^{2}}=U+h
$$

donde al sustituir la función potencial de la ecuación (5.8) obtenemos

$$
\frac{\mathbf{c}^{2}}{4 m_{1} \mu_{1}^{2}}-\frac{1}{\mu_{1}}\left(\frac{m_{1}^{2}}{2}+\frac{2 m_{1} m_{2}}{\sec \varphi}\right)-h=0 .
$$

Multiplicando por $-\mu_{1}^{2}$ la igualdad anterior y nombrando

$$
\frac{m_{1}^{2}}{2}+\frac{2 m_{1} m_{2}}{\sec \varphi}=G(\varphi)
$$

ésta toma la forma de un polinomio cuadrático, el cual denotamos de la siguiente forma

$$
P\left(\mu_{1}\right)=h \mu_{1}^{2}+G(\varphi) \mu_{1}-\frac{\mathbf{c}^{2}}{4 m_{1}}
$$

de aquí la región de Hill generalizada dada por la ecuación (5.9) puede ser escrita como

$$
\frac{\mathbf{c}^{2}}{4 m_{1} \mu_{1}^{2}} \leq \frac{G(\varphi)}{\mu_{1}}+h
$$

con ayuda de la desigualdad (5.10) y el polinomio $P\left(\mu_{1}\right)$, analizamos lo que puede ocurrir con la dinámica de las partículas.

(a) Si $h>0$, se obtiene la siguiente restricción para la dinámica de las partículas

$$
h \mu_{1}^{2}+G(\varphi) \mu_{1}-\frac{\mathbf{c}^{2}}{4 m_{1}} \geq 0
$$

gráficamente este polinomio corresponde a una parábola que abre hacia arriba (véase la figura $5.5(\mathrm{a}))$, lo cual implica que $\mu_{1}$ puede tomar los valores

$$
\mu_{1} \geq \frac{-G(\varphi)+\sqrt{G^{2}(\varphi)+\frac{h \mathbf{c}^{2}}{m_{1}}}}{2 h}, \quad \forall \quad \varphi \in[0, \pi / 2),
$$

la gráfica muestra como las partículas sobre el plano $x-y$ tiene movimiento no acotado, es decir, éstas pueden escapar a infinito, luego de la relación $\mu_{2}=\mu_{1} \tan \varphi$ se obtiene que la partícula de masa $m_{2}$ también puede presentar escape al infinito, entonces cuando el nivel de energía $h$ es positivo, todas las partículas tienen movimiento no acotado.

(b) Si $h=0$, es fácil obtener que $P\left(\mu_{1}\right)$ es una recta (véase la figura $5.5(\mathrm{~b})$ ) y en este caso la distancia de las partículas de masa $m_{1}$ al origen están restringidas a tomar los valores

$$
\mu_{1} \geq \frac{\mathbf{c}^{2}}{4 m_{1} G(\varphi)}, \quad \forall \quad \varphi \in(0, \pi / 2)
$$

es decir, las partículas en el plano $x$-y pueden escapar a infinito. La partícula con masa $m_{2}$ sobre el eje $z$, también puede escapar, puesto que si $\mu_{1} \rightarrow \infty$, de la relación $\mu_{2}=$ $\mu_{1} \tan \varphi$ se obtiene que $\mu_{2} \rightarrow \infty$, esto es, las tres partículas pueden tener movimiento no acotado, cuando el nivel de energía en el que nos encontramos es cero. 
(c) Si el nivel de energía en el que nos encontramos es negativo, $h<0$, la desigualdad de la ecuación (5.10) se puede escribir como

$$
\frac{\mathbf{c}^{2}}{4 m_{1}} \leq G(\varphi) \mu_{1}-|h| \mu_{1}^{2}
$$

de aquí obtenemos

$$
-|h| \mu_{1}^{2}+G(\varphi) \mu_{1}-\frac{\mathbf{c}^{2}}{4 m_{1}} \geq 0
$$

en este caso la gráfica del polinomio $P(\varphi)$ es una parábola que abre hacia abajo (véase la figura $5.5(\mathrm{c}))$, lo cual implica que siempre que $\Delta=G^{2}(\varphi)-\frac{\mathbf{c}^{2}|h|}{m_{1}} \geq 0$ la distancia que pueden tomar las partículas de masa $m_{1}$ al origen, se encuentran en el intervalo cerrado

$$
\left[\frac{-G(\varphi)+\sqrt{\Delta}}{-2|h|}, \frac{-G(\varphi)-\sqrt{\Delta}}{-2|h|}\right], \quad \forall \quad \varphi \in[0, \pi / 2),
$$

es decir, el movimiento de las partículas en el plano $x-y$ es acotado, éstas nunca colisionan entre ellas y tampoco escapan a infinito, entonces de igual forma la distancia al origen de la masa sobre el eje $z$ permanece acotada, esto es, nunca colisionan con las otras dos masas y no escapa a infinito.

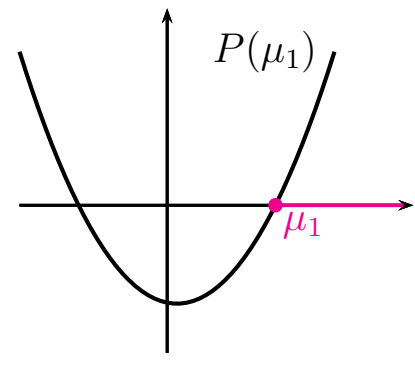

(a) $\mu_{1} \geq \frac{-G(\varphi)+\sqrt{G^{2}(\varphi)+\frac{\mathrm{c}^{2} h}{m_{1}}}}{2 h}$

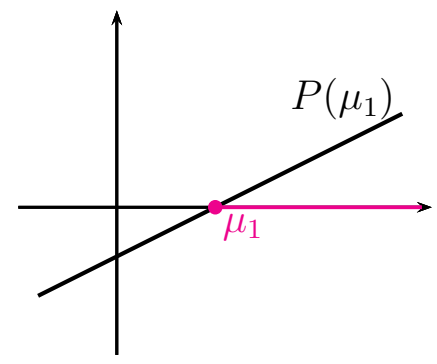

(b) $\mu_{1} \geq \frac{\mathrm{c}^{2}}{4 m_{1} G(\varphi)}$

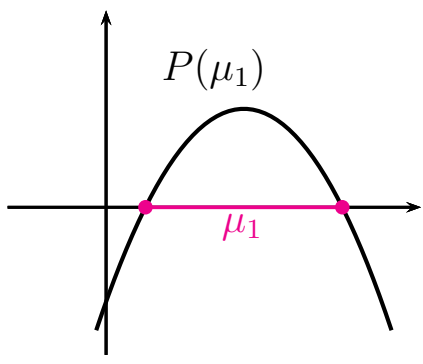

(c) $\mu_{1} \in\left[\frac{-G(\varphi)+\sqrt{\Delta}}{-2|h|}, \frac{-G(\varphi)-\sqrt{\Delta}}{-2|h|}\right]$

Figura 5.5: Gráficas de $P\left(\mu_{1}\right)$ del problema isósceles en rotación.

Para este último caso, es interesante preguntarse sí puede haber escape para la masa $m_{2}$, intuitivamente si el momento angular es grande entonces la masa $m_{2}$ no presenta escape, de lo contrario, si el momento angular es pequeño entonces la masa $m_{2}$ podría escapar. Con el propósito de encontrar para qué casos se puede dar el escape de la partícula sobre el eje $z$, analizamos la función $G(\varphi)$. Para todo $\varphi \in[0, \pi / 2)$ la función $G(\varphi)>0$, la pendiente en cada uno de sus puntos es negativa, es decir,

$$
G^{\prime}(\varphi)=-2 m_{1} m_{2} \operatorname{sen} \varphi<0
$$

tiene un punto crítico en $\varphi=0$, esto es, $G^{\prime}(0)=0$, luego, se tiene que

$$
G^{\prime \prime}(\varphi)=-2 m_{1} m_{2} \cos \varphi
$$


de aquí que $G^{\prime \prime}(0)=-2 m_{1} m_{2}<0$, por lo tanto

$$
G(0)=\frac{m_{1}^{2}}{2}+2 m_{1} m_{2}
$$

es un máximo de la función $G(\varphi)$. Además, si calculamos el límite de $G(\varphi)$ cuando $\varphi \rightarrow \frac{\pi}{2}$ se obtiene

$$
\lim _{\varphi \rightarrow \frac{\pi}{2}} G(\varphi)=\frac{m_{1}^{2}}{2} .
$$

Por el análisis anterior, es posible dibujar la gráfica de la función $G(\varphi)$, la cual se observa en la figura 5.6.

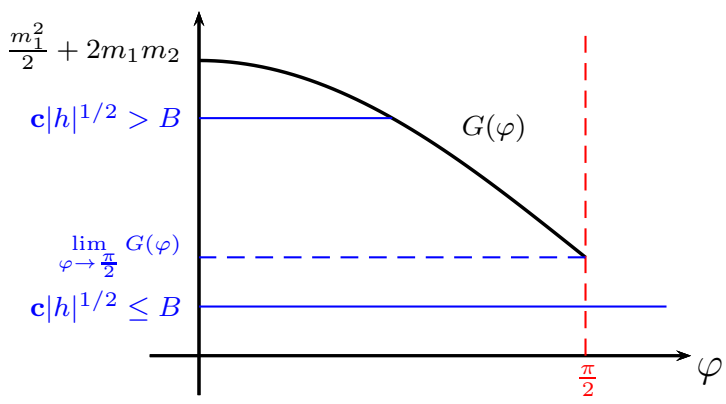

Figura 5.6: Gráfica de $G(\varphi)$.

Ya hemos dicho que el polinomio dado por la ecuación (5.11) tiene raíces reales positivas, por lo cual su discriminante $\Delta$ cumple

$$
\Delta=G^{2}(\varphi)-\frac{\mathbf{c}^{2}|h|}{m_{1}} \geq 0
$$

lo cual implica tener la siguiente desigualdad

$$
\mathbf{c}^{2}|h| \leq m_{1} G^{2}(\varphi), \quad \forall \varphi \in[0, \pi / 2),
$$

cuando $\phi$ va de 0 a $\pi / 2$, el mínimo valor que $\mathbf{c}|h|^{1 / 2}$ puede alcanzar es cuando $\phi \rightarrow \pi / 2$, entonces

$$
\mathbf{c}^{2}|h|=m_{1} \lim _{\varphi \rightarrow \pi / 2} G^{2}(\varphi)=\frac{m_{1}^{5}}{4}
$$

Hemos encontrado el valor de $\mathbf{c}^{2}|h|$ para el cual el escape de la partícula sobre el eje $z$ es posible, haciendo $B=\frac{m_{1}^{5}}{4}$ se obtienen los siguientes dos casos:

- Si $\mathbf{c}^{2}|h| \leq B$ entonces la masa $m_{2}$ escapa a infinito, (véase figura 5.6).

- Si $\mathbf{c}^{2}|h|>B$ entonces la partícula sobre el eje $z$ tiene movimiento acotado como se puede ver en la figura 5.6, lo cual se debe a que el mínimo valor que puede alcanzar $\mathbf{c}|h|^{1 / 2}$ se tiene cuando hacemos tender $\phi \rightarrow \pi / 2$, ya que el ángulo $\varphi$ no puede ser mayor o igual que $\pi / 2$. 
En particular, hemos obtenido las condiciones necesarias para poder tener escapes de la masa $m_{2}$, cuando la energía es negativa. Notemos nuevamente, que el lado izquierdo de las desigualdades de los casos anteriores, corresponde a lo que hemos estudiado como el límite inferior de la medida configuracional, $I U^{2}$, la cual mide cómo cambia la forma de la configuración.

Para concluir con este capítulo, es importante mencionar que como aplicación importante de la descomposición de la velocidad, encontramos una relación entre las constantes de movimiento, integral total y momento angular con el escape de partículas del problema romboidal generalizado en rotación y el problema isósceles en rotación. 


\section{CAPÍTULO 6}

\section{Conclusiones y perspectivas}

\section{Conclusiones}

En este trabajo se realizó un estudio de la descomposición de la velocidad total de un sistema de $N$-partículas moviéndose bajo sus atracciones gravitacionales, la cual denominamos como la descomposición de la velocidad de Saari. Primero se abordaron conceptos y resultados ya conocidos del problema de los $N$-cuerpos (capítulo 1), tales como las integrales primeras, momento angular y momento de inercia. En particular, se estudiaron las regiones de Hill, y se observó que éstas permiten clasificar de manera clara ciertos movimientos en el espacio de configuraciones, es decir, las regiones de Hill clasifican las soluciones en acotadas o no acotadas en términos de la distancia entre las partículas y el nivel de energía en el que nos encontremos. En este trabajo describimos que un cambio configuracional en el problema de los $N$-cuerpos se debe entender como un cambio en la forma (deformación) que no contempla homotecias o rotaciones de ningún tipo. Mediante propiedades de funciones homogéneas, obtuvimos una función homogénea de grado cero, $I U^{2}$, la cual resultó ser invariante ante homotecias y rotaciones, lo cual permitió establecer que esta función homogénea de grado cero mide cómo la forma de la configuración está cambiando.

Se obtuvo la desigualdad de Sundman, la cual conecta los conceptos de energía cinética, momento de inercia y momento angular, de la siguiente manera

$$
\frac{\dot{I}^{2}}{4}+c^{2} \leq I T
$$

Con ayuda de ésta encontramos una restricción de los valores posibles que puede tomar la medida configuracional, $I U^{2}$. Dicha restricción se estudio para valores de energía $h<0$ que resulta ser el caso más interesante, obteniendo que la función que mide el cambio de la 
forma en la configuración de las partículas, está acotada inferiormente por el mínimo $c^{2}|h|$, es decir, para cualquier problema de los $N$-cuerpos y $h<0$ se cumple

$$
c^{2}|h| \leq I U^{2}
$$

Después de esto estudiamos las configuraciones centrales y vimos que la principal aportación de ellas, es que generan las únicas soluciones explícitas del problema de los $N$-cuerpos, las soluciones homográficas, para las cuales existen dos casos límite: las soluciones homotéticas, aquellas que permiten que las partículas cambien el tamaño en la escala preservando al mismo tiempo la configuración, pero sin rotación de ningún tipo; y los equilibrios relativos, en las cuales las distancias entre todos los cuerpos permanecen fijas por lo que las partículas giran sin que haya cambio en la escala. Demostramos que una configuración central es un punto crítico de la función que mide cómo cambia la forma de la configuración.

Posteriormente el estudio se extendió al objetivo fundamental de este trabajo, la descomposición de la velocidad de Saari (capítulo 2), donde se dio el planteamiento geométrico que dio origen a la idea de descomponer ortogonalmente la velocidad total del sistema, después para obtener una representación de la descomposición de la velocidad realizamos proyecciones del vector $\mathbf{v}$ con respecto a un producto interno, el cual además permitió escribir en forma compacta el momento de inercia y las integrales de movimiento, observamos que la $i$-ésima componente del momento angular es la proyección (componente) de $\mathbf{v}$ a lo largo de la linea que contiene al vector $\mathbf{E}_{i} \times \mathbf{r}$ es una constante, fue así como iniciamos la descomposición de la velocidad del sistema obteniendo los vectores, $W_{1}$ : velocidad rotacional, $W_{2}$ : velocidad escalar y $W_{3}$ : velocidad configuracional.

Al estudiar el comportamiento de cada una de ellas obtuvimos:

- La componente de la velocidad, $W_{1}$, está determinada de manera única por el momento angular $c_{i}=\left\langle\mathbf{E}_{i} \times \mathbf{r}, \mathbf{v}\right\rangle$ y la base $\left\{\mathbf{E}_{i} \times \mathbf{r}\right\}_{i=1}^{3}$, de la forma

$$
W_{1}=\sum_{i=1}^{3} s_{i} \frac{\mathbf{E}_{i} \times \mathbf{r}}{\left|\mathbf{E}_{i} \times \mathbf{r}\right|}
$$

donde $s_{i}=\frac{c_{i}}{\left|\mathbf{E}_{i} \times \mathbf{r}\right|}$ está relacionado con la $i$-ésima componente del momento angular. Además el espacio tangente, $S_{\text {rot }}(\mathbf{r})=T_{\mathbf{r}}(\mathcal{M}(\mathbf{r}))$ define un subespacio de $T_{\mathbf{r}}\left(\mathbb{R}^{3}\right)^{N-1}$ el cual sirve como hogar para las componentes del vector de velocidad del sistema que definen la rotación del cuerpo rígido, y su $\operatorname{dim}\left(S_{\text {rot }}(\mathbf{r})\right)=3$, por lo cual, concluimos que $W_{1}$ vive en un subespacio de dimensión tres.

- La velocidad escalar, $W_{2}$, representa el cambio en el tamaño del sistema, esto es, la forma de la configuración nunca cambia solamente su escala, y mostramos que está dada por la razón de cambio en el tamaño de $\mathbf{r}$ en la dirección de un vector unitario,

$$
W_{2}=\left(\frac{d}{d t}|\mathbf{r}|\right) \frac{\mathbf{r}}{|\mathbf{r}|}
$$


Al estudiar el comportamiento de $\frac{d}{d t}|\mathbf{r}|$ pudimos ver que ésta, es la proyección de $\mathbf{v}$ a lo largo del subespacio $S_{\text {scal }}(\mathbf{r})=\left\{t \frac{\mathbf{r}}{|\mathbf{r}|}: t \in \mathbb{R}\right\}$, por lo tanto

$$
W_{\text {scal }}=\left\langle\frac{\mathbf{r}}{|\mathbf{r}|}, \mathbf{v}\right\rangle \frac{\mathbf{r}}{|\mathbf{r}|},
$$

y por lo cual $W_{2}$ siempre vive en un subespacio de dimensión uno. Además, nos dimos cuenta que esta segunda componente de la velocidad, se encuentra relacionada con la razón de cambio con respecto al tiempo del momento de inercia, $I$, de la siguiente manera

$$
W_{2}(\mathbf{r}, I)=\frac{\dot{I}}{2 \sqrt{I}} \frac{\mathbf{r}}{|\mathbf{r}|} .
$$

- Obtuvimos que la velocidad configuracional, es el subespacio final, $S_{\text {conf }}(\mathbf{r})$, que consiste en esas componentes de la velocidad que cambian la forma de la configuración r. Matemáticamente, $S_{\text {conf }}(\mathbf{r})$ es el complemento ortogonal en $T_{\mathbf{r}}\left(\mathbb{R}^{3}\right)^{N-1}$ del espacio generado por $S_{\text {rot }}(\mathbf{r})$ y $S_{\text {scal }}(\mathbf{r})$, y la definimos por la relación

$$
W_{\text {conf }}=\mathbf{v}-\left(W_{1}+W_{2}\right) .
$$

Determinamos que la dimensión del tercer subespacio $S_{\text {conf }}$, en el cual vive el vector $W_{3}$, tiene una dimensión generalmente bastante grande; ya que un recuento de dimensiones nos hizo obtener que la dimensión de este subespacio es

$$
\operatorname{dim}\left(S_{\text {conf }}\right)=3 N-7 .
$$

Vimos que la función homogénea de grado cero, $I U^{2}$, la cual mide el cambio en la forma de la configuración, mide a $W_{3}$, por lo que si $I U^{2}=0$ entonces $W_{3}=0$.

En conclusión, descompusimos la velocidad $\mathbf{v}$ ortogonalmente con respecto a un producto interior $\langle$,$\rangle como \mathbf{v}=W_{1}+W_{2}+W_{3}$. La primer componente, $W_{1}$ es tangente a la subvariedad $\mathcal{M}(\mathbf{r})$, que representa la totalidad de los movimientos de rotación de las partículas vistas como un cuerpo rígido, es decir, $W_{1}$ es la componente de $\mathbf{v}$ que está en el espacio tangente de la subvariedad de rotaciones del cuerpo rígido, $T_{\mathbf{r}}(\mathcal{M}(\mathbf{r}))$. Para encontrar una representación de $W_{\text {rot }}$, proyectamos $\mathbf{v}$ sobre $T_{\mathbf{r}}(\mathcal{M}(\mathbf{r}))$ donde la proyección es con respecto al producto interno $\langle$,$\rangle . La segunda componente, W_{2}$ captura los componentes de la velocidad que están cambiando el tamaño de la configuración y la cual está en la dirección del vector unitario $\frac{\mathbf{r}}{|\mathbf{r}|}$. Dado que este vector es ortogonal a la subvariedad $\mathcal{M}(\mathbf{r})$, es obvio que $W_{1}$ y $W_{2}$ son vectores ortogonales. La última componente $W_{3}$ está dada por $W_{3}=\mathbf{v}-\left(W_{1}+W_{2}\right)$. De acuerdo con la construcción, estos tres vectores son ortogonales entre sí. Además, $W_{3}$ es tangente a $\mathcal{M}(\mathbf{r})$, y ésta es la componente de la velocidad que cambia la forma de la configuración.

Una vez estudiada la descomposición de la velocidad de Saari, el primer problema que nos vino a la mente para obtener la descomposición ortogonal del vector de velocidad del sistema, fue el problema de los 2-cuerpos, obteniendo que dicha descomposición es de la forma: 
$\mathbf{v}=W_{1}+W_{2}$, es decir, la componente de la velocidad que cambia la forma de la configuración es cero, $W_{3}=0$, lo cual indica que las partículas presentan únicamente movimientos rotación como cuerpos rígidos y movimientos homotéticos.

Utilizando la descomposición de la velocidad, dimos una nueva clasificación para problemas de los $N$-cuerpos (capítulo 3), dicha clasificación se basa en la dinámica de las partículas, y no en el número de partículas que se estén considerando. Para iniciar con la clasificación definimos problemas de los $N$-cuerpos cero-dimensional, los cuales están donde $W_{3}=0$, y lo cual nos llevó a tener la siguiente expresión para la descomposición de la velocidad

$$
\mathbf{v}=W_{1}+W_{2},
$$

obteniendo dos importantes casos particulares:

(a) Si $W_{1} \neq 0$ y $W_{2}=0$ entonces tenemos soluciones en equilibrios relativos.

(b) Si $W_{1}=0$ y $W_{2} \neq 0$ entonces las soluciones son homotéticas.

De lo cual concluimos, que si $W_{1} \neq 0$ y $W_{2} \neq 0$ entonces obviamente obtenemos las soluciones homográficas. Justificamos que nuestra definición de problema cero-dimensional, tiene que ver con que las soluciones encontradas por Euler y Lagrange, son ejemplos de soluciones homográficas, y fueron las primeras soluciones del problema de los tres cuerpos, y que en cierto sentido son las órbitas naturales más fáciles de describir. Otro ejemplo fue el problema de los dos cuerpos, el cual vimos en el capítulo dos, que éste también cumple con ser un sistema cero-dimensional, puesto que $W_{3}=0$, implicando que las soluciones mantienen la configuración central de los dos cuerpos durante toda la evolución del sistema.

Con el propósito de continuar con la clasificación de problemas de los $N$-cuerpos, y como una aportación de este trabajo se dio la definición de problemas de los $N$-cuerpos unodimensional, aquí $W_{3}$ debe ser distinto de cero y el centro de masa juega un papel importante cuando dividimos en dos cúmulos el sistema de partículas. Dimos tres ejemplos de sistemas uno-dimensionales: problema romboidal generalizado en rotación, problema isósceles en rotación y el problema colineal de 3-cuerpos en el plano.

Obtuvimos explícitamente las tres componentes de la descomposición de la velocidad de Saari para cada uno de los sistemas uno-dimensional (capítulo 4), para esto, obtuvimos diferentes sistemas coordenados para encontrar una representación de las tres componentes de la descomposición. Encontrar la descomposición del vector de velocidad del problema romboidal generalizado en rotación, la del problema isósceles en rotación y la del problema colineal de 3-cuerpos en el plano, las cuales son otra aportación de este trabajo.

Introdujimos las coordenadas de Fujiwara como nuevas coordenadas para problemas de los $N$-cuerpos en el plano, mostramos que el momento angular en términos de coordenadas de Fujiwara es cero, y que el momento de inercia en términos de las mismas es constante, además pudimos ver que las coordenadas de Fujiwara son independientes tanto del tamaño del sistema como del momento angular, lo cual nos sugirió que la evolución de sistemas de partículas expresado en coordenadas de Fujiwara, describe el cambio en la forma de la configuración dada por las variables originales. Probamos que una solución en el plano es 
homográfica si y sólo si la derivada con respecto al tiempo de las coordenadas de Fujiwara es nula.

Al estudiar la forma de las tres componentes de la velocidad en términos de las coordenadas de Fujiwara, nos dimos cuenta que las coordenadas de Fujiwara están relacionadas con la descomposición de la velocidad de Saari. Como otra aportación de este trabajo aplicamos los resultados obtenidos al problema colineal de 3-cuerpos en el plano, dividiendo el análisis en dos casos: el primero fue considerado movimiento de rotación en las partículas y en el segundo supusimos que las partículas en configuración colineal permanecían sin movimiento de rotación. Observamos que los resultados que se obtuvieron en ambos casos contenían términos semejantes a los encontrados con la descomposición de la velocidad de Saari para el problema colineal de 3-cuerpos en el plano (capítulo 3), lo cual nos hizo pensar que la descomposición de la velocidad en términos de las coordenadas de Fujiwara, permite obtener resultados similares a los que se pudieran obtener en problemas de los $N$-cuerpos en el plano, con la descomposición de la velocidad de Saari original.

Nos dimos cuenta que aplicar la descomposición de la velocidad de Saari a problemas de los $N$-cuerpos permitía estudiar su dinámica (capítulo 5), ya que utilizando la integral de energía total junto con la descomposición de la velocidad, obtuvimos la generalización de las regiones de Hill,

$$
2(U+h)-\lambda_{1}^{2} \geq 0,
$$

con éstas trabajamos la dinámica de las partículas en la frontera del problema romboidal generalizado en rotación y del problema isósceles en rotación, obteniendo en qué casos los movimientos de las partículas presentan escapes; en particular estudiamos para qué valores de $\mathbf{c}|h|^{1 / 2}$ las partículas escapan.

Las aportaciones de este trabajo se pueden resumir en tres: la introducción de la descomposición de la velocidad total de Saari a problemas de los $N$-cuerpos, una nueva clasificación de problemas de los $N$-cuerpos en términos de la descomposición de la velocidad de Saari, y la obtención de las regiones de Hill generalizas, las cuales permiten obtener en que casos los movimientos de las partículas presentan escapes.

\section{Perspectivas}

Finalmente, mencionaremos que el trabajo realizado en esta tesis da inicio a estudios más profundos de la descomposición de la velocidad de Saari. Las posibilidades de trabajo futuro son buenas, por ejemplo:

- Continuar clasificando problemas de los $N$-cuerpos en términos de la descomposición de la velocidad, es decir, poder definir sistemas dos-dimensional, tres-dimensional, etcétera, lo cual es un reto.

- Estudiar lo que sucede con la dinámica de las partículas al tomar la desigualdad

$$
2(U+h)-\lambda_{2}^{2} \geq 0
$$


de la cual tenemos una función en términos de las distancias mutuas entre las partículas, el nivel de energía en el que nos encontremos y la razón de cambio en el tamaño del sistema $(\dot{I})$.

- Otra posibilidad es estudiar la desigualdad

$$
2(U+h)-\left(\lambda_{1}^{2}+\lambda_{2}^{2}\right) \geq 0
$$

para conocer los movimiento que se producen, y creemos que ésta proporcionara información muy valiosa sobre la dinámica de las partículas, ya que con esta desigualdad tenemos una función en términos de las distancias mutuas entre las partículas, el nivel de energía en el que nos encontremos, el momento angular y la razón de cambio en el tamaño del sistema.

- Por último, también nos gustaría poder hablar con toda claridad de sistemas $N$ dimensional.

En conclusión, el estudio de la descomposición de la velocidad de Saari ofrece aún muchas preguntas, y sobre todo, en el empleo de técnicas que pueden llevar a entender mejor la dinámica tan complicada de los problemas clásicos, por esto mismo el desarrollo de este trabajo puede pensarse como una buena introducción a las características de la descomposición de la velocidad del sistema y dejar en firme los conceptos que son necesarios para estudios posteriores que permitan continuar clasificando problemas de los $N$-cuerpos en términos de las componentes del vector de velocidad del sistema. 
[1] Boccaletti, D., and Pucacco, G. Theory of orbits Volume 1: Integrable systems and nonperturbative methods, Third Edition, Springer-Verlag Berlin Heidelberg, Germany 1996.

[2] Diacu, F., Fujiwara, T., Pérez-Chavela, E., and Santopetre, M. Saari's homographics conjeture of the three-body problem, Transactions of the American Mathematical Society Volume (360), Number 12, 6447-6473, (2008).

[3] Franco, L. El problema restringido de los tres cuerpos en S1, Tesis de maestría, UAMIztapalapa, (2006).

[4] Lacomba-Zamora, E. Movimientos con singularidades o escapes en Mecánica Celeste, Aportaciones internas, Departamento de Matemáticas, UAM-Iztapalapa, No. 27, (1984).

[5] McGehee, R. Triple collision in the collinear three body problem, Inventiones Mathematicae 27, 191-227, (1974).

[6] Meyer, K., Hall, G., and Offin, D. Introduction to hamiltonian dynamical systems and the n-body problem, Second Edition, Springer-Verlag New York, USA 2009.

[7] Moser, J. Stable and random motions in dynamical systems, Princeton University Press 1973.

[8] Pérez-Chavela, E. Different kinds of potentials in celestial mechanics, Dynamics of Continuous, Discrete and Impulsive Systems 5, 545-560, (1999).

[9] Saari, D. Singularities and collisions of Newtonian gravitational systems, Archive for Rational Mechanics and Analysis 49, 311-320, (1973).

[10] Saari, D.The n-body problem of celestial mechanics, Celestial Mechanics 14, 11-17, (1976). 
[11] Saari, D. From rotations and inclinations to zero configurational velocity surfaces I; a natural rotationg coordinate system, Celestial Mechanics and Dynamical Astronomy 33, 299-318, (1985).

[12] Saari, D. From rotations and inclinations to zero configurational velocity surfaces II; the best possible configurational velocity surfaces, Celestial Mechanics and Dynamical Astronomy 40 ,197-223, (1987).

[13] Saari, D. Symmetry in n-Particle Systems, Contemporary Mathematics, Volume 81, 23-42, (1988).

[14] Saari, D. Collisions, Rings, and Other Newtonian N-Body Problems, American Mathematical Society, Regional Conference Series in Mathematics, No. 104, Providence, RI, 2005.

[15] Saari, D. Central configurations - A problem for the twenty-first century, Expeditions in Mathematics, MAA SPECTRUM, 283-295, (2011).

[16] Simó, C., and Martínez, R. Qualitative study of the planar isosceles three-body problem, Celestial Mechanics 41, 179-251, (1988).

[17] Wintner, A. The analytic foundations of Celestial Mechanics, Princeton University Press, Princeton NJ 1941. 